\title{
Precision physics of simple atoms: QED tests, nuclear structure and fundamental constants
}

\author{
Savely G. Karshenboim \\ D. I. Mendeleev Institute for Metrology, 190005 St. Petersburg, Russia \\ Max-Planck-Institut für Quantenoptik, 85748 Garching, Germany
}

\begin{abstract}
Quantum electrodynamics is the first successful and still the most successful quantum field theory. Simple atoms, being essentially QED systems, allow highly accurate theoretical predictions. Because of their simple spectra, such atoms have been also efficiently studied experimentally frequently offering the most precisely measured quantities. Our review is devoted to comparison of theory and experiment in the field of precision physics of light simple atoms. In particular, we consider the Lamb shift in the hydrogen atom, the hyperfine structure in hydrogen, deuterium, helium-3 ion, muonium and positronium, as well as a number of other transitions in positronium. Additionally to a spectrum of unperturbed atoms, we consider annihilation decay of positronium and the $g$ factor of bound particles in various two-body atoms. Special attention is paid to the uncertainty of the QED calculations due to the uncalculated higher-order corrections and effects of the nuclear structure. We also discuss applications of simple atoms to determination of several fundamental constants.
\end{abstract}

Key words: Simple atoms, Precision measurements, Bound states, Quantum electrodynamics (QED), Hydrogen-like atoms, Fundamental constants PACS: $12.20 . \mathrm{Fv}, 12.20 . \mathrm{Ds}, 31.30 . \mathrm{Jv}, 06.02 . \mathrm{Jr}, 31.30 . \mathrm{Gs}, 36.10 . \mathrm{Dr}, 13.40 . \mathrm{Em}$, 13.40.Gp, 27.10.+h

Email address: sek@mpq.mpg.de (Savely G. Karshenboim). 


\section{Contents}

$1 \quad$ Introduction 4

$2 \quad$ Spectrum of simple atoms and main QED phenomena 13

3 Optical measurements in hydrogen atom and determination of the Rvdberg constant 21

$4 \quad$ The Lamb shift in the hvdrogen atom 24

$5 \quad$ Lamb shift and effects of the proton charge distribution 34

$6 \quad$ Hvperfine splitting in light hvdrogen-like atoms and the nuclear structure 39

$7 \quad$ Hvperfine structure. free of the nuclear effects: comparison of the HFS intervals for the $1 s$ and $2 s$ st

$8 \quad$ Hvperfine splitting in the ground state of muonium 49

$9 \quad$ Spectrum and annihilation of positronium and recoil effects 52

10 The $q$ factor of an electron and a nucleus in a light hvdrogen-like atom 60

$\begin{array}{ll}10.1 \text { General consideration } & 60\end{array}$

10.2 Hvdrogen and its isotopes: the isotopic effects for the $q$ factor of a bound electron 62

10.3 Hvdrogen and deuterium: determination of the proton and deuteron magnetic moments 64

10.4 The $g$ factors of an electron and a muon in muonium and the determination of the muon mass

66

11 The $g$ factor of a bound electron in a hvdrogen-like ion with a spinless nucleus and a determination

12 A determination of the fine structure constant $\alpha$ by means of QED and atomic physics 72

13 Higher-order logarithmic corrections and the uncertainty of the bound-state QED calculations

75

13.1 Hvperfine structure in light hvdrogen-like-atoms 75

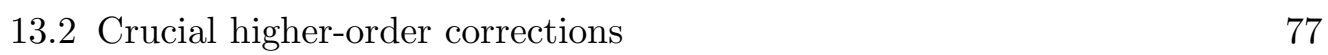

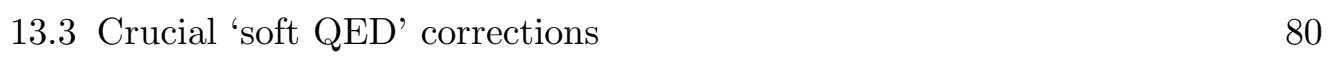

\begin{tabular}{|l|l}
13.4 Crucial 'hard QED' corrections & 81
\end{tabular}

14 What is next?

$\begin{array}{lll}15 & \text { Summary } & 86\end{array}$

\begin{tabular}{lll}
\hline A Notations & 89
\end{tabular} 
B Extract from the list of the recommended fundamental constants (CODATA. 2002) 89

$\begin{array}{ll}\text { References } & 90\end{array}$ 


\section{Introduction}

Several atoms play basic roles in modern physics and, in fact, very different roles. A unit of time, the second, is defined via the hyperfine interval in the cesium atom, while the atomic mass unit and the Avogadro number are defined via the mass of a carbon atom. These two atoms are significant for our system of units, SI. In addition, there are some favorite atomic systems where the basic laws of Nature find their expression in the most transparent way. These simple atoms, most of which consist of two bound particles, have been crucial for our understanding of key moments of modern physics and their study is still of essential interest and importance.

The simplicity and harmony of the theory of bound systems have been tempting and challenging for a while. Johannes Kepler believed the Solar planetary system to be governed by a harmony of discrete numbers via geometry, trying with the so-called Platonic or Regular solids. He failed to verify that kind of the harmony and discovered instead some regularities in the planetary orbital motion known now as Kepler's laws. His discovery became a milestone in the development of theory of gravitation and mechanics and eventually led to the establishment of new mechanics (classical mechanics).

Three centuries after the Kepler's time, a planetary model was suggested for atoms. Meantime certain regularities in the spectrum of the hydrogen atom were discovered. Those regularities, like the Kepler's laws, led once again to the establishment of new mechanics (quantum mechanics) and simultaneously realized the Kepler's dream of the harmony of numbers governing the orbital motion.

By now we know that a quantity describing a classical object can be of an arbitrary value while in the quantum case only discrete values are possible for some quantities. And this is how certain integer numbers enter the basic equations of modern physics.

Working on a new mechanics, a new dynamics or a new model, one used to try first to apply it to some 'simple' objects. The simplest object is a free particle. However, only a limited number of properties of a free particle can be derived $a b$ initio and studied with a high accuracy. Study of simple atoms opens a broad field for possible effects and applications. A two-body atomic system such as the hydrogen atom is a natural object to verify a new model or to test a new approach. Studies of the properties of the hydrogen atom have already served to establish the so-called 'old quantum mechanics' (the Bohr theory), modern nonrelativistic quantum mechanics, relativistic quantum mechanics (based on the Dirac equation) and quantum electrodynamics (QED), which was the first successful quantum field theory. Perhaps, we should even say that 
QED is the only quantum field theory which is successful for a really broad range of problems from atomic spectra to scattering, from low energy, related to microwave radiation, to high energy phenomena with hard annihilation and bremsstrahlung, from nano- to giga- electronvolt.

Figure 1 shows several crucial contributions to hydrogen energy levels. We note here that one of reasons for choosing a non-relativistic equation by Schrödinger over a relativistic Klein-Gordon-Fock equation was an incorrect description by the latter of the fine structure effects in the latter. Another remark on importance of the hydrogen atom for QED is that the anomalous magnetic moment of an electron was first discovered by Rabi and his colleagues [1] as an anomaly in the hyperfine structure of hydrogen. Immediately that was interpreted as a possible anomaly related to a free electron and only afterwards was that confirmed by a direct experiment. A historic overview of the 'contribution' of the hydrogen atom to modern physics can be found in [2].

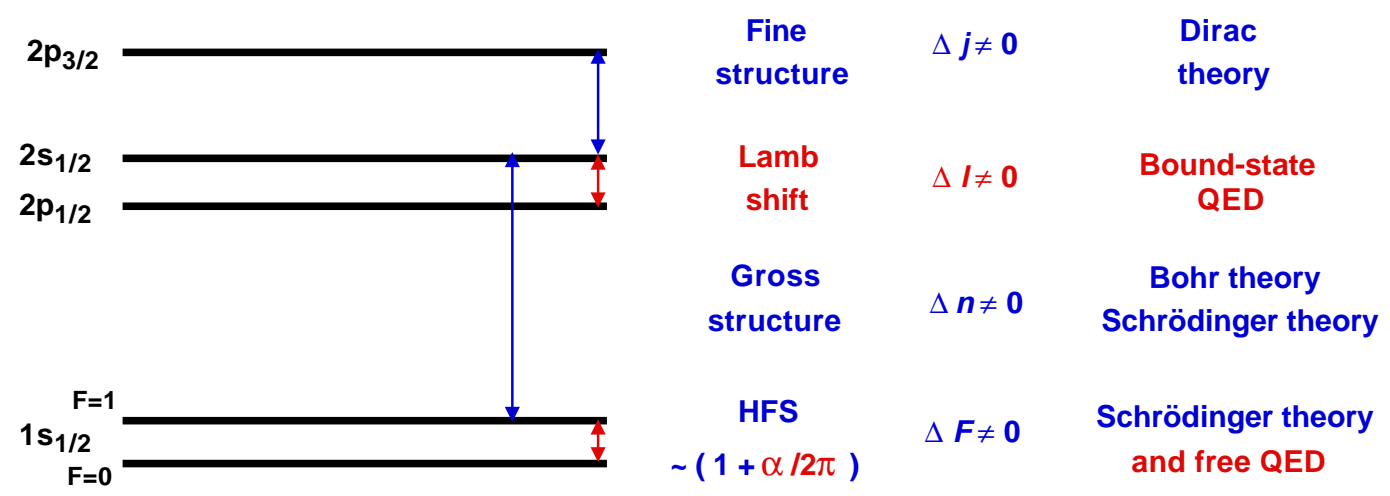

Fig. 1. Some low-lying levels in the hydrogen atom (not to scale). The hydrogen $n l_{j}(F)$ levels are labelled by the values of the principal quantum number $n$, orbital moment $l$, electron angular momentum $j$ and atomic angular momentum $\mathbf{F}=\mathbf{j}+\mathbf{I}$, where $I$ is the nuclear spin. The gross structure $(\Delta n \neq 0)$ is well explained by the Bohr theory (so-called 'old quantum theory') and Schrödinger theory which also predicts the hyperfine structure $(\Delta F \neq 0)$. The fine structure $(\Delta j \neq 0)$ is explained by the Dirac theory while the Lamb shift $(\Delta l \neq 0)$ is a result of bound state QED effects. Meanwhile effects of quantum electrodynamics for free particles are responsible for the $\alpha / 2 \pi$ anomaly in the hyperfine structure.

One can expect that the simplest atoms are the easiest for a theoretical prediction. That is true only in part. A simple atom usually possesses a simple spectrum which has relatively simple and transparent properties. The atomic energy levels are often perturbed during measurements by various external factors such as a residual magnetic or electric field. Because of the simplicity of the spectra, there is a good chance to understand the influence of those external factors on simple atoms. As examples of the highest-precision experiments on simple atoms, we remind here that the hyperfine interval in the ground state of hydrogen was the most accurately measured physical quantity for a few decades and today the $1 s-2 s$ interval in the hydrogen atom is among 
the most precisely measured values.

Decade after decade, theorists and experimentalists investigated simple atoms. As a result their theory is the most advanced atomic theory and it has to compete with very sophisticated experiments. The theory of simple atoms goes now far beyond non-relativistic quantum mechanics with the reduced mass of an electron. One has also to take into account relativistic effects, recoil effects, quantum electrodynamics, effects of the nuclear spin and nuclear structure.

In an early time of modern physics a list of simple atoms consisted of hydrogen only and later deuterium, neutral helium and helium ion were added. Now the list is much longer and quite impressive. It also contains tritium, hydrogenlike ions of almost all elements (and other few-electron ions). Artificial twobody atoms are the easiest to produce and the list also includes muonium and positronium, muonic atoms, pionic, kaonic and antiprotonic atoms, exotic bound systems of two unstable particles (such as pionium and $\pi \mu$-atoms) and antihydrogen.

Often accuracies of theory and experiment are not compatible. However, there is a broad range of effects, for which theory and experiment approach the same high level of accuracy. The study of such effects forms a field called precision tests of bound state $Q E D$, which is reviewed in part here.

A number of sources have contributed to uncertainty of such tests, and the current accuracy of QED calculations for free particles and two-body atoms is not a limiting factor for QED tests. The accuracy of the tests is limited by one of the three other sources:

- an experimental uncertainty;

- an inaccuracy of taking into account effects of the strong interactions;

- an uncertainty due to a determination of the fundamental constants.

The latter enters consideration because theory is not in a position to give itself any quantitative predictions. It provides us with some expressions containing values of certain fundamental constants, such as the Rydberg constant $R_{\infty}$, the fine structure constant $\alpha$, the electron-to-proton mass ratio etc. To make a prediction, one needs first to determine the fundamental constants by extracting their values from some other experiments. Thus, theory serves as a bridge between different experiments. That makes the determination of fundamental physical constants to be another important part of precision physics of simple atoms.

The contemporary situation with the QED uncertainty being below the total uncertainty of any QED test is a result of significant theoretical progress for the two last decades. Twenty or thirty years ago the QED uncertainty was often the dominant one. 
The precision tests of $Q E D$ form a multidisciplinary field involving atomic, nuclear and particle physics, laser spectroscopy, frequency metrology, accelerator physics, advanced quantum mechanics, quantum field theory etc. Those tests are rather not to test QED itself, but to check the overall consistency of the results and methods from different fields and in part to search for possible new physics beyond the Standard Model. In doing more and more advanced QED calculations, we also need to verify our approaches to deal with infrared and ultraviolet divergences, renormalization and bound state problem for fewbody atoms in the case of high order terms of perturbation theory.

As already mentioned, the simplest object to test any theory is a free particle. A study with free leptons provides us with a possibility of testing the QED Lagrangian. The most accurate data for a free particle are related to the anomalous magnetic moments of an electron and a muon. In the former case the limitation of the accuracy of a comparison of theory versus experiment is due to a determination of the fine structure constant $\alpha$ while the latter QED test is limited by effects of strong interactions entering via hadronic intermediate states for the vacuum polarization effects and by the experimental accuracy. The QED theory of the anomalous magnetic moment is quite advanced including many hundreds of multiloop diagrams (up to the four-loop level for the electron and five-loop level for the muon). That differs from a so called bound state $Q E D$, a quantum electrodynamics theory of simple atoms, which deals mainly with relatively few one-loop and two-loop diagrams, but those are not for free particles, but for the particles bound by the Coulomb field. Three-loop contributions are rather below the uncertainty of most of the bound QED calculations and if necessary can be calculated neglecting binding effects.

These two QED theories, the free QED and the bound state QED, are very different in their approaches, problems and applications and it is worth to consider their tests separately. The bound state problem makes all calculations more complicated. Intending to perform a calculation within the free QED, one can in principle find needed basic expressions in a number of textbooks. On the contrary, the bound state QED is not a well-established theory and there are no published common prescriptions for the relativistic quantum bound problem. It involves different effective approaches to solve the two-body bound problem.

Precision tests of the bound state QED offer a number of different options:

- The approaches for the bound problem can be essentially checked with the low- $Z$ two-body atomic systems like hydrogen and deuterium, neutral helium and helium ions, muonium, positronium, etc. At low value of the nuclear charge $Z$ the binding energy is of order of $(Z \alpha)^{2} m c^{2}$ and it is essentially smaller than the energy related to the rest mass $m c^{2}$ of the orbiting parti- 
cle. That is a regime of a weak coupling in contrast to the high- $Z$ physics. The weak coupling allows efficient use of a perturbation expansion over the Coulomb strength $Z \alpha$. Many contributions of higher-order in $Z \alpha$ are calculated diagrammatically and thus are closely related to other QED diagrams (e.g., for scattering theory).

- Studies of high- $Z$ ions are related to a strong coupling regime, however, it is unlikely to provide us with more information on bound state QED because of substantial contributions due to the nuclear structure. Such an investigation is rather useful for testing different nuclear models. However, in some particular cases, atomic systems with not too high $Z$ can give some important information on higher order terms of the QED $Z \alpha$ expansion. Importance of particular atomic systems also depends on the energy interval under study. For instance, the hyperfine structure (HFS) interval depends more strongly on the nuclear-structure effects than the Lamb shift, and the related calculations involve more details of the nuclear structure. The accuracy of the nuclear-finite-size contribution is often claimed to be very high, however, their uncertainty is customarily not well verified. It is often estimated from a variation of the result for an energy shift after application of certain models of the charge distribution while the charge radius is fixed. However, to claim such a calculation as an ab initio result, one has to reconsider first the original scattering and spectroscopy data on the nuclear form factor and related nuclear theory and to check how strongly they constrain the shape of the distribution in general and its parameters and afterwards to question how much the nuclear-size correction varies within allowed shapes and parameters. That may be done only on a nucleus-bynucleus basis. Lack of such a consideration in study of high- $Z$ study reduces importance of the bound-state QED calculations, which are in such a case one more confirmation of applicability of the phenomenological model of the charge distribution.

- Studies of few-electron atoms involve electron-electron interactions. In the case of high $Z$ ions, the electron-electron interaction is proportional to $\alpha$, while the interaction between an electron and the nucleus is proportional to $Z \alpha$. If the ion is highly charged with only few electrons remaining, the electron-electron interaction can be treated as a perturbation. As a result, at high $Z$ the electron must be treated relativistically, i.e., no expansion over $Z \alpha$ can be used, however, the treatment of the electron-electron interaction as a perturbation leads to the $1 / Z$ expansion. In the case of light atoms, electrons are essentially nonrelativistic but the electron-electron interaction is compatible with the nucleus-electron interaction. The few-electron atoms (like, e.g., the neutral helium atom or heavy lithium-like ions) is the only case when the uncertainty of the QED calculations used to be a limiting factor for a comparison of theory versus experiment.

- There are some other two-body atoms under investigation. They contain a muon or a hadron as an orbiting particle. The orbit in a muonic atom is much closer to the nucleus than in the case of a conventional atom with an orbiting 
electron, and the muonic energy levels are much more sensitive to the nuclear structure. In the case of a hadronic atom, the orbit lies even lower than in a muonic atom, while the interaction of the orbiting particle and the nucleus is dominated by the strong interaction, in contrast to the electromagnetic interaction in conventional (i.e., electronic) and muonic atoms. Different exotic or muonic atoms offer a unique opportunity to study certain particle properties by spectroscopic means with high precision.

Frequently high- $Z$ spectroscopy is quoted as a QED test at a strong field. However, that is not exactly the case. A value of macroscopic meaning, such as the electric field strength $\mathbf{E}$, has not much sense inside an atom. Other details are more important. For example, the strength of the field can be characterized by the average potential energy

$$
\langle U\rangle \sim \frac{(Z \alpha)^{2} m c^{2}}{n^{2}}
$$

which increases with the nuclear charge $Z$ and the mass of the orbiting particle $m$ and decreases with the principal quantum number $n$. The strongest field is related to high- $Z$ atoms with an orbiting particle, heavier than electron, at the ground state. Muonic atoms have been studied for a while and with $m_{\mu} \simeq 207 m_{e}$ they offer a test at a field stronger than in electronic atoms and at shorter distances

$$
\langle r\rangle \sim \frac{\hbar}{Z \alpha m c} n^{2}
$$

The distance $\langle r\rangle$ (or a related characteristic value of the momentum transfer $\langle q\rangle \sim \hbar /\langle r\rangle)$ is another important characteristic of the electric field inside an atom.

We also note that a characteristic value of the potential $\langle U\rangle \sim Z \alpha /\langle r\rangle$, the distance $\langle r\rangle$ and the strength of the field $|\mathbf{E}| \sim\langle U\rangle /\langle r\rangle$ strongly depends on a particular quantity under study. What we present above in Eqs. (1) and (2) is related to the leading contributions to atomic energy levels. Higher order corrections, such as QED corrections, may involve various short-distance effects with characteristic distance $\langle r\rangle \sim \hbar / m c$ and momentum transfer of about $m c$. They correspond to higher field than long-distance effects.

The case of strong fields at short distances in which a characteristic momentum transfer is higher than $2 m_{e} c$ leads to an enhancement of polarization effects of the electron-positron vacuum. That makes the physics of muonic atoms quite different from that of conventional atoms. High- $Z$ ions offer another opportunity - a strong coupling regime with the binding energy comparable to $m c^{2}$ which implies a completely relativistic consideration. The strong-coupling 
regime is very different from perturbative weak-coupling one. The calculations are more complicated. They may be of interest for study of methods to be applied in the case of strong interactions. Some of the high- $Z$ results are important also for high-accuracy low- $Z$ physics. However, one has to remember that the strong coupling is rather not a problem of QED, but a problem of its application. In this sense, the stronger the coupling, the less 'simple' the system. To study pure QED problems in more detail we should prefer a weak-coupling situation.

In muonic atoms the coupling constant and other parameters can take quite different values. While for $Z=1,2$ the states of interest are $n=1,2$, the principal quantum number $n$ for medium $Z$ may be higher than 1 or 2 , which used to be common for the high- $Z$ experiments with conventional atoms.

However, in both cases (muonic/exotic atoms and high- $Z$ ions), understanding the nuclear properties is needed for any theoretical predictions. For instance, let us look at studies of the $1 s$ Lamb shift in hydrogen-like uranium. Recently, the experimental result was improved [3]. The experimental uncertainty allows to check one-loop radiative corrections, but not two-loop effects calculated few years ago [4]. Those two-loop corrections are an object of intensive theoretical study and are of great interest (see Sects. 5, 7 and 13). The finite-nuclear-size uncertainty for the Lamb shift in $\mathrm{U}^{91+}$ is estimated at a level approximately tenfold below the experimental uncertainty. However, the uncertainty of the result was obtained [5] (see also [6,7]) by comparison of two distributions of the nuclear charge, which were the homogenous spherical distribution and the Fermi distribution. The value of the mean square radius was fixed as $\left\langle r^{2}\right\rangle^{1 / 2}=5.8604(23) \mathrm{fm}$ for both distributions. However, this result was obtained in Ref. [8] from muonic uranium spectroscopy suggesting a modified Fermi distribution, which is different from both distributions applied in [6]. It was stated [8] that the uncertainty presented there was of pure statistical nature, while the model-dependence had not been studied and related systematic error was not included. Apparently, the characteristic atomic momentum in muonic uranium is much higher than in the conventional hydrogen-like uranium ion and muonic spectra are substantially more sensitive to the nuclear-shape effects than conventional ones. If one expects that a comparison of the homogenous spherical distribution and the Fermi distribution leads to a plausible estimation of the finite-nuclear-size effects (which should be verified), that should be applied first to muonic atoms to learn the systematic error of the mean square radius. Then, with a value of the radius related to each distribution, one should calculate the energy levels. That should substantially increase uncertainty. This example shows that how fragile QED tests with high- $Z$ ions can be and how much work should be additionally done to develop them.

A purpose of this paper is to give a brief review on precision physics of simple 
atoms related to the accurate tests of quantum electrodynamics for bound states and the determination of fundamental constants. Because of that, we focus our considerations mainly on light hydrogen-like atoms (hydrogen, deuterium, helium ion, muonium, positronium) and some medium $Z$ ions, where the nuclear structure and hadronic effects are not too significant and allow a crucial test of advanced QED theory with high order contributions.

We distinguish here QED and bound state QED, which example is an application of QED to the simplest atoms. Studying less simple atoms we deal not with just bound state QED, but with its realization for not-too-simple atoms. The additional problem may be related to strong field, strong coupling, crucial effects due to the nuclear structure, electron-electron interaction in few-electron atoms etc. Definitely, a number of investigations of less simple atoms are of interest. However, dealing with them is to go beyond the simplest bound state QED.

We note, that the light hydrogen-like atoms are the most attractive from a theoretical point of view. They involve neither electron-electron interactions, nor strong-coupling effects or so. We consider in the next section, what is the most favorite choice for experimental accuracy options in testing bound state QED for hydrogen-like atoms. That is related to the light atoms. They are also favorite in principle for theoretical accuracy, being the simplest atomic systems. That does not mean that study of other atoms are out of interest. First of all, what is important is not just an atomic system, but a certain transition there. As one can see in this review, certain transitions, or combinations of certain quantities, related to different transitions, may offer various theoretical or experimental advantages.

Because of the simplicity of simple atoms and multidisciplinary nature of the precision tests of the bound state $Q E D$ we have tried to review the results as simply and briefly as possible in order to make the paper readable by nonexperts. Detailed reference information on crucial theoretical contributions is collected in tables.

While considering QED, there is always the problem of selecting units. From a theoretical point of view one should like to simplify equations by applying relativistic units in which $\hbar=c=1$, or using the atomic units. Meanwhile, from an experimental point of view the equations should be expressed in units convenient for measurements. In our paper we choose a compromise. All results are presented in the units of the SI. However, we present, when possible, most of the results using natural constants as a kind of units. For example, to define the so-called Fermi energy which is a result of the non-relativistic interactions of the electron and nuclear magnetic moments, we write for hydrogen

$$
E_{F}=-\frac{8 \pi \alpha}{3} \frac{\hbar^{3}}{m_{e}^{2} c} \frac{1}{1+a_{e}} \frac{\left\langle\boldsymbol{\mu}_{e} \cdot \boldsymbol{\mu}_{p}\right\rangle}{\mu_{B}^{2}}\left|\Psi_{1 s}(\mathbf{r}=0)\right|^{2}
$$


and thus the proton and electron magnetic moments are explicitly expressed in units of the Bohr magneton. The other factors do not directly contain electron charge $e$, but only the fine structure constant $\alpha$, which does not depend on a choice of 'macroscopic' units (in which it may be defined as $\alpha=e^{2} / \hbar c$, $\alpha=e^{2} / 4 \pi \hbar c, \alpha=e^{2} / 4 \pi \epsilon_{0} \hbar c$ depending on the definition of the unit for the electric charge). Still, here we make an exception for numerical values of atomic energy, which are always expressed in frequency units, i.e., in terms of $E / h$. This is because it is widely preferred to write equations for energy, while the actually measured quantities are the transition frequencies, spectroscopic linewidths and decay rates. The most frequently used notations are explained in Appendix A.

More details on physics of hydrogen-like atoms can be found in:

- various basic questions in books $[9,10]$;

- an overall review with an extended comparison of theory and experiments related to sixties and early seventies in [11];

- minireviews on particular questions in $[10,12,13,14]$;

- review on theory of light hydrogen-like atoms in $[15,16]$;

- original results presented at Hydrogen Atom conferences and on International conferences of Precision Physics of Simple Atomic Systems (PSAS) in $[17,13,14,18,19]$.

The books [13,14] published in series Lecture Notes in Physics, volumes 570 and 627, are also available on-line. The recent PSAS conference on simple atoms took place in early August 2004 in Brazil as a satellite meeting to the International Conference on Atomic Physics (ICAP). The coming PSAS meeting is scheduled for June 2006 in Venice.

A few problems related to our paper are not presented here in detail.

- We consider the fundamental constants only in connection to simple atoms and QED. More detail on fundamental constants can be found in [20,21,22,23,24].

- Heavy few-electron ions, which are of a great interest for study of application of bound state QED to strong-coupling and few-electron systems, are reviewed in, e.g., [6,25,26].

- Recent progress with exotic and muonic atoms is presented in detail in $[27,28,29,30]$. Study of such atoms are not of a big interest because of QED, on contrary, they deliver us a crucial information on other part of physics, namely, particle and nuclear physics.

Most of this review was ready before new results on the fundamental constants [23] became available and through out the paper we compare the QED-related results on the fundamental constants with a previous set of the recommended constants [21]. We also note that a substantial part of QED results under review appeared between the publications of two recommended sets (their 
deadlines for collecting the input data were 1998 and 2002) and most of recent results have been accommodated in [23].

We start our review with an introductory discussion of spectra of simple atoms and related basic QED phenomena and next consider QED tests with hydrogen (the Lamb shift and hyperfine structure) and other light atoms. We discuss studies of pure leptonic atoms such as muonium and positronium. In addition to spectra we consider the magnetic moments of bound particles in various two-body atomic systems. The concluding sections of the paper are devoted to fundamental constants and problems of the bound state QED.

\section{Spectrum of simple atoms and main QED phenomena}

Let us discuss the spectrum of simple two-body atoms in more detail. The gross structure of atomic levels in a hydrogen-like atom comes from the Schrödinger equation with the Coulomb potential and the result is well known:

$$
E_{n}=-\frac{(Z \alpha)^{2} m_{R} c^{2}}{2 n^{2}}
$$

where $Z$ is the nuclear charge in units of the proton charge, $m_{R}$ is the reduced mass of the atomic orbiting particle (mostly, an electron)

$$
m_{R}=\frac{M m}{M+m}
$$

Here, $m$ and $M$ are masses of the orbiting particle and the nucleus.

There are a number of various corrections:

- relativistic corrections (one can find them from the Dirac equation);

- hyperfine structure (due to the nuclear magnetic moment);

- recoil corrections;

- radiative (QED) corrections;

- nuclear-structure corrections.

A structure of levels with the same value of the principal quantum number $n$ is a kind of signature of any atomic system. For most of the precision applications the substructure of interest is related to $n=2$. The corrections decrease with a value of the principal quantum number as $n^{-3}$ or faster. The only exception is the Uehling correction for muonic and exotic atoms which scales as $n^{-2}$ for medium and high $Z$. 


\begin{tabular}{|c|c|c|c|}
\hline Contribution & $\begin{array}{l}\text { Hydrogen-like } \\
\text { electronic atom }\end{array}$ & Positronium & $\begin{array}{l}\text { Hydrogen-like } \\
\text { muonic atom }\end{array}$ \\
\hline $\begin{array}{l}\text { Schrödinger contributions } \\
\text { - with } M=\infty \\
\text { - with } m_{R} \text { (correction) } \\
\text { Relativistic corrections } \\
\text { - Dirac equation } \\
\text { - Two-body effects } \\
\text { Quantum electrodynamics } \\
\text { - Self energy } \\
\text { - Radiative width } \\
\text { - Vacuum polarization } \\
\text { - Annihilation } \\
\text { - virtual } \\
\text { - real } \\
\text { Nuclear effects } \\
\text { - Magnetic moment (HFS) } \\
\text { - Charge distribution }\end{array}$ & $\begin{array}{c}1 \\
m / M \\
(Z \alpha)^{2} \\
(Z \alpha)^{2} m / M \\
\alpha(Z \alpha)^{2} \ln (Z \alpha) \\
\alpha(Z \alpha)^{2} \\
\alpha(Z \alpha)^{2} \\
- \\
- \\
(Z \alpha)^{2} m / M \\
\text { or } \alpha(Z \alpha) m / m_{p} \\
\left(Z \alpha m c R_{N} / \hbar\right)^{2}\end{array}$ & $\begin{array}{c}\alpha^{3} \ln \alpha \\
\alpha^{3} \\
\alpha^{3}\end{array}$ & $\begin{array}{c}1 \\
m / M \\
(Z \alpha)^{2} \\
(Z \alpha)^{2} m / M \\
\alpha(Z \alpha)^{2} \ln (Z \alpha) \\
\alpha(Z \alpha)^{2} \\
\alpha \ln \left(Z \alpha m / m_{e}\right) \\
- \\
- \\
(Z \alpha)^{2} m / M \\
\text { or } \alpha(Z \alpha) m / m_{p} \\
\left(Z \alpha m c R_{N} / \hbar\right)^{2}\end{array}$ \\
\hline
\end{tabular}

\section{Table 1}

Various contributions to the energy levels. The results are in units of $(Z \alpha)^{2} m c^{2}$, where $m$ is the mass of the orbiting particle. Here: $M$ is the nuclear mass and $m_{p}$ is the proton mass which enters equations if one measure the nuclear magnetic moment in units of the nuclear magneton. A contribution of the nuclear magnetic moment, i.e., the hyperfine structure, appears if the nuclear spin is not zero. $R_{N}$ stands for the nuclear (charge) radius.

We summarize the most important corrections to the levels determined by Eq. (3) in Table 1. The main phenomena essentially contributing to the structure of the energy levels in hydrogen-like atoms are listed below.

\section{- Non-relativistic consideration}

- The dominant contribution is determined by the Schrödinger equation for a particle with mass $m$ bound by a central Coulomb field. The result has order $(Z \alpha)^{2} m c^{2}$ and we discuss all other corrections in units of $(Z \alpha)^{2} m c^{2}$.

- The leading non-relativistic correction is already incorporated into Eq. (3) by the introduction of the reduced mass (4). The correction has a frac- 
tional order of $m / M$, and the result, presented in Eq. (3), is already corrected. This correction is responsible, for example, for the isotopic shift of hydrogen and deuterium gross structure. In electronic atoms, the correction has order $m_{e} / M \simeq 5 \cdot 10^{-4} / A$, where $A$ is the atomic number (i.e., the atomic mass in the universal atomic mass units). The isotopic shift between atoms with atomic numbers $A$ and $A^{\prime}=A+\Delta A$ involves for heavy elements an additional small factor of $\Delta A / A$. In muonic atoms the correction is of order of $0.1 / A$ and in positronium the effect reduces the result in the leading order by a factor of two.

The result (3) depends on a principal quantum number $n$ only. The structure of levels due to the non-relativistic approximation does not have a single common notation. It used to be named as the gross structure, the main structure, the Rydberg structure, the basic structure etc.

\section{- Relativistic effects}

- The leading relativistic corrections are determined by a solution of the Dirac equation

$$
E_{D}=m c^{2} \times F_{D}\left(n l_{j}\right)
$$

where

$$
F_{D}\left(n l_{j}\right)=\left(1+\frac{(Z \alpha)^{2}}{\left(n-j-1 / 2+\sqrt{(j+1 / 2)^{2}-(Z \alpha)^{2}}\right)^{2}}\right)^{-1 / 2}
$$

and thus

$$
E_{D}=m c^{2} \times\left[1-\frac{(Z \alpha)^{2}}{2 n^{2}}-\frac{(Z \alpha)^{4}}{2 n^{3}}\left(\frac{1}{j+1 / 2}-\frac{3}{4 n}\right)+\ldots\right] .
$$

The first term above is for the rest energy, the next is the leading nonrelativistic term and the third term (of order of $(Z \alpha)^{4} m c^{2}$ in absolute units, or $(Z \alpha)^{2}$ in units of $(Z \alpha)^{2} m c^{2}$ which we apply in this section) is the relativistic correction. This relativistic correction splits levels with the same $n$, but different values of the angular momentum $j$. However, the result does not depend on the orbital momentum $l$. In particular, these relativistic effects are responsible for the fine structure in the hydrogen atom. They split the $2 p_{3 / 2}$ and $2 p_{1 / 2}$ states, but the energy of the $2 s_{1 / 2}$ and $2 p_{1 / 2}$ is still the same.

- The Dirac equation itself is an equation ignoring the nuclear motion. Both the nonrelativistic reduced-mass effects and the leading relativistic recoil correction, which contributed in order $(Z \alpha)^{2} m / M$, can be expressed in terms of the Dirac energy (see, e.g., [15])

$$
\Delta E_{\mathrm{rec}}=m c^{2} \times\left[\frac{m_{R}-m}{m}\left(F_{D}-1\right)-\frac{m_{R}^{3}}{2 M m^{2}}\left(F_{D}-1\right)^{2}\right] \text {. }
$$


We note that a simple substitution $m \rightarrow m_{R}$ reproduces the correct results for the leading terms for both gross and fine structure since only the first term in (8) contributes there. However, the substitution leads to an incorrect result for the leading relativistic correction for, e.g., the $1 s$ state, where the second term in (8) is also important.

- Contributions involving the nuclear structure effects appear already in the non-relativistic approximation.

- One of the main effects is the hyperfine structure, a result of interaction of the nuclear spin $I$ with the angular momentum of the orbiting particle $j$. The energy of the levels depends on the quantum number $F$ (where $\mathbf{F}=\mathbf{j}+\mathbf{I})$. The result is of order of $(Z \alpha)^{2} \mathrm{~m} / M$ if we use the notation of the particle physics, where considers a nucleus as a particle of a charge $Z e$ and mass $M$ and measures its magnetic moment in units of its own magneton $Z e \hbar / 2 M$. Here $e$ is the proton charge. This is the case of positronium and muonium.

In the case of a compound nucleus (deuterium, tritium, helium-3 etc.) another convention is in use. The magnetic moment is measured in units of the nuclear magneton $e \hbar / 2 m_{p}$, where $m_{p}$ is the proton mass. In such a case one usually speaks about a hyperfine structure of order of $\alpha(Z \alpha) m / m_{p}$.

Indeed, these two notations are related to the same order of magnitude since $Z / M \simeq Z /\left(A m_{p}\right) \sim 1 / m_{p}$ with $Z \leq A<3 Z$. In the case of the hydrogen atom these two notations are completely identical.

The hyperfine structure has the same parametrical order for states with any $l$ and $j$, however, the result for the $s$ states is usually considerably larger numerically.

A value of the nuclear magnetic moment is a consequence of its nuclear structure and in conventional atoms (hydrogen, deuterium etc.) cannot be calculated $a b$ initio in contrast to muonium and positronium.

- In the leading order, effects of a distribution of the nuclear charge (socalled finite-nuclear-size effects) shift energy levels of the $s$ states only and the correction has order $\left(Z \alpha m c R_{N} / \hbar\right)^{2}$, where $R_{N}$ is a characteristic nuclear radius.

- Quantum Electrodynamics offers a variety of effects and their hierarchy depends on a kind of atom.

- The leading QED effect in the hydrogen atom (and other conventional atoms) is the self energy contribution (see Fig. 2) which leads to a correction of order $\alpha(Z \alpha)^{2} \ln (1 /(Z \alpha))$. It splits the levels with the same $j$, but different values of $l$. An example is a splitting of $2 s_{1 / 2}-2 p_{1 / 2}$ (the Lamb shift).

- The vertex diagram in presence of the magnetic field is chiefly responsible for a correction to the magnetic moment (i.e., for the anomalous magnetic moment of an electron) and other effects are of higher order. As we already mentioned, the anomalous magnetic moment of an electron was first detected as an anomaly in the hyperfine structure of atomic hydrogen.

- An electron vacuum polarization (see Fig. 3) in the hydrogen atom is 


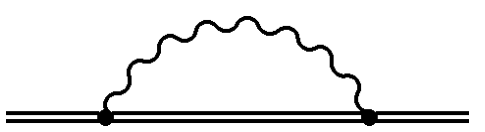

Fig. 2. The self-energy diagram which is responsible for the dominant contribution to the splitting of $2 s_{1 / 2}-2 p_{1 / 2}$ (the Lamb shift) in the hydrogen atom. The doubled electron line is related to the Green function of an electron in the external Coulomb field.

responsible for a small fraction of the Lamb shift. In a general case, the correction, also called the Uehling correction, has order $\alpha F\left(Z \alpha m / m_{e}\right)$, where

$$
F(x) \sim \begin{cases}x^{2}, & x \ll 1 \\ 1, & x \sim 1 \\ \ln x, & x \gg 1\end{cases}
$$

In the conventional atoms, where $m_{e}=m$ and $x=Z \alpha$, the Uehling contribution does not change the hierarchy of the intervals. In muonic atoms, where $x \simeq 1.5 Z$, the Uehling correction can produce $2 s-2 p$ splittings bigger than $2 p_{3 / 2}-2 p_{1 / 2}$. The hierarchy depends on $Z$.

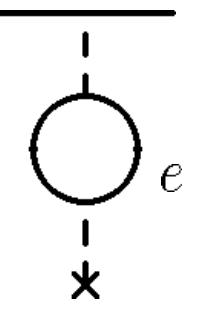

Fig. 3. The vacuum polarization diagram (the Uehling contribution). In the light muonic atoms it leads to the dominant contribution to the splitting of $2 s-2 p$ (the Lamb shift) at low and medium $Z$.

- In the case of positronium the two effects above are not so important as the virtual annihilation of the bound electron and positron into a single photon (see Fig. 4). The one-photon annihilation splits two hyperfine levels related to the $S$ states: the ${ }^{3} S_{1}$ state (triplet) is shifted while the ${ }^{1} S_{0}$ state (singlet) is not affected.

- The QED effects are also responsible for decay of atomic states (see Fig. 5). The radiative decay line width is basically $\alpha(Z \alpha)^{2}$. This result is related to the one-photon electric-dipole transition (so called $E 1$ transition) which is a dominant decay mode for all levels in light hydrogen-like atoms except the $2 s$ and $1 s$ states. The upper hyperfine component of the $1 s$ states can decay via a magnetic-dipole $(M 1)$ transition with an extremely small line width while the metastable $2 s$ state decays via a two-photon electric-dipole $(2 E 1)$ transition with the line width of order of $\alpha^{2}(Z \alpha)^{4}$. 


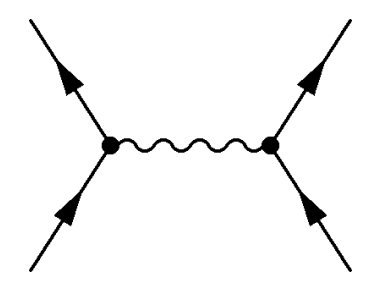

Fig. 4. The one-photon annihilation diagram. The real annihilation into a single photon is not possible for kinematic reasons, while the virtual one is possible and it shifts the energy levels of $s$ states with total spin 1 .

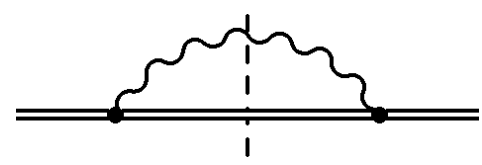

Fig. 5. The imaginary part of the self energy diagram is the energy width of the level and thus it is equal to the probability of its radiative decay via emission of a single photon.

- In the case of positronium the line width of the $S$ states $^{1}(n=1,2)$ depends on their total angular momentum (spin). The triplet states $\left({ }^{3} S_{1}\right)$ are long living decaying via the three-photon annihilation, while the twophoton annihilation is a dominant mode for the short-living singlet states $\left({ }^{1} S_{0}\right)$. The widths are $\alpha^{4}$ and $\alpha^{3}$ respectively. Higher $S$ states additionally to annihilation modes can also decay radiatively into $P$ states (one photon $E 1$ transition) with a related width of order of $\alpha^{3}$. In the case of non- $S$ states $(P, D$ etc.) the radiative decay dominates.

In any case the contribution in Eq. (3) dominates for an interval between levels with $\Delta n \neq 0$. However, the structure of levels with the same value of $n$ depends on details of the atom. In Fig. 6 we present three different basic spectra of the structure at $n=2$.

- The first one is realized in 'normal' (electronic) hydrogen-like atoms (hydrogen, deuterium, helium ions etc.). The muonium spectrum is of the same kind. The largest splitting, of order $(Z \alpha)^{4} m_{e} c^{2}$, is the fine structure (i.e., a splitting between levels with a different value of the electron angular momentum $j$ ), the Lamb shift arising from the electron self-energy effects is of

1 We use the low case letters $(s, p$ etc.) for single-electron atoms, while the capital letters are used for atoms with a few electrons. Other indexes for single-electron atoms are related to the electron properties, while a total angular momentum $F$ is indicated in the parentheses: e.g., $1 s_{1 / 2}(F=1)$. In the case of positronium, since the masses of electron and nucleus are the same, we use capital letters indicating that we deal with the total atomic momentum related to two particles and the subscripts and superscripts are related to properties of an atom as a whole. 

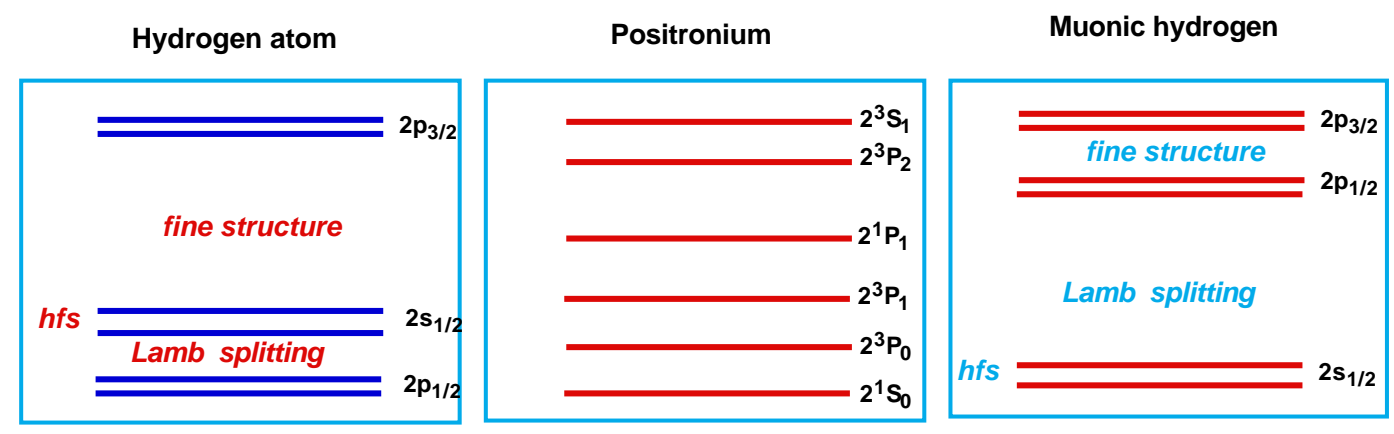

Fig. 6. Scheme of the lowest excited levels $(n=2)$ in different simple atoms (not to scale).

order $\alpha(Z \alpha)^{4} m_{e} c^{2} \ln (1 /(Z \alpha))$ and it splits the levels with the same $j$ and different values of the electron orbital momentum $l$. Some nuclei are spinless (like, e.g., $\left.{ }^{4} \mathrm{He}\right)$, while others have a non-zero spin $(I=1 / 2$ in hydrogen, muonium, helium-3 and $I=1$ in deuterium etc.). In the latter case, the interaction with the nuclear spin splits levels with the same electronic quantum number. The splitting is of order $(Z \alpha)^{4} m_{e}^{2} c^{2} / M$ or $\alpha(Z \alpha)^{3} m_{e}^{2} c^{2} / m_{p}$, where $M$ is the nuclear mass, and the structure depends on the value of the nuclear spin. The scheme in Fig. 6 is for a nuclear spin of 1/2. A difference for higher $I$ is that the states with $j \geq 1$ ( such as $2 p_{3 / 2}$ with $j=3 / 2$ ) are split in three or more subcomponents. In a general case, the number of hyperfine components is the smaller number among $2 j+1$ and $2 I+1$.

- The structure of levels in positronium and muonic atoms is different because other QED effects enter consideration. For positronium, an important feature is a real (into two and three photons) and virtual (into one photon) annihilation. The former is responsible for the decay of the $s$-states, while the latter shifts triplet $s$-levels $\left(1^{3} S_{1}\right.$ and $2^{3} S_{1}$ in particular). The shift is of the order of $\alpha^{4} m_{e} c^{2}$. Contributions of the same order arise from relativistic effects for the both, the electron and the nucleus (namely, positron), and from hyperfine interactions. As a result, the structure of the positronium levels at $n=2$ has no apparent hierarchy (Fig. 6).

- Another situation is for the spectrum of the muonic atoms. A difference to the hydrogen case comes from a contribution due to the vacuum polarization effect (the Uehling potential). Effects of electronic vacuum polarization shift all levels to the order of $\alpha(Z \alpha)^{2} m_{\mu} c^{2}$. This shift is a nonrelativistic one and it splits $2 s$ and $2 p$ levels, but in does not depend on $j$ and cannot split $2 p_{3 / 2}$ and $2 p_{1 / 2}$. The fine and hyperfine structures are of the same form as for the normal atoms (i.e., $(Z \alpha)^{4} m_{\mu} c^{2}$ and $(Z \alpha)^{4} m_{\mu}^{2} c^{2} / M$, respectively), but the latter is much more important than in a conventional atom since $m_{\mu} / m_{p} \sim 1 / 9$. At the case of low $Z$ the Lamb shift induced by the Uehling potential is the dominant correction to the energy levels.

Table 1 shows $Z$ dependence of various crucial contributions to energy of bound states in two-body atoms and to their decay width. Since the radiative 
linewidth increases with $Z$, it is much easier to work with light atoms which provide a longer lifetime of excited states. We note, however, that importance of QED effects also increases with $Z$ in such a way that a ratio of a correction to width, which is of interest, rather favors heavy ions with high $Z$. Meanwhile there is a number of other reasons which make low- $Z$ atoms more attractive.

- Studies of neutral atoms and ions involve quite different methods and as a result, some quantities are better determined for neutral two-body atoms (like, e.g., the hyperfine interval in the ground state) while others lead to a higher accuracy for ions (like, e.g., the hyperfine interval for the excited $2 s$ state in helium-ion ${ }^{3} \mathrm{He}^{+}$). Indeed, all neutral two-body atoms have a low value of the nuclear charge, namely, $Z=1$.

- Precision QED tests are realized in atomic systems which can be calculated $a b$ initio. Such a theory can be in principle developed for any QED effects, but not for the nuclear structure. Importance of the nuclear structure effects increases with $Z$. In the leading non-relativistic approximation (see Table 1) the related nuclear-spin-independent contribution to the energy is proportional to $Z^{4}$ and a squared value of the nuclear charge radius which also increases with $Z$. In the case of the nuclear-spin-dependent contributions (hyperfine effects) the related correction is proportional in the non-relativistic approximation to $Z^{5}$ and to a characteristic value of the nuclear size. In the case of high $Z$ the non-relativistic approximation still leads to a correct order of magnitude, however, the dependence on the nuclear charge and nuclear radii becomes more complicated.

As a consequence, studying hyperfine interactions one has either to study the nucleon-free atoms (muonium, positronium) or try to cancel the nuclear effects between a few measured quantities. That is possible because low$Z$ atoms allow to present the nuclear effects in terms of a few effective parameters (such as the nuclear charge radius, nuclear magnetic radius etc.). On contrary, for highly charged ions we rather need to apply certain models of distribution of their charge and magnetic moment and thus the QED tests with high- $Z$ ions become model-dependent, in comparison with modelindependent calculations for low- $Z$.

- The Rydberg constant is related to an ultraviolet part of spectrum. Thus, at low $Z(Z=1,2)$ there is a good chance to study some transitions in gross structure with lasers while for higher $Z$ laser spectroscopy can be used only for the fine structure and the Lamb shift as well as for muonic atoms. Strong dependence of the transition frequency on $Z$ allows only a few opportunities for a spectrum of two-body atoms with $Z \neq 1$ and/or $m \neq m_{e}$.

- In contrast, a number of transitions in neutral atoms $Z=1$ are suited for laser spectroscopy. That is not a surprise. The characteristic atomic and molecular energies we meet in our life are of the same order as in hydrogen since they are mostly related to certain single-electron excitations in a Coulomb field of an effective charge of $Z_{\text {eff }} \sim 1$. Since we are forced to meet them in our common-day, industrial and scientific life the most 
advanced radiation sources and useful tools were developed for these regions (optical with some inclusion of infrared and ultraviolet domains). Their development has a history of a few centuries of making lenses, mirrors, prisms etc.

- As we noted, the ratio of the crucial QED corrections of interest (e.g., $\alpha^{2}(Z \alpha)^{6} m c^{2}$ contributions to the Lamb shift) and the lifetime (which scales for the $E 1$ transitions such as $2 p \rightarrow 1 s$ as $\left.\alpha^{5}(Z \alpha)^{4} m c^{2}\right)$ clearly favors higher values of $Z$. However, that is not a whole story. First, there is a metastable $2 s$ state in hydrogen-like atoms which lifetime scales as $\alpha^{2}(Z \alpha)^{6} m c^{2}$ and use of this level is crucial for a number of experiments. Because of the longer lifetime and a proper value of the $1 s-2 s$ interval it is much easier to deal with this level at low $Z$. In the hydrogen atom the $2 p$ lifetime is about $10 \%$ of the Lamb splitting $2 s_{1 / 2}-2 p_{1 / 2}$ while a much smaller value of the natural $2 s$ lifetime cannot be even seen from the line shape of the twophoton $1 s-2 s$ excitation in precision spectroscopy experiments because of various perturbations which significantly shorten the $2 s$ lifetime (which is still much longer than that of the $2 p$ state). The narrow $1 s-2 s$ transition was successfully used in a number of hydrogen experiments while there is no access to this narrow transition for high $Z$. Secondly, the lifetime of the E1 width sharply depends not only on a value of the nuclear charge $Z$ but also on the principal quantum number $n$. With such an $n$-dependence which can be roughly approximated as $1 / n^{3}$ one can realize that the $2 p$ transition is one of the broadest. The high- $Z$ experiments used to deal with $2 s-2 p$ and $1 s-2 p$ intervals and thus the involvement of the $2 p$ state is unavoidable. On contrary, the recent success in hydrogen spectroscopy is related to high $n$ levels studying $2 s-n d$ and $2 s-n s$ at $n=8-12$. Use of the metastable $2 s$ level and highly excited levels in hydrogen compensates all disadvantages of the correction-to-width ratio for low $Z$ and makes the low- $Z$ case most favorable for the bound state QED tests.

Since experimental and theoretical methods favor the light atoms, we focus our consideration of particular QED tests on hydrogen and other low $Z$ atoms and touch other atoms only in case if they provide any results competitive with $Z=1$ atomic systems.

\section{Optical measurements in hydrogen atom and determination of the Rydberg constant}

About fifty years ago it was discovered that in contrast to the spectrum predicted by the Dirac equation, there are some effects in hydrogen atom which split the $2 s_{1 / 2}$ and $2 p_{1 / 2}$ levels. Their splitting known now as the Lamb shift (see Fig. 7) was successfully explained by quantum electrodynamics. The QED effects lead to a tiny shift of energy levels and for thirty years this shift was 
studied by means of microwave spectroscopy (see, e.g., [31,32]) measuring either directly the $2 s_{1 / 2}-2 p_{1 / 2}$ splitting or the larger $2 p_{3 / 2}-2 s_{1 / 2}$ splitting (fine structure) where the QED effects are responsible for approximately $10 \%$ of the interval.

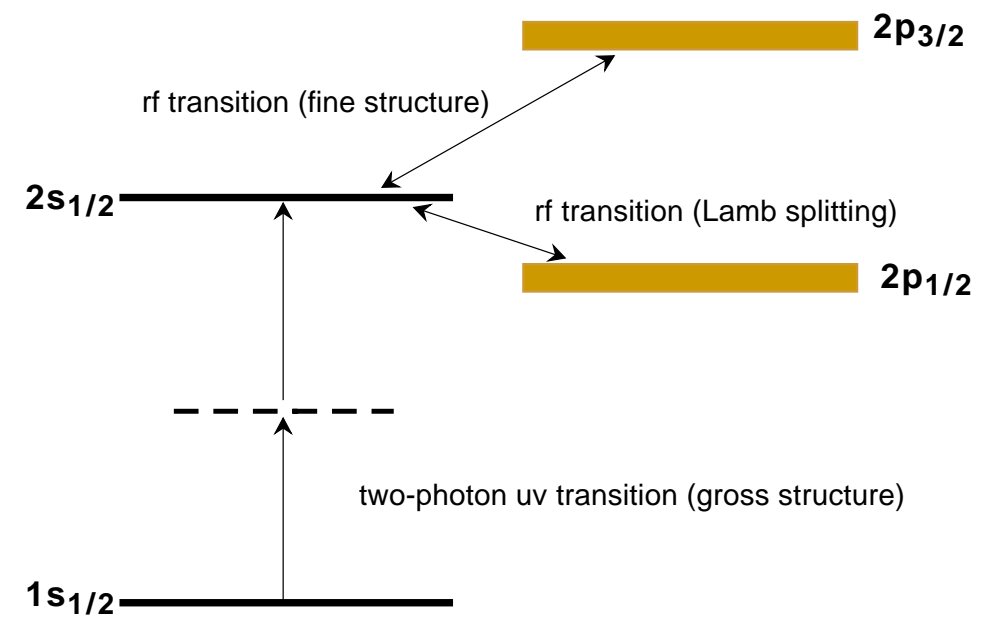

Fig. 7. An example of different transitions in the hydrogen atom (not to scale). Transitions within the fine structure and Lamb splitting are in the microwave range, while the $1 s-2 s$ two-photon transition lies in the ultraviolet domain. The Lamb splitting is the difference of the Lamb shifts of $2 s_{1 / 2}$ and $2 p_{1 / 2}$ levels. The hyperfine structure is neglected. The $2 p$ linewidth is approximately $10 \%$ of the Lamb shift, which itself is approximately a tenth part of the fine structure splitting.

A recent success of two-photon Doppler-free spectroscopy [33] opens another way to study QED effects directed to high-resolution spectroscopy of grossstructure transitions. The energy of such a transition between levels with different values of the principal quantum number $n$ is mainly determined by the Coulomb-Schrödinger formula in Eq. (3). However, an unprecedently high accuracy achieved by to date allows to study various tiny perturbations to Eq. (3), caused by effects of relativistic physics, quantum electrodynamics and nuclear structure. All studied transitions are related to the ultraviolet part of the spectrum.

For any interpretation of hydrogenic lines in terms of QED effects one has to determine a value of the Rydberg constant

$$
R_{\infty}=\frac{\alpha^{2} m c}{2 h}
$$

One more problem for the interpretation of the optical measurements is the involvement of few levels, significantly affected by the QED effects. In contrast to radiofrequency measurements, where the $2 s-2 p$ splitting was under study, the optical measurements have been performed with several transitions involving a number of states $(1 s, 2 s, 3 s$ etc.). It has to be noted that the Lamb 
shift for levels with $l \neq 0$ has a relatively simple theory, while theoretical calculations for the $s$ states lead to several serious complications. The problem has been solved by introducing an auxiliary specific difference [34,35]

$$
\Delta(n)=E_{L}(1 s)-n^{3} E_{L}(n s)
$$

for which theory is significantly simpler and more clear than for each of the $s$ states separately.

Combining theoretical results for the difference $[35,36]$ with a measured frequency of two or more transitions one can extract a value of the Rydberg constant and the Lamb shift in the hydrogen atom.

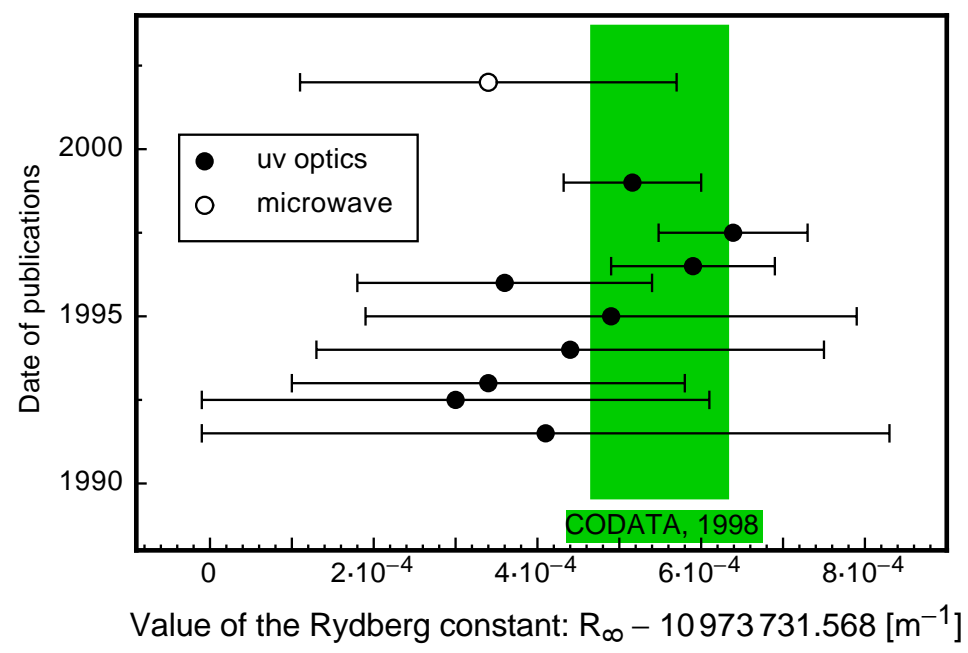

Fig. 8. Progress in determination of the Rydberg constant by means of two-photon Doppler-free spectroscopy of hydrogen and deuterium. The label CODATA stands for the recommended value of the Rydberg constant $R_{\infty}$ (1998) [21] from Eq. (12). The most recent original value is a preliminary result from MIT obtained by microwave means [37].

The recent progress in determination of the Rydberg constant is presented in Fig. 8 (see Refs. [33,21] for references). The progress is also clearly seen from the recommended CODATA values of 1986 [20] and 1998 [21]:

$$
R_{\infty}(1986)=10973731.534(13) \mathrm{m}^{-1}
$$

and

$$
R_{\infty}(1998)=10973731.568549(83) \mathrm{m}^{-1} .
$$

The former value was derived from one-photon transitions (Balmer series) and was 'slightly' improved later (by a factor of 4.5), but all further progress that 
led to the 1998's value (improvement by more than 30 times) was a result of the study of two-photon transitions in hydrogen and deuterium. Figure 8 shows a comparison of several recently published values for the Rydberg constant.

As mentioned before, the other result, which can be extracted from the optical spectroscopy of the hydrogen atom, is the $1 s(2 s)$ Lamb shift. However, studies of the Rydberg constant and the Lamb shift, which are related quantities, are still not the same. While the optical measurements deliver us the most accurate data on both values (and in fact the data are strongly correlated), the microwave experiments still supply us with various less precise data on both quantities and the microwave data on the Lamb shift and the Rydberg constant are completely independent from each other.

The only example of a microwave determination of the Rydberg constant is an MIT experiment [37], which dealt with highly excited hydrogen levels. The transitions under study were between $n=30$ and $n=27$ for high $l$ and because of such high values of $n$ and $l$ the levels were not very sensitive to the QED effects and thus the derived value of the Rydberg constant is 'Lamb-shift-free'. The preliminary result [37]

$$
R_{\infty}=10973731.56834(23) \mathrm{m}^{-1}
$$

agrees with other values (see Fig. 8). All other important related microwave results in the field have been achieved for either the Lamb shift or the fine structure.

\section{The Lamb shift in the hydrogen atom}

After the progress of the last decade, the optical determination of the Lamb shift [33] superseded traditional microwave measurements [31,32], but still there is a number of compatible microwave results among the published data. The experimental results are summarized in Fig. 9.

To discuss the state of the art in study of the Lamb shift in detail let us start with the experimental data. A number of different intervals in the hydrogen atom have been precisely studied for about a century. Bound state QED and in part even QED itself were established essentially in order to explain the Lamb shift and the fine and hyperfine structure in the hydrogen atom as well as in the deuterium atom and the helium ion ${ }^{2}$. The corresponding transitions lie in the

2 The Lamb shift is sometimes named after Lamb and Retherford, referring to their famous papers on the Lamb shift in the hydrogen atom [40]. However, that is not quite correct, since at approximately the same time a result on the Lamb shift in 


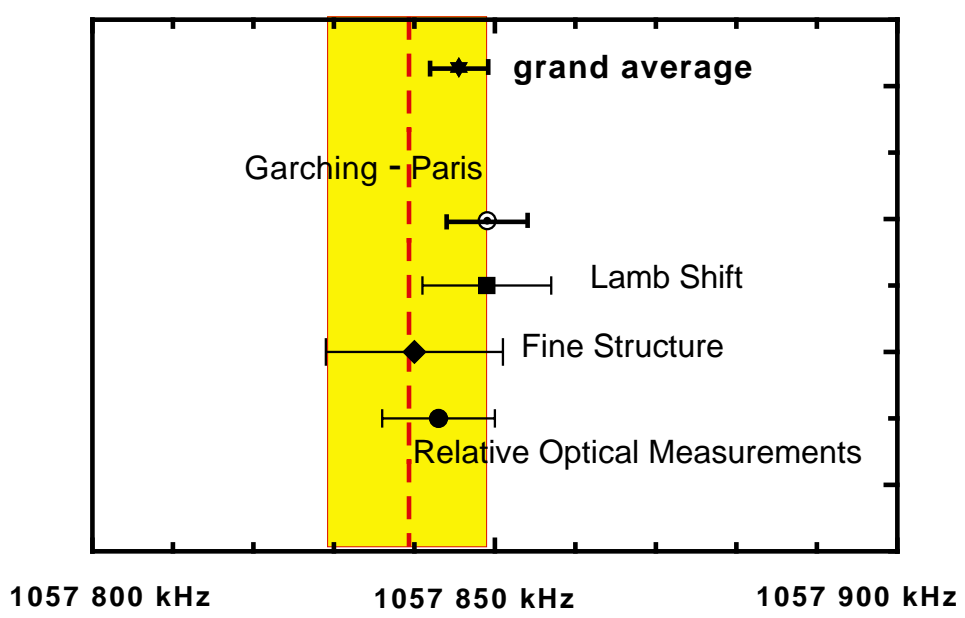

Fig. 9. Measurement of the Lamb shift in the hydrogen atom. The most accurate experimental result comes from a comparison of the $1 s-2 s$ interval measured at MPQ (Garching) [38] and the $2 s-n s / d$ intervals at LKB (Paris) [39], where $n=8,10,12$ (see also [33] for detail). Three more results are shown for the average values extracted from direct Lamb shift measurements, measurements of the fine structure and a comparison of two optical transitions within a single experiment (i.e., a relative optical measurement). The filled part is for theory. Theory and evaluation of the experimental data are presented according to Ref. [36].

microwave part of the spectrum. In the last decades, progress in radiofrequency experiments with hydrogen and other light atoms was rather slow. The results of over than thirty years related to the Lamb shift in hydrogen are presented in Fig. 10 (measured directly) and Fig. 11 (the Lamb shift deduced from the measured fine structure interval $2 p_{3 / 2}-2 s_{1 / 2}$ ). The most recent results are presented separately, while the older experiments are averaged. The highest accuracy was claimed in [45] and we correct here their value according to $[34,46]$. Nevertheless, the result is not included in the Lamb shift average value in Fig. 9 (the $L S$ value) because of unclear status of its uncertainty, which is discussed in part below in this section.

To reach a value of the Lamb shift from the fine structure $\left(2 p_{3 / 2}-2 s_{1 / 2}\right)$ measurement we need to use a value of the $2 p_{3 / 2}-2 p_{1 / 2}$ splitting which was found theoretically

$$
\begin{aligned}
\Delta E_{L}\left(2 p_{j}\right) & =\Delta E_{L}(2 p)+\frac{(Z \alpha)^{4} m c^{2}}{8} \times\left\{a_{e} \cdot A_{40}^{a}\left(\frac{m_{R}}{m}\right)^{2}+C_{R}\left(\frac{m}{M}\right)^{2}\right. \\
& \left.+\frac{\alpha(Z \alpha)^{2}}{\pi}\left(A_{61} \ln \frac{1}{(Z \alpha)^{2}}+A_{60}^{V P}+G_{S E}(Z \alpha)\right)\right\} \\
& + \text { higher order terms }
\end{aligned}
$$

the helium ion was presented in a paper by Skinner and Lamb [41]. 


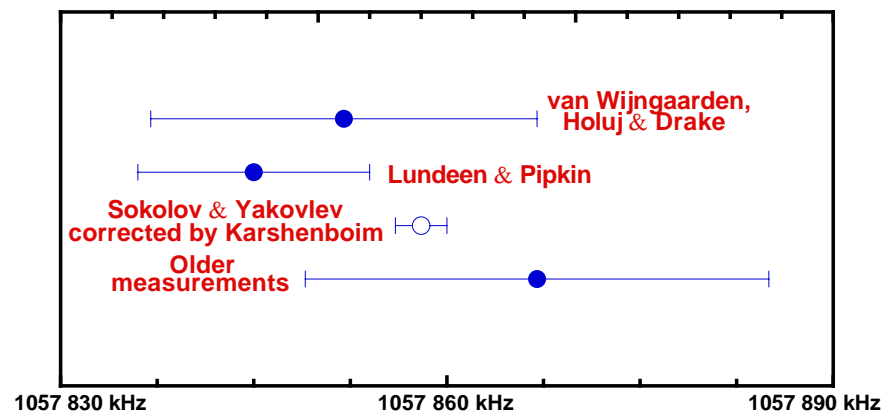

Fig. 10. Direct measurement of the Lamb shift in the hydrogen atom. The most recent result was obtained in [42,43], while the older results [44] are averaged. The result of Sokolov and Yakovlev [45] has been corrected according to [46].

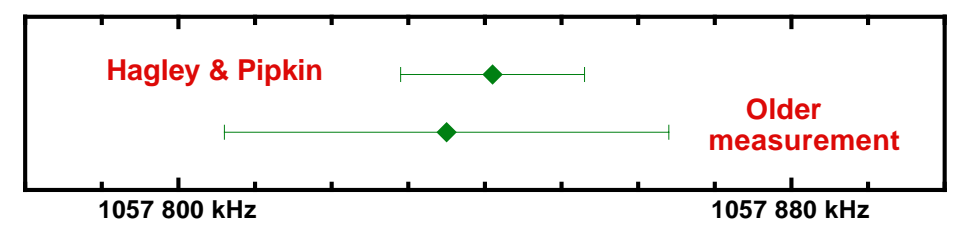

Fig. 11. An indirect determination of the Lamb shift $\left(2 s_{1 / 2}-2 p_{1 / 2}\right)$ in atomic hydrogen via a study of the fine structure $2 p_{3 / 2}-2 s_{1 / 2}$. The most recent result was obtained in [47], the older results [48] are averaged.

where we introduce a $j$-independent part of the Lamb shift of the $2 p$ state

$$
\begin{aligned}
\Delta E_{L}(2 p) & =\frac{\alpha(Z \alpha)^{4}}{8 \pi} m c^{2}\left(\frac{m_{R}}{m}\right)^{3}\left\{-\frac{4}{3} \ln k_{0}(2 p) \times\left(1+\frac{Z m}{M}\right)^{2}\right. \\
& \left.+\frac{\alpha(Z \alpha)^{2}}{\pi} \ln ^{2} \frac{1}{(Z \alpha)^{2}} B_{62}+\frac{Z m}{M}\left(C_{\text {Rec }}+\frac{\pi}{3}(Z \alpha)\right)\right\} \\
& + \text { higher order terms } .
\end{aligned}
$$

The state-dependent coefficients $A_{40}^{a}, A_{61}, A_{60}^{V P}, C_{\mathrm{Rec}}, C_{R}$ and $B_{62}$ and the function $G_{S E}(Z \alpha)$ are well known (see in Table 2); $a_{e}$ stands for the anomalous magnetic moment of the electron and $\ln k_{0}(2 p)=-0.030016709 \ldots$ is the Bethe logarithm.

The direct measurements of the Lamb splitting (i.e., the interval between $2 s_{1 / 2}$ and $2 p_{1 / 2}$ ) need in principle no auxiliary QED calculations. An exception is the result of experiment [45] where the originally measured quantity was a product of the $2 s_{1 / 2}-2 p_{1 / 2}$ splitting and the lifetime of the $2 p_{1 / 2}$ state. The measurement was claimed to be the most accurate determination of the Lamb shift (the assigned uncertainty was about $2 \mathrm{ppm}$ ). The result [45] has been corrected due to a recalculation of the lifetime $[34,46]$, where the leading radiative correction was taken into account within a logarithmic approximation 


\begin{tabular}{lccc}
\hline Coefficients & $\Delta E_{L}\left(2 p_{1 / 2}\right)$ & $\Delta E_{L}\left(2 p_{3 / 2}\right)$ & $\Delta(2)$ \\
\hline$A_{40}^{a}$ & $-\frac{1}{3}$ & $\frac{1}{6}$ & 0 \\
$A_{61}$ & $\frac{2}{9}+\frac{7}{20}$ & $\frac{1}{10}+\frac{2}{9}$ & $4 \ln 2-\frac{197}{60}$ \\
$A_{60}^{\mathrm{VP}}$ & $-\frac{9}{140}$ & $-\frac{1}{70}$ & $\frac{4}{15} \ln 2+\frac{1}{140}$ \\
$G_{\mathrm{SE}}$ & $-0.9735(2)$ & $-0.4865(2)$ & $0.89491(10)$ \\
$B_{62}$ & $\frac{1}{9}$ & $\frac{1}{9}$ & $\frac{16}{9} \ln 2-\frac{7}{3}$ \\
$C_{R}$ & $\frac{1}{6}-C_{\text {mix }} \times \delta_{F 1}$ & $-\frac{1}{12}+C_{\text {mix }} \times \delta_{F 1}$ & 0 \\
$C_{\operatorname{Rec}}$ & $-\frac{7}{18}$ & $-\frac{7}{18}$ & $\frac{14}{3}\left(\ln 2-\frac{3}{4}\right)$ \\
\hline
\end{tabular}

Table 2

Coefficients for higher-order contributions to the hydrogen Lamb shift $\Delta E_{L}(2 p)$ and specific difference $\Delta(2)$ (see Eq. (11)). Most of the coefficients are universal and are the same for, e.g., deuterium, with one exception: coefficient $C_{R}$ depends on the nuclear spin, it is presented for hydrogen $(I=1 / 2)$; coefficient $C_{\text {mix }}=\left(g_{p}\right)^{2} / 162$ is due to mixture of the $2 p_{1 / 2}(F=1)$ and $2 p_{3 / 2}(F=1)$ states by the hyperfine interaction and was considered in [49] (see, also [50]) and $F$ is the complete angular momentum and its value for the $2 p_{1 / 2}$ state in hydrogen can be either 0 or 1 , while for the $2 p_{3 / 2}$ state it can take value of 1 or 2 .

$$
\begin{aligned}
\tau^{-1}\left(2 p_{1 / 2}\right)= & \frac{2^{9}}{3^{8}} Z^{4} \alpha^{3}\left(2 \pi \cdot c R_{\infty}\right) \frac{m_{R}}{m} \times\left(1+(Z-1) \frac{m}{M}\right)^{2} \times \\
& \left\{1+(Z \alpha)^{2} \ln \left(\frac{9}{8}\right)\right. \\
+ & \frac{\alpha(Z \alpha)^{2}}{\pi}\left[(-2.622 \ldots) \ln \frac{1}{(Z \alpha)^{2}}+C_{0}\right] \\
+ & \text { higher order terms }\} .
\end{aligned}
$$

We remind here that $R_{\infty}$ is related to the wave number $(\nu / c)$, while $c R_{\infty}$ corresponds to frequency $\nu$. Factor of $2 \pi$ appears because $h \nu=\hbar \omega$ and the lifetime $\tau$ is related to the line width of the angular frequency $\omega=2 \pi \nu$.

The logarithmic part of $\alpha(Z \alpha)^{2}$ term was calculated in $[34,46]$ (see also $[51,52]$ ). Recently a non-logarithmic term was also calculated $C_{0}=6.576 \ldots$ [53]. Since our result [52] already included an estimation of the constant $C_{0}=6.2(5)$, that leads to a marginal shift in the value of $C_{0}$, but makes the result previously based on a naive estimation [52] more reliable. We also have to mention that an exact calculation of $C_{0}$ alone is not sufficient for any application because the line width is not well defined. As it is well known, the line shape contributions to the width enter in fractional order $\hbar /[\tau \cdot \Delta E]$, which in the case of an $E 1$ transition in a hydrogen-like atom is related to an $\alpha(Z \alpha)^{2}$ correction. That is of the same order as under question and thus the non-logarithmic term should depend on the phenomenon under study. If we like to express the result 
of the Sokolov's experiment [45] in terms of $\tau\left(2 p_{1 / 2}\right) \cdot\left[E_{L}\left(2 s_{1 / 2}\right)-E_{L}\left(2 p_{1 / 2}\right)\right]$ and to present the effective lifetime $\tau\left(2 p_{1 / 2}\right)$ in terms of Eq. (15), we see that the coefficient $C_{0}$ is phenomenon-dependent. However, we note that a numerically big contribution to the effective constant $C_{0}$ cannot appear in such a way since $\hbar /[\tau(2 p) \cdot \Delta E(2 p-1 s)] \simeq 0.33 \alpha^{3} / \pi$. To be conservative we double the value of $\hbar /\left[\tau(2 p) \cdot \Delta E_{L}(2 p-1 s)\right]$ and estimate the phenomenon-dependent contribution to $\tau\left(2 p_{1 / 2}\right)$ as $\Delta C_{0} \simeq \pm 0.7$ which is to be added to $C_{0}$ for the interpretation of experiment [45].

We have not taken into account the result of [45] when calculating the average values in the summary picture (Fig. 9) because there are certain doubts in its accuracy noted by Hinds [31] and it is not really clear if the result is as accurate as it was stated originally. The result of [45] is rather in a not good agreement with both the theoretical value and a grand average over the other data. The discrepancy with theory does not exceed two standard deviations and it is too early to consider that as a real contradiction.

We need, however, to note that a common opinion on the future of the microwave measurements of the Lamb shift in hydrogen and on the reliability of the result [45] with a 2-ppm uncertainty has assumed two contradicting statements.

- First, it is generally believed that since any $2 s-2 p$ intervals, (either $2 s_{1 / 2}-$ $2 p_{1 / 2}$ (the Lamb splitting) or $2 p_{3 / 2}-2 s_{1 / 2}$ (the fine structure)) have a width of $0.1 \mathrm{GHz}$ (because of the radiative lifetime of the $2 p$ state), the Lamb splitting (which is about $1 \mathrm{GHz}$ ) cannot be measured better than at the $10 \mathrm{ppm}$ level. Thus, it is expected that the statistical error of a microwave measurement should be larger than $10 \mathrm{ppm}$.

- Secondly, sharing the Hinds' point of view [31], it is believed that the accuracy of the Sokolov's experiment [45] is not as high as claimed only because of a possible systematic error in the determination of the atomic beam velocity, which, in principle, might be somehow fixed in the next generation of experiments. Meanwhile, there has been no doubt expressed on the statistical treatment of the Sokolov's data.

Because of this inconsistency in a opinion of experts, the question on a possibility of a further progress of microwave measurements of the Lamb shift with an uncertainty below $10 \mathrm{ppm}$ remains unclear. A measurement of the Lamb shift in deuterium within the Sokolov's scheme could provide a chance to test partly systematic effects since the result may be compared with the hydrogendeuterium isotopic shift of the $1 s-2 s$ interval [54]. The latter, combined with the theoretical result on specific difference in Eq. (11) [34,35,36], leads to the isotopic shift for the Lamb shift 


$$
\begin{aligned}
\left.\Delta E\left(2 s_{1 / 2}-2 p_{1 / 2}\right)\right|_{D} ^{H}= & -\left.\Delta E_{L}\left(2 p_{1 / 2}\right)\right|_{D} ^{H}-\frac{1}{7}\left\{\Delta E_{\exp }(1 s-2 s)\right. \\
& \left.-\Delta E_{\widetilde{\text { Dirac }}}(1 s-2 s)+\Delta(2)\right\}\left.\right|_{D} ^{H}
\end{aligned}
$$

with an uncertainty below $1 \mathrm{kHz}$. Here a calculation of three terms (namely, the Lamb shift of the $2 p_{1 / 2}$ state, the specific difference of Eq. (11) at $n=2$ and the energy related to an effective Dirac equation

$$
\begin{aligned}
E_{\text {Dirac }}=m c^{2} & +m_{R} c^{2} \times\left[\left(F_{D}-1\right)-\frac{m_{R}^{2}}{2 M m}\left(F_{D}-1\right)^{2}\right. \\
& \left.+\frac{(Z \alpha)^{4}}{2 n^{3}} \frac{m_{R}^{4}}{M^{2} m^{2}}\left(\frac{1}{j+1 / 2}-\frac{1}{l+1 / 2}\right)\left(1-\delta_{l 0}\right)\right]
\end{aligned}
$$

which takes into account recoil effects up to $(Z \alpha)^{4} m_{e}^{3} c^{2} / M^{2}$ (the dimensionless Dirac energy $F_{D}\left(n l_{j}\right)$ is defined in Eq. (6)), can be performed with an uncertainty substantially below $1 \mathrm{kHz}$. The only experimental value in the right-hand part of the equation (the isotopic shift of the $1 s-2 s$ frequency) is also known with an uncertainty below $1 \mathrm{kHz}$ [54].

Essential progress in studies of the hydrogen Lamb shift came some time ago from the optical two-photon Doppler-free experiments (see [33,55] for detail). The Doppler-free measurements offer a determination of some transition frequency in the gross structure with an accuracy high enough to use the results to find the Lamb shift. However, the cancellation of the linear Doppler effect is not an only essential advantage of those experiments. A microwave measurement is to be designed to determine an interval between the $2 s$ state and either the $2 p_{1 / 2}$ or $2 p_{3 / 2}$ state which has a broad line width limiting the accuracy of the measurement. It is not absolutely clear what the ultimate limit of the measurements involving the $2 p$ states is, but indeed an experiment with more narrow levels should have a good chance for a higher accuracy. That is the two-photon excitation of the metastable $2 s$ state to higher excited $n s$ and $n d$ levels that allows to deal with some relatively narrow levels because of the $n^{-3}$ dependence of their line width. However, with the expanding number of the involved energy levels, two special problems as we mentioned before have appeared in the optical experiments: a determination of the Rydberg constant and needs to reduce the variety of the QED contributions for a number of levels to a single quantity, e.g., to the Lamb shift of the $2 s$ (or $1 s$ ) state $E_{L}(2 s)$.

Two methods were applied to manage the problem with the Rydberg contribution. The first of them was for a measurement of two different frequencies within one experiment with the ratio of the frequencies being approximately an integer number. Extracting a beat frequency one can avoid the problem of determining the Rydberg constant at all. Three experiments have been per- 
formed in this way: the Garching experiment dealt with the $1 s-2 s$ transition and the $2 s-4 s$ (and $2 s-4 d$ ) transition [56], at Yale the $1 s-2 s$ frequency was compared with the one-photon $2 s-4 p$ transition [57] and actually that was the only precision optical experiment with an one-photon transition among the recent generation of the experiments on hydrogen spectroscopy. More recent Paris experiment dealt with the $1 s-3 s$ and $2 s-6 s$ (and $6 d$ ) intervals [58]. The values derived from those experiments are collected in Fig. 12.

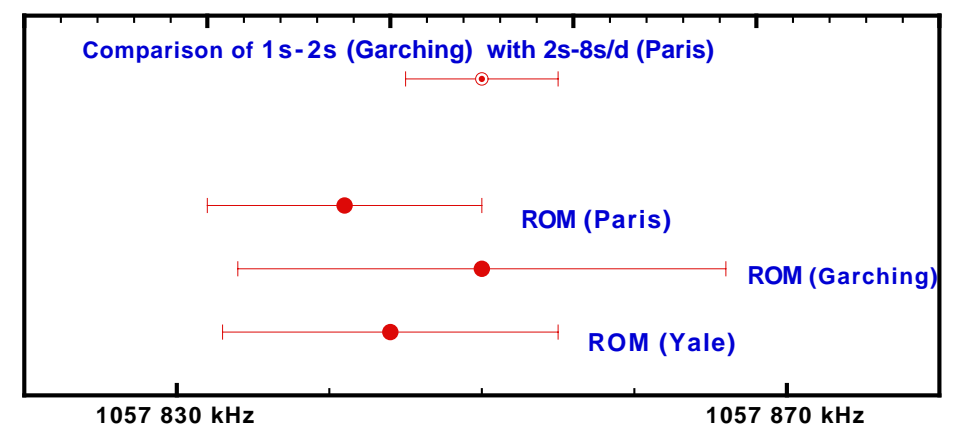

Fig. 12. An optical determination of the Lamb shift in the hydrogen atom. The label $R O M$ stands for relative optical measurements, which dealt with a comparison of two optical frequencies whithin the same experiment.

Another way to manage the problem with a determination of the Rydberg constant is to perform two independent absolute frequency measurements (i.e., the measurements with respect to a primary cesium standard). Comparing them afterwards one can determine both the Rydberg constant and the Lamb shift. Combining results from Garching on the $1 s-2 s$ transitions [38] and from Paris on the $2 s-8 s / 8 d / 12 d$ intervals [39], the most accurate optical value of the Lamb shift has been reached (Fig. 12).

Some of the optical experiments were also performed for deuterium (see, e.g., [39]) and that may improve the accuracy in the determination of the Rydberg constant and, thence, indirectly, of the hydrogen Lamb shift.

The recent progress with the visible and ultraviolet optical measurements became in part possible because of use of the frequency comb generator, an advanced technique which allows to compare two optical frequencies to each other and an optical frequency to a microwave frequency [59]. Because of the frequency comb generation, a comparison of two transitions within one experiment gives no advantages anymore. We expect that further progress will be due to independent absolute measurements of different transitions.

The optical experiments delivered data which involve a number of levels $(1 s$, $2 s, 3 s$ etc.). Therefore, a new problem appeared in the increasing number of levels and each of these levels has its own Lamb shift, the value of which should be determined somehow. A large number of quantities to extract from experimental data may reduce advantages of the optical approach. A problem with 
the number of Lamb shifts was solved with the help of the specific difference in Eq. (11) which is found to be of the form [34,35]

$$
\begin{aligned}
\Delta(n)=\frac{\alpha(Z \alpha)^{4}}{\pi} \frac{m_{R}^{3}}{m^{2} c^{2}} \times\{ & -\frac{4}{3} \ln \frac{k_{0}(1 s)}{k_{0}(n s)}\left(1+Z \frac{m}{M}\right)^{2}+C_{\operatorname{Rec}} \frac{Z m}{M} \\
& +(Z \alpha)^{2}\left[A_{61} \ln \frac{1}{(Z \alpha)^{2}}+A_{60}^{V P}+G_{n}^{S E}(Z \alpha)\right. \\
& \left.\left.+\frac{\alpha}{\pi}\left(\ln ^{2} \frac{1}{(Z \alpha)^{2}} B_{62}+\ldots\right)\right]\right\}
\end{aligned}
$$

where the $n$-dependent coefficients $A_{61}, A_{60}^{V P}, C_{\mathrm{Rec}}$ and $B_{62}$ are presented for $n=2$ in Table 2. The coefficients for other $n$ and a table for the function $G^{S E}(Z \alpha)$ can be found in Refs. [35,36]. The uncertainty was also discussed there. A crucial issue is that the difference has a better established status than that for the $1 s$ (or $2 s$ ) Lamb shift. In particular, various QED corrections vanished for the difference, some other are of a reduced numerical value. The finite-nuclear-size contribution, which produces the largest theoretical uncertainty, is cancelled out after combining the $1 s$ and $2 s$ contributions.

To analyze the experimental data, we need to use a certain piece of the bound state QED theory, such as results on the Lamb shift of $2 p_{j}$ states given in Eqs. (13) and (14), on the specific difference $\Delta(n)$ (18) defined in Eq. (11) and a result for the lifetime of the $2 p$ state $(15)$.

Thus, prior to test the QED theory of the hydrogen Lamb shift, some theoretical results have to be applied to extract the experimental values of the Lamb shift from the data of the measurements. Due to that we have to clarify here the word 'theoretical'. To our mind, a value is a theoretical one if it is sensitive to theoretical problems (like, e.g., the problem of the determination of the fundamental constants and phenomenological parameters needed as an input to the calculations and the problem of a proper estimation of uncalculated higher-order QED corrections for the Lamb shift). A calculated value, which is not sensitive to this kind of problems, is not theoretical, but rather a mathematical one.

Rigorously speaking, there is neither pure theoretical value nor experimental value. Theoretical calculations involve experimentally determined values of certain parameters, or even functions, while any measurement needs a theory to interpret measured quantities in terms of collected records of experimental devices.

The main problem of theoretical calculations for the hydrogen Lamb shift is a lack of an accurate determination of the proton charge radius and an insufficient understanding of uncertainty due to the higher-order QED effects. 
The former source of theoretical uncertainty, related to experimental particle physics, dominates. The proton charge distribution does not affect evaluation of experimental data via $E_{L}\left(2 p_{j}\right), \Delta(n)$ and $\tau(2 p)$, while the theoretical uncertainty due to the higher-order QED effects enters the evaluation.

An additional discussion to clarify the problem is needed, since the auxiliary piece of theory needed to evaluate the experimental data for the $1 s$ Lamb shift is related to the Lamb shift theory, which in principle we would like to verify measuring the $1 s$ Lamb shift. A question on self-consistency of such a test of the QED theory of the Lamb shift arises. We emphasize, however, that a crucial point is that the specific difference $\Delta(n)$ for the $n s$ states, which are involved in actual experiments, and the Lamb shift and the lifetime of the $2 p_{j}$ states corresponds to a part of theory which is understood substantially better than theory of the $1 s$ Lamb shift. The higher-order contribution enters auxiliary theoretical expressions for $E_{L}\left(2 p_{j}\right), \Delta(n)$ and $\tau(2 p)$ in an essentially weakened form, since the dominant contributions are proportional to $\delta_{l 0}$.

A successful deduction of the Lamb shift (Fig. 9) in the hydrogen atom provides us with a precision test of bound state QED and offers an opportunity to learn more about the proton size. Bound state QED is quite different from QED for free particles. The bound state problem is complicated by itself even in the case of classical mechanics. The hydrogen atom is the simplest atomic system; however, a theoretical result for the energy levels is expressed as a complicated function (often a perturbation expansion) of a number of small parameters [60] (see review [16] for a collection of theoretical contributions):

- the fine structure constant $\alpha$, which counts the QED loops;

- the Coulomb strength $Z \alpha$;

- the electron-to-proton mass ratio;

- the ratio of the proton size to the Bohr radius.

Indeed, in the hydrogen atom $Z=1$, but it is customary to keep $Z$ even for hydrogen in order to trace out the origin of the corrections, especially because the behavior of expansions in $\alpha$ and $Z \alpha$ differs from each other. In particular, the latter involves large logarithms $\left(\ln (1 / Z \alpha) \simeq 5, \ln ^{2}(1 / Z \alpha) \simeq 24\right.$ and $\ln ^{3}(1 / Z \alpha) \simeq 120$ at $\left.Z=1\right)[60,61]$ and big coefficients. Comparing $\alpha$ and $Z \alpha$ we note that in the relativistic case, when the energy transfer $k_{0}=\Delta E / c$ is of the same order as the momentum transfer $\mathbf{k}$, the parameter is rather $\alpha / \pi$. In the QED loops, such as for the vacuum polarization and the self-energy, the relativistic condition $k_{0} \sim|\mathbf{k}|$ is always a case. Sometimes it is also realized for the exchange loop and in particular in the case of some recoil corrections. However, most of the Coulomb exchanges correspond to another kinematics, which is close to the external field case with a low energy transfer $k_{0} \ll|\mathbf{k}|$ and a real parameter is even not $Z \alpha$ but rather $\pi Z \alpha$ at least for a few first terms of expansion. One of sources of the factor $\pi$ is a fact that the imaginary part 
of logarithm is typically $\pi$, not unity, and with multilogarithmic contributions some factors $\pi$ in non-logarithmic terms must be expected.

Because of a presence of at least three small parameters it is not possible to do any exact calculations and one must use expansions at least in some parameters. The $Z \alpha$ expansion with all its enhancements has a very bad convergency and only in a few cases (some one-loop QED contributions [62] and recoil [63]) calculations exact in $Z \alpha$ have been performed. In such a case the hardest theoretical problem is to estimate systematically all uncalculated terms related to the higher-order corrections of the $Z \alpha$ expansion.

References to recent theoretical results on the $1 s$ Lamb shift theory are collected in Table 3. Calculation of these orders has been completed. Some other corrections are under study (e.g., $\left.\alpha^{3}(Z \alpha)^{5} m c^{2}[78]\right)$.

We note that accuracy of the most recent calculations of the two-loop selfenergy $[67,68]$ is unclear and we ignore it in our analysis. In particular we doubt that the higher order terms may neglected in extrapolation to low $Z$ [68]. On contrary we expect they can produce corrections bigger than claimed in [68] uncertainty of the calculation of the non-logarithmic term. We also do not think that a reference to a similarity with the one-loop correction is enough to claim a $15 \%$ uncertainty for the non-logarithmic term in [68]. The one-loop and two-loop self energies are quite different and in particular they show a very different $n$ dependence when comparing contributions for various $n s$ states. The $n$ dependence is strongly related to splitting of the complete result to partial contributions of specific areas of integration. Besides we were recently informed that the single-logarithm two-loop calculation needs corrections [77].

Table 3

\begin{tabular}{lc|lc}
\hline Correction & Reference(s) & Correction & Reference(s) \\
\hline$\alpha(Z \alpha)^{6} m c^{2}$ & {$[64]$} & $(Z \alpha)^{6} m^{2} c^{2} / M$ & {$[71,63,72]$} \\
$\alpha^{2}(Z \alpha)^{5} m c^{2}$ & {$[65,66]$} & $\alpha(Z \alpha)^{5} m^{2} c^{2} / M$ & {$[73,74,75]$} \\
$\alpha^{2}(Z \alpha)^{6} m c^{2}$ & {$[61,67,68,69]$} & $\alpha(Z \alpha)^{7} m c^{2}$ & {$[62,76]$} \\
$\alpha^{3}(Z \alpha)^{4} m c^{2}$ & {$[70]$} & & \\
\hline
\end{tabular}

References to recent theoretical results on calculations of the $1 s$ Lamb shift.

A pure QED calculation for the hydrogen atom is not enough and before performing any comparison of the QED theory and experiment we need to take into account effects of the internal structure of a proton. 


\section{Lamb shift and effects of the proton charge distribution}

Let us consider a problem related to the distribution of the proton charge in more detail. The leading correction due to the finite size of the proton has a simple expression

$$
\Delta E_{\text {finite size }}(n l)=\frac{2(Z \alpha)^{4} m c^{2}}{3 n^{3}}\left(\frac{m c R_{p}}{\hbar}\right)^{2} \delta_{l 0},
$$

where $R_{p}$ is the mean-squared proton charge radius. However, to reach any accurate numerical value one needs first to determine the value for the proton charge radius with a proper precision. Unfortunately, data available at the moment, if understood literally with their claimed uncertainties, are not reliable (we follow here a consideration in [36]). The dominant contribution to the uncertainty of theory of the Lamb shift of the $1 s$ state is due to the contribution (19) [36]. It appears that currently the most accurate value of $R_{p}$ can be obtained by reading a comparison of the experimental value of the Lamb shift and theory in a reversed way: not testing the bound state QED, but determining the proton charge radius [36]

$$
R_{p}(\text { Lamb shift }) \simeq 0.89(2) \mathrm{fm} .
$$

The prediction for the theoretical value of the Lamb shift strongly depends on what value for the proton charge radius is accepted. A crucial level of accuracy of the radius is $1 \%$, which is related to the case when all three uncertainties (experimental, QED and due to the proton radius) are approximately the same. Two values were under a long discussion inside the atomic and QED community:

$$
R_{p}(\text { Stanford })=0.805(11) \mathrm{fm}, \quad[79]
$$

and

$$
R_{p}(\text { Mainz })=0.862(12) \mathrm{fm}, \quad[80] .
$$

More data for the proton radius are collected in Fig. 13.

To discuss the scatter of the data, let us look at the most important data on the electron-proton elastic scattering presented in Fig. 14. Even from the first glance one can realize that the Mainz data are

- presented by a larger number of points;

- more accurate; 


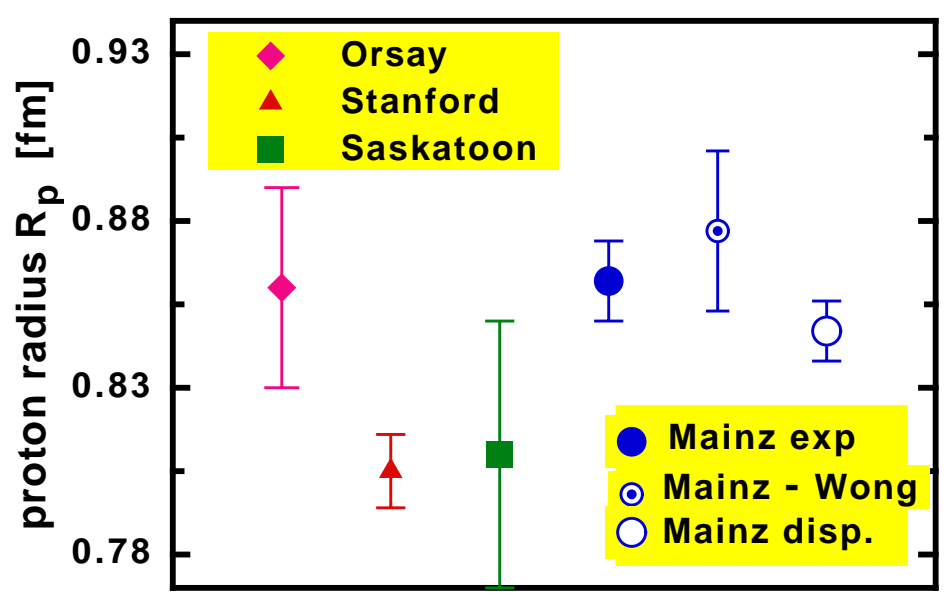

Fig. 13. Proton charge radius determined from the scattering experiments. The presented results are phenomenologically extracted from the scattering at Orsay [81], Stanford [79], Saskatoon [82] and Mainz [80] or found with more sophisticated analysis from Mainz data by Wong [83] and by Mainz theoretical group from a multi-parameter dispersion fit of all available data [84].

- obtained for a more broad range of the momentum transfer including the lowest momentum transfer ever achieved.

The latter is important since the easiest way to extract the proton charge radius from the data is to extrapolate to zero momentum transfer

\section{form factor $G\left(q^{2}\right)$}

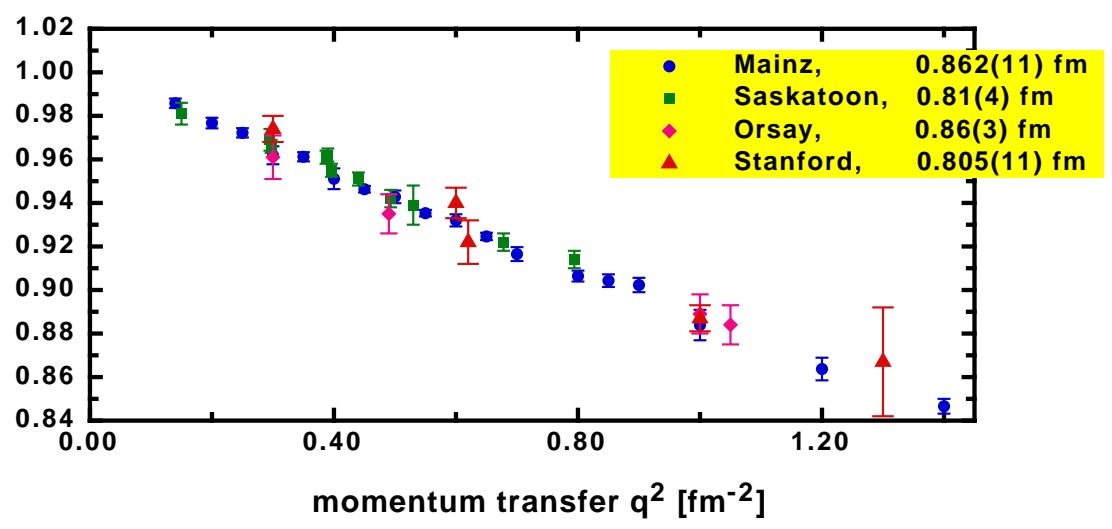

Fig. 14. Data for the electric form factor of the proton from the electron-proton elastic scattering experiments performed at Orsay [81], Stanford [79], Saskatoon [82] and Mainz [80].

$$
\begin{aligned}
G\left(q^{2}\right) & =a_{0}+a_{1} q^{2}+a_{2} q^{4}+\ldots \\
& =a_{0} \cdot\left(1-\frac{R_{p}^{2}}{6} \mathbf{q}^{2}+\ldots\right)
\end{aligned}
$$


where $q^{2}=-\mathbf{q}^{2}$ is related to the three-dimensional momentum transfer. There is no chance for other data in Fig. 14 to compete with the Mainz results $[80]^{3}$. The Mainz experiment is the most appropriate to precisely determine the proton radius since it contains more points at lower momentum transfer and with a higher precision than the rest of the data. Due to this any compilation containing the Mainz data has to lead to a result close to that from the Mainz data only, because the Mainz scattering points must be statistically responsible for the final result. In particular, we believe the dispersion analysis performed by Mainz theorists [84] led to such a result ${ }^{4}$

$$
R_{p}(\operatorname{disp})=0.847(9) \mathrm{fm}
$$

which, however, differs from the empirical value of $R_{p}=0.862(12)$ fm from Eq. (22) [80].

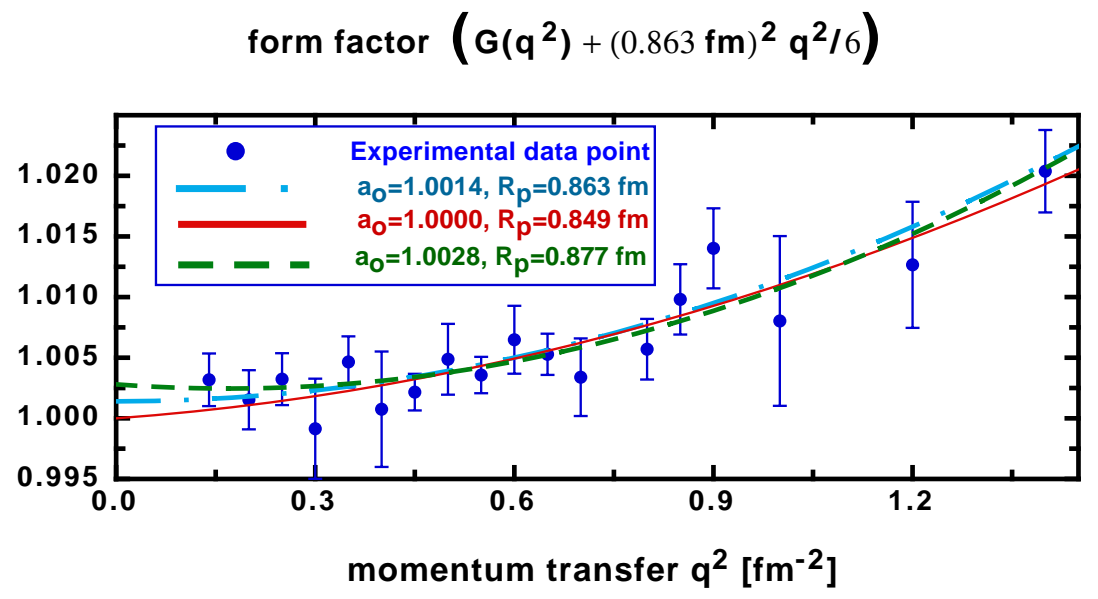

Fig. 15. Fitting the electric form factor of the proton from the Mainz experimental data [80]. Since details of fitting by Wong [83] and a particular result on the coefficient $a_{2}$ are not available, we present here similar fits from [36].

One of the problems in evaluating the data is their normalization and actually a different treatment of this problem is in part responsible for the discrepancy between results from Eqs. (22) and (24). One can write a low-momentum expansion of the form factor from Eq. (23) and expect that $G(0)=1$. However, a real situation is more complicated and somewhat confusing. The confusion arises from use of the words 'form factor'. The definition of the form factor is

3 I have learned that from a similar picture presented by Malcolm Boshier for ICAP 1996 (see Fig. 5 in [85]).

4 Unfortunately, the authors of [84] are neither able to explain which part of data statistically dominates (if any) nor to prove properly the estimation of the uncertainty assigned by them to the nucleon radii since the radii were not main objectives of their fitting procedure and appeared in the very end of the evaluation as a complicated function of numerous correlated fitting parameters. 
clearly understood theoretically, however, the true form factor cannot be measured accurately. In the case of scattering one has to deal with the Rosenbluth formula which describes the cross section. To make an absolute measurement of the cross section, one needs to measure parameters of the incoming electron beam with a high accuracy, as well as determine the efficiency of the detectors etc. For a relative measurement one has only to ensure that the details of the beam and detections are the same during the entire experiment. The normalization is very complicated and sometimes involves much more efforts than the relative measurements. However, the normalization measurement used to be not enough accurate. In particular, in the Mainz case the uncertainty is about $0.5 \%$. That means that a value of $G\left(q^{2}\right)$ tabulated from the scattering data, as being the form factor, actually differs from the true form factor with some normalization factor, which is consistent with but not equal to unity.

There is an alternative wording to explain the situation and it may be helpful for some readers. When the experimentally determined form factor is presented properly it contains a statistical error (mainly related to relative measurements) and a systematic error (mainly due to the normalization). The measured form factor in such a case directly related to the 'theoretical form factor' and its value at zero momentum transfer is equal to unity. However, as we mentioned the systematic error is often higher than the statistical error (at least eventually, when the statistical error for the fitting parameters is suppressed by the number of data points). That means that if we at the first approximation would ignore the systematic error we still should be able to obtain a result almost without loosing in accurate. If we drop the systematic error, the central values of the form factor remain the same, however, it physical meaning is changed drastically. The measured value $G_{\text {meas }}(q)$ can be presented as

$$
G_{\text {meas }}(q)=G_{\text {meas }}^{\prime}(q) \cdot(1 \pm \delta)
$$

where $\delta$ is the systematic error of the normalization. While the measured form factor is defined in such a way that $G_{\text {meas }}(0)=1$; the value with only a statistical uncertainly satisfies a condition $G_{\text {meas }}^{\prime}(0)=1 /(1 \pm \delta)$, i.e., it must be consistent with unity at zero momentum transfer, but not just equal to it. That is to what we refer above as to a conventional normalization.

The confusion comes in part from the common attempt to normalize data as close to the form factor as possible to make their physical meaning more clear. However, from a pure practical point of view that has no sense. The lack of accurate data on the normalization makes $G(0)$ consistent with unity within the experimental uncertainty of the normalization, which is nearly always larger than the uncertainty of relative measurements. The normalization uncertainty is a systematic error, while the data are often tabulated with only statistical component of the uncertainty. 
A few different fits were performed by Wong [83] (see Fig. 15). Two of them were related to the normalization used in $[80]$ and $[84]^{5}$ and the achieved results were close to published there. A fit with a free value of $a_{0}$ led to a larger uncertainty $R_{p}=0.88(2) \mathrm{fm}$ (see the Wong-Mainz value in Fig. 13). To our mind, even this result must be treated with a certain caution. It is necessary to take into account some higher-order corrections and that is not possible because of the absence of any complete description of the experiment [80]. Our conservative estimation of the theoretical uncertainty in Fig. 9 is related to $[36,60]$

$$
R_{p}(\text { conservative })=0.88(3) \mathrm{fm} .
$$

The present status of the Lamb shift of the $1 s(2 s)$ state is that

- the QED computation uncertainty is about $2 \mathrm{ppm}$,

- the measurement uncertainty of the grand average value is $3 \mathrm{ppm}$,

- while the uncertainty due to the proton size is about $10 \mathrm{ppm}$.

Some other analysis of the proton radius can be found in [86]. The result $R_{p}=0.880(15) \mathrm{fm}$ is consistent with (25). It is likely that a proper normalization still is the most important source of correcting the central value of $R_{p}$ and the increase of its uncertainty. A very different treatment by Sick [87] which takes into account Coulomb effects and various systematic sources led to a result $R_{p}=0.895(18) \mathrm{fm}$ also consistent with our value in (25). Our uncertainty is somewhat bigger than that of $[83,86,87]$ because we consider possible systematic errors due to higher order QED corrections [36] to scattering which are beyond evaluation in [80].

Improvement of the determination of the proton charge radius is expected from a study of the Lamb shift in muonic hydrogen. As one can see from discussion in Sect. 2, the line width scales linearly with the mass of the orbiting particle, while the finite-nuclear-size contribution is proportional to $\mathrm{m}^{3}$ and that offers a good chance for an accurate determination of the proton charge radius. The level scheme of the experiment which is in progress at Paul Scherrer Institut [88] is presented in Fig. 16. The metastable $2 s$ state is to be excited by the laser light to the $2 p$ state which then decays to the ground state producing an $\mathrm{X}$-ray Lyman- $\alpha$ photon. The intensity of the X-ray fluorescence is studied as a function of the laser frequency.

The problem of the nuclear size is not only a problem of the hydrogen Lamb shift: a similar situation arises with the helium-4 ion Lamb shift, where uncertainties resulting from the QED computation and the nuclear size are about

5 The paper [83] was published prior to [84], however, it was indeed natural to anticipate the use of the 'theoretical' normalization $G(0)=1$. 


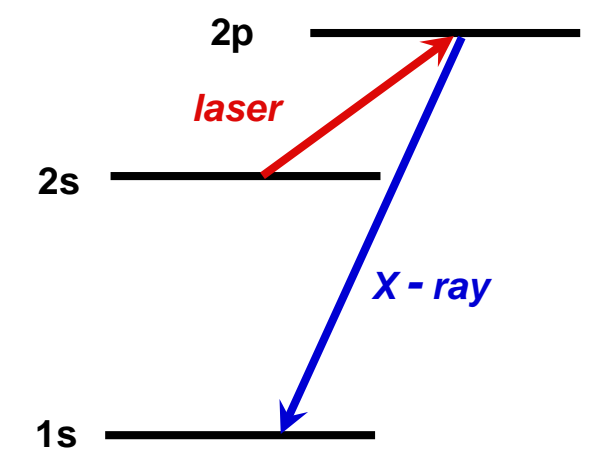

Fig. 16. Level scheme of the PSI experiment on the Lamb shift in a muonic hydrogen [88] (not to scale). The hyperfine structure is not shown.

the same. Theory is in agreement with experiment [89] and the leading theoretical uncertainty is related to the nuclear size. The theoretical prediction for the Lamb shift in deuterium has a bigger uncertainty because of the nuclear polarizability effects [90]. The latter are significant since a deuteron is known as a very loosely nuclear bound system. For more detail on the nuclear physics of light nuclei and their effects on atomic energy levels, see [91].

\section{Hyperfine splitting in light hydrogen-like atoms and the nuclear structure}

A similar problem of significant interference of the nuclear structure and QED effects exists for the hyperfine structure (HFS) in the hydrogen atom and actually the case of the hyperfine structure is in a sense even much less favorable for the QED tests. The hyperfine interval in the ground state was for a while the most accurately measured quantity being known with an uncertainty at the level of a part in $10^{12}$. The results of the most accurate experiments are summarized in Fig. 17. It so happened that most results were published in metrological journals and were missing in various 'physical' compilations. This figure includes only results published in refereed journals, but not those from conference proceedings. We also note that the most frequently quoted result $[102]$

$$
f_{\mathrm{HFS}}(1 s)=1420405.751767(1) \mathrm{kHz}
$$

was later corrected [94] and the uncertainty was increased by a factor of 3 .

We summarize in Tables 4 all accurate data available for the hyperfine intervals of the $1 s$ and $2 s$ states in conventional light hydrogen-like atoms (hydrogen, deuterium and tritium and in the helium-3 ion). Pure leptonic atoms are considered separately in Sects. 8 and 9 . 


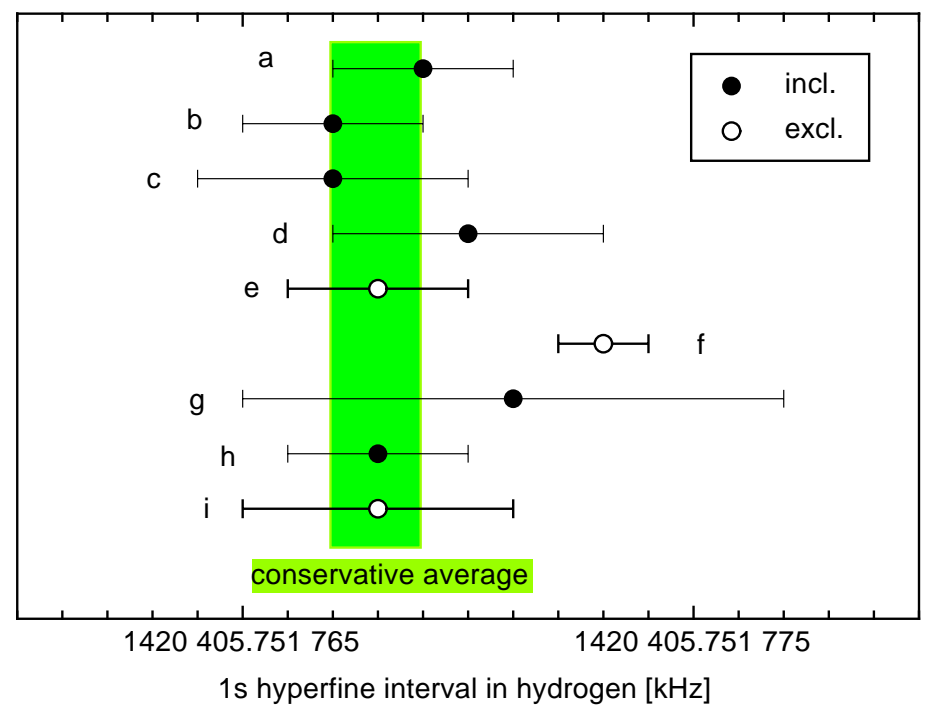

Fig. 17. Measurement of the $1 s$ hyperfine splitting in the hydrogen atom. The references are: $a-[92]$ (experiment \#1 with the wall-shift determined in [93]), $b-$ [92] (experiment \#2), $c-[94], d-[95], e-[96], f-[97], g-[98], h-[99], i-[100]$. The conservative average, $1420405.751768(1) \mathrm{kHz}$, is presented according to [101]. It is an average over the results denoted by filled circles, while the results presented by open circles are excluded from evaluation (see [101] for detail).

A value of the hyperfine interval in the $n s$ state is determined in the leading order by the so-called Fermi energy $E_{F}$ as following

$$
\begin{aligned}
E_{n s}(\text { leading }) & =E_{F} \cdot \frac{1+a_{e}}{n^{3}} \\
& =-\frac{8 \pi \alpha}{3} \frac{\hbar^{3}}{m_{e}^{2} c} \frac{\left\langle\boldsymbol{\mu}_{e} \cdot \boldsymbol{\mu}_{\mathrm{Nucl}}\right\rangle}{\mu_{B}^{2}}\left|\Psi_{n s}(\mathbf{r}=0)\right|^{2}
\end{aligned}
$$

Here $\mu_{e}$ and $\mu_{\text {Nucl }}$ stand for the magnetic moment of a free electron and the nucleus, respectively, and

$$
\left|\Psi_{n l}(\mathbf{r}=0)\right|^{2}=\frac{1}{\pi}\left(\frac{Z \alpha m_{R} c}{n \hbar}\right)^{3} \delta_{l 0}
$$

is a squared value of the non-relativistic wave function at the origin. Note, that we have included a contribution of the anomalous magnetic moment of the electron into the leading term $E_{n s}$ (leading), but not into the definition of $E_{F}$.

The most important corrections to (26) are due to relativistic effects, boundstate quantum electrodynamics and due to the nuclear structure. In the case of a point-like infinitely heavy nucleus, i.e., when only the relativistic and bound 


\begin{tabular}{|c|c|c|c|c|}
\hline $\begin{array}{l}\text { Atom, } \\
\text { state }\end{array}$ & $\begin{array}{c}E_{\mathrm{HFS}}(\exp ) \\
{[\mathrm{kHz}]}\end{array}$ & Ref. & $\begin{array}{c}E_{\mathrm{HFS}}(\mathrm{QED}) \\
{[\mathrm{kHz}]}\end{array}$ & $\begin{array}{c}\Delta E \\
{[\mathrm{ppm}]}\end{array}$ \\
\hline Hydrogen, $1 s$ & $1420405.751768(1)$ & {$[92,93,94,95,98,99,101]$} & 1420452 & -33 \\
\hline Deuterium, $1 \mathrm{~s}$ & $327384.352522(2)$ & {$[103]$} & 327339 & 138 \\
\hline Tritium, $1 s$ & $1516701.470773(8)$ & {$[104]$} & 1516760 & -38 \\
\hline${ }^{3} \mathrm{He}^{+}$ion, $1 s$ & - $8665649.867(10)$ & {$[105]$} & -8667494 & -213 \\
\hline Hydrogen, $2 s$ & $177556.860(16)$ & {$[106]$} & 177562.7 & -32 \\
\hline Hydrogen, $2 s$ & $177556.785(29)$ & {$[107]$} & & -33 \\
\hline Hydrogen, $2 s$ & $177556.860(50)$ & {$[108]$} & & -32 \\
\hline Deuterium, $2 s$ & $40924.454(7)$ & {$[109]$} & 40918.82 & 137 \\
\hline Deuterium, $2 s$ & $40924.439(20)$ & {$[110]$} & & 137 \\
\hline${ }^{3} \mathrm{He}^{+}$ion, $2 s$ & - $1083354.9807(88)$ & {$[111]$} & - 1083585.7 & -213 \\
\hline${ }^{3} \mathrm{He}^{+}$ion, $2 s$ & - $1083354.99(20)$ & {$[112]$} & & -213 \\
\hline
\end{tabular}

Table 4

Hyperfine structure in light hydrogen-like atoms. The numerical results are presented for the frequency $E / h$. The difference $(\Delta E)$ between the actual value $\left(E_{\mathrm{HFS}}(\exp )\right)$ and a result of the pure QED calculation $\left(E_{\mathrm{HFS}}(\mathrm{QED})\right)$ is caused by the nuclear structure. The negative sign for the ${ }^{3} \mathrm{He}^{+}$ion reflects the fact that the nuclear magnetic moment is negative, i.e., in contrast to other nuclei in the Table, its direction is antiparallel to the nuclear spin.

state QED contributions are taken into account, the correction to (26) is of the form

$$
\begin{aligned}
\Delta E_{1 s}(\mathrm{QED})=E_{F} & \times\left\{\frac{3}{2}(Z \alpha)^{2}+\alpha(Z \alpha)\left(\ln 2-\frac{5}{2}\right)\right. \\
& +\frac{\alpha(Z \alpha)^{2}}{\pi}\left[-\frac{2}{3} \ln \frac{1}{(Z \alpha)^{2}}\left(\ln \frac{1}{(Z \alpha)^{2}}\right.\right. \\
& \left.+4 \ln 2-\frac{281}{240}\right)+17.122339 \ldots \\
& \left.\left.-\frac{8}{15} \ln 2+\frac{34}{225}\right]+0.7718(4) \frac{\alpha^{2}(Z \alpha)}{\pi}\right\} .
\end{aligned}
$$

This term is in fact smaller than the nuclear-structure correction (see Table 5). The contributions induced by the nuclear structure are determined there by subtracting the results of the pure QED calculations

$$
E_{\mathrm{HFS}}(\mathrm{QED})=E_{n s}(\text { leading })+\Delta E_{n s}(\mathrm{QED})
$$


from the actual values of the hyperfine intervals (see Table 4)

$$
E_{\mathrm{HFS}}(\mathrm{Nucl})=E_{\mathrm{HFS}}(\exp )-E_{\mathrm{HFS}}(\mathrm{QED})
$$

The result for the $1 s$ state is presented in Eq. (28) and the $2 s$ result is of a similar form and discussed in detail in the next section. We mainly follow here [113], but the QED helium result in Table 4 and related data in Tables 5 and 6 are corrected as explained in [114] and [115,116] (cf. [113]).

Table 5

\begin{tabular}{lcccc}
\hline Atom & $\begin{array}{c}\Delta E(\mathrm{QED}) \\
{[\mathrm{ppm}]}\end{array}$ & $\begin{array}{c}(Z \alpha)^{2} E_{F} \\
{[\mathrm{ppm}]}\end{array}$ & $\begin{array}{c}\alpha(Z \alpha) E_{F} \\
{[\mathrm{ppm}]}\end{array}$ & $\begin{array}{c}\Delta E(\mathrm{Nucl}) \\
{[\mathrm{ppm}]}\end{array}$ \\
\hline Hydrogen & -23 & 80 & -96 & -33 \\
Deuterium & -23 & 80 & -96 & 138 \\
Tritium & -23 & 80 & -96 & -38 \\
${ }^{3} \mathrm{He}^{+}$ion & 108 & 319 & -192 & -213 \\
\hline
\end{tabular}

A comparison of the bound QED and nuclear-structure corrections to the $1 s$ hyperfine interval. The QED term $\Delta E(\mathrm{QED})$ defined in (28) contains only the boundelectron effects while the anomalous magnetic of free electron $a_{e}=\alpha / 2 \pi+\ldots \simeq$ $1.159652 \ldots \times 10^{-3}$ is excluded. Additionally, to the whole QED correction we also present separately two major contributions into it: the leading relativistic term in order $(Z \alpha)^{2} E_{F}$ and the leading bound state QED correction in order $\alpha(Z \alpha) E_{F}$. The nuclear contribution $\Delta E(\mathrm{Nucl})$ has been found via a comparison of the experimental results with the pure QED values (see Table 4).

There are three kinds of corrections which involve the nuclear-structure effects.

- The dominant contribution for the hydrogen HFS interval is due to a distribution of the charge and magnetic moment inside the nucleus (so-called Zemach correction)

$$
\Delta E(\text { Zemach })=\frac{2 Z \alpha}{\pi^{2}} E_{F} \times m c \int \frac{d^{3} \mathbf{q}}{\mathbf{q}^{4}}\left[\frac{G_{E}\left(-\mathbf{q}^{2}\right) G_{M}\left(-\mathbf{q}^{2}\right)}{1+\kappa}-1\right]
$$

where the electric/magnetic form factor $G_{E / M}\left(-\mathbf{q}^{2}\right)$ is a Fourier transform of the space distribution of the proton electric charge/magnetic moment $\rho_{E / M}(\mathbf{r})$ and $\kappa$ is the proton anomalous magnetic moment ${ }^{6}$. In the coordi-

${ }^{6}$ It is necessary to mention that a straightforward consideration of the form factor as a Fourier transform of the space distribution of the related value (such as the electric charge) is a transparent but rough approximation, which fails for the relativistic momentum transfer (see, e.g., [117]). 
nate space the equation takes the form

$$
\Delta E(\text { Zemach })=-\frac{2(Z \alpha) m c}{\hbar} E_{F} \times \int d^{3} \mathbf{r} d^{3} \mathbf{r}^{\prime} \rho_{E}(\mathbf{r}) \rho_{M}\left(\mathbf{r}^{\prime}\right)\left|\mathbf{r}-\mathbf{r}^{\prime}\right|
$$

For light atoms the uncertainty in (29) comes from the distribution of the magnetic moment which involves the magnetic form factor $G_{M}\left(-\mathbf{q}^{2}\right)$ at low momentum transfer, which is difficult to study experimentally in such kinematic region (in contrast to the electric form factor $G_{E}$ ).

- A different effect dominates for a deuteron, which is a loosely bound system and the electron can see it not only as the whole but can also recognize its composites, namely a proton and a neutron. This is a nuclear polarizability contribution.

- A theory of a point-like particle with a non-vanishing value of the anomalous magnetic moment is inconsistent ${ }^{7}$. In particular, calculating recoil corrections to the hyperfine structure in atoms with such a nucleus, certain divergencies have to appear at high momentum. Those are not ultraviolet QED divergencies. They are related to an inconsistency of the consideration of the operators of the non-relativistic expansion as fundamental operators. The operator $\sigma_{\mu \nu} k_{\mu} / M c$ is such an operator and it gives reasonable results only when $k / M c \ll 1$. A complete theory with the finite nuclear size removes all divergencies of this kind and thus the nuclear size serves as an effective cut-off for them entering the equations originally designed for a point-like particle. Since the divergence in the related recoil contribution to the HFS interval is logarithmic, the recoil effects only slightly depend on this cut-off and through it on detail of the nuclear structure.

The magnitude of the nuclear corrections to the hyperfine interval in light hydrogen-like atoms lies at the level from 30 to $200 \mathrm{ppm}$ (depending on the atom) and theoretical understanding of such effects is unfortunately by far not sufficient $[36,118,113]$. The correction is compatible with the bound-state QED term (note that the anomalous magnetic moment is related to the free QED consideration and it is included into the leading term, but not into the QED correction). The results for the $1 s$ state are summarized in Table 5 (see

\footnotetext{
7 We need to mention a certain inconsistency in the terminology related to the leptons. They are referred as "point-like" since all effects of the distribution of the electric charge and the magnetic moment can be derived from QED. However, they "have a non-vanishing anomalous magnetic moment" which comes from the same QED effects. Indeed, both effects are to be considered on the same ground. The inconsistency historically comes from the fact that the anomalous magnetic moments was first discovered experimentally and later explained, while the "electron charge radius" is hardly to be seen in a straightforward experiment but can be easily considered theoretically. The Lamb shift is a result of the electron charge distribution related to the electron structure, however, it is understood only through the QED interpretation. So the internal electron structure effects look rather as a theoretical construction, while the anomalous magnetic moment is a direct experimental fact.
} 
Ref. [113] for detail). The results for the $2 s$ state are slightly different for the relative value of the QED contribution. A certain progress [119] is on the way, however, it should more contribute to nuclear physics since a gap between accuracy of a pure QED theory and that of the nuclear effects is of a few orders of magnitude and cannot be avoided.

From Table 4 one can learn that in the relative units the effects of the nuclear structure are nearly the same for the $1 s$ and $2 s$ intervals (33 ppm for hydrogen, $138 \mathrm{ppm}$ for deuterium and $213 \mathrm{ppm}$ for helium-3 ion). A reason for that is the factorized form of the nuclear contributions in the leading approximation (cf. (19))

$$
\Delta E(\mathrm{Nucl})=A(\mathrm{Nucl}) \times\left|\Psi_{n l}(\mathbf{r}=0)\right|^{2}
$$

In other words, the correction is a product of the nuclear-structure parameter $A(\mathrm{Nucl})$ and the squared wave function at the origin (27), which is a result of a pure atomic problem (a nonrelativistic electron bound by the Coulomb field). The nuclear parameter $A(\mathrm{Nucl})$ depends on the nucleus (a proton, a deuteron etc.) and on the effect under study (the hyperfine structure, the Lamb shift), but does not depend on the atomic state. We remind, that, similarly, the leading term for the HFS interval for an $n s$ state in Eq. (26) is also proportional to squared wave function at the origin $\left|\Psi_{n l}(\mathbf{r}=0)\right|^{2}$ and thus a fractional value of the nuclear contribution is kept $n$ independent in the leading approximation.

Two parameters in the hydrogen-like wave function (27) can be varied for the same nuclei:

- the reduced mass of a bound particle for conventional (electronic) atoms $\left(m_{R} \simeq m_{e}\right)$ and muonic atoms $\left(m_{R} \simeq m_{\mu}\right)$;

- the principle quantum number $n=1,2$ for the $1 s$ and $2 s$ states.

We note that the nuclear charge $Z$ is indeed fixed if we like to deal with the same parameter $A(\mathrm{Nucl})$ (i.e., with the same nucleus).

The former option was already mentioned when we considered the determination of the proton charge radius via the measurement of the Lamb shift in muonic hydrogen [88]. In the next section we consider the latter option, a comparison of the $1 s$ and $2 s$ hyperfine intervals in hydrogen, deuterium and the ${ }^{3} \mathrm{He}^{+}$ion. The $1 s$ state is the ground state and the $2 s$ state is metastable. Other levels are not accessible for the precision experiments being broad because of their radiative decay. 


\section{Hyperfine structure, free of the nuclear effects: comparison of the HFS intervals for the $1 s$ and $2 s$ states}

As we have seen in the previous section, both the $1 s$ and $2 s$ hyperfine intervals in various light hydrogen-like atoms are measured more accurately than theory can predict. Theory, although limited by the uncertainty in the calculation of the nuclear effects, can still proceed to a higher accuracy dealing with a specific difference

$$
D_{21}=8 \cdot E_{\mathrm{HFS}}(2 s)-E_{\mathrm{HFS}}(1 s)
$$

where any contribution (and, in particular, related to the leading nuclear effects) which has the form of Eq. (31) should vanish. Removing the main obstacle on the way of theory, we may study effects related to higher-order QED contributions.

The difference (32) was studied theoretically in several papers long time ago $[120,121,122,123]$. A result of the former study reads

$$
\begin{aligned}
D_{21}(\mathrm{QED} 3) & =(Z \alpha)^{2} E_{F} \times\left\{\frac{5}{8}+\frac{\alpha}{\pi}\left[\left(\frac{16}{3} \ln 2-7\right) \ln (Z \alpha)-5.55155 \ldots\right]\right. \\
& \left.+\frac{m}{M}\left[\left(\frac{\ln 2}{2}-\frac{7}{32}\right)\left(1-\frac{1}{\eta}\right)-\frac{9}{8}-\left(\frac{145}{128}-\frac{7}{8} \ln 2\right) \eta\right]\right\}(33)
\end{aligned}
$$

where

$$
\eta=\frac{\mu}{\mu_{B}} \frac{M}{m} \frac{1}{Z I} .
$$

We remind here about a difference in the notation in particle and nuclear physics. If the $g$ factor of a nucleus would be defined in the particle notation, one has to arrive at $\eta$.

The HFS difference $D_{21}$ is similar to $\Delta(n)$ in Eq. 11, however, there are a number of very different features. The HFS difference is designed to be compared with experimental data and it is of interest only for the $1 s$ and $2 s$ states. The Lamb difference $\Delta(n)$ is to be used for the interpretation of the data and those involves various $n$, still $n=2$ is the most important for application case. The leading term $E_{n s}$ (leading), the leading free QED (due to the anomalous magnetic moment of the electron) and the leading bound state QED corrections cancel for the HFS difference. On the contrary, the leading contribution to the Lamb shift does not cancel for $\Delta(n)$. Still, there is a similarity in calculations of various bound state $\mathrm{QED}$ contributions. 
Table 6

\begin{tabular}{lccc}
\hline Contribution to HFS in & $\begin{array}{c}\text { Hydrogen } \\
{[\mathrm{kHz}]}\end{array}$ & $\begin{array}{c}\text { Deuterium } \\
{[\mathrm{kHz}]}\end{array}$ & $\begin{array}{c}{ }^{3} \mathrm{He}^{+} \text {ion } \\
{[\mathrm{kHz}]}\end{array}$ \\
\hline$D_{21}(\mathrm{QED} 3)$ & 48.937 & 11.3056 & -1189.253 \\
$D_{21}(\mathrm{QED} 4)$ & $0.018(5)$ & $0.0044(10)$ & $-1.13(14)$ \\
$D_{21}(\mathrm{Nucl})$ & -0.002 & $0.0026(2)$ & $0.307(35)$ \\
\hline$D_{21}($ theo $)$ & $48.953(5)$ & $11.3125(10)$ & $-1190.08(15)$ \\
\hline
\end{tabular}

Theory of the specific difference $D_{21}=8 E_{\mathrm{HFS}}(2 s)-E_{\mathrm{HFS}}(1 s)$ in light hydrogen-like atoms (see $[115,116,113,114]$ for detail). The numerical results are presented for the related frequency $D_{21} / h$.

Recent studies of $D_{21}$ [124] showed that certain higher-order QED and nuclear corrections have to be taken into account for a proper comparison of theory and experiment. The theory has been essentially improved $[113,125]$ and it is summarized in Table 6. The QED corrections up to the third order $\left(D_{21}(\mathrm{QED} 3)\right)$ and the fourth-order contribution of the order $(Z \alpha)^{4}$ (in units of the $1 s$ hyperfine interval) have been known for a while $[121,122,123,120]$. The new issue here is a complete result for the fourth-order QED contributions $\left(D_{21}(\mathrm{QED} 4)\right)$ and nuclear corrections $\left(D_{21}(\mathrm{nucl})\right)$.

The QED contributions in the fourth order are summarized in Table 7. The new QED contributions are of the order $\alpha(Z \alpha)^{3}, \alpha^{2}(Z \alpha)^{4}, \alpha(Z \alpha)^{2} m / M$ and $(Z \alpha)^{3} \mathrm{~m} / M$. The difference with [113] is a more realistic estimation of the $\alpha(Z \alpha)^{3}$ uncertainty $[115,116]$. In the former paper we accepted result from $[125]$.

Both results for $\alpha(Z \alpha)^{3}$ at $Z=1,2$, in [115,116] and [125], are based on extrapolations of numerical data obtained in [125] for higher values of $Z$. The procedure leads to a calculation of central values and its uncertainty. While the calculation of the central value is approximately the same, the uncertainty is quite different. Systematic uncertainty of the fitting pro cedure is due to a possible perturbation of the fit by certain higher-order terms. As we shown in $[115,116]$, one should expect certain relatively large numerical coefficients for these terms and that increases the uncertainty.

The higher-order nuclear effects survive the cancellation of the leading term but they are substantially smaller than the leading term and can be successfully calculated phenomenologically [113]

$$
\begin{aligned}
D_{21} & =\left(\ln 2+\frac{3}{16}\right) \cdot(Z \alpha)^{2} \cdot E_{\mathrm{hfs}}^{\mathrm{Nucl}}(1 s) \\
& +\left(2-\frac{4}{3} \ln 2\right) \cdot(Z \alpha)^{2}\left(\frac{m c R_{E}}{\hbar}\right)^{2} E_{F}-\frac{1}{4} \cdot(Z \alpha)^{2}\left(\frac{m c R_{M}}{\hbar}\right)^{2} E_{F} .
\end{aligned}
$$




\begin{tabular}{lcccc}
\hline Contribution & $\begin{array}{c}\mathrm{H} \\
{[\mathrm{kHz}]}\end{array}$ & $\begin{array}{c}\mathrm{D} \\
{[\mathrm{kHz}]}\end{array}$ & $\begin{array}{c}{ }^{3} \mathrm{He}^{+} \\
{[\mathrm{kHz}]}\end{array}$ & Ref. \\
\hline$(Z \alpha)^{4} E_{F}$ & 0.0056 & 0.0013 & -0.543 & {$[120]$} \\
$\alpha^{2}(Z \alpha)^{2} E_{F}$ & $0.0033(16)$ & $0.0008(4)$ & $-0.069(35)$ & {$[124,113]$} \\
$\alpha(Z \alpha)^{2} \frac{m}{M} E_{F}$ & $-0.0031(15)$ & $-0.0004(2)$ & $0.022(11)$ & {$[124,113]$} \\
$\alpha(Z \alpha)^{3} E_{F}(\mathrm{SE})$ & $0.008(4)$ & $0.0019(9)$ & $-0.39(14)$ & {$[115,116,125]$} \\
$\alpha(Z \alpha)^{3} E_{F}(\mathrm{VP})$ & 0.0030 & 0.0007 & -0.145 & {$[124,113]$} \\
$(Z \alpha)^{3} \frac{m}{M} E_{F}$ & $0.0005(5)$ & 0.0001 & $-0.007(10)$ & {$[124,113]$} \\
\hline$D_{21}(\mathrm{QED} 4)$ & $0.018(5)$ & $0.0044(10)$ & $-1.13(14)$ & \\
\hline
\end{tabular}

Table 7

The fourth order QED contributions to the $D_{21}$ in hydrogen, deuterium and helium3 ion (see $[115,116,113]$ for detail).

The results for the light atoms are summarized in Table 6. A value for the nuclear correction in the helium-3 ion is slightly different here from the result in [113] due to different nuclear parameters we used (see Table 8).

\begin{tabular}{lccccccc}
\hline Atom & $Z$ & $I$ & $M / m$ & $\begin{array}{c}\mu / \mu_{B} \\
{\left[10^{-3}\right]}\end{array}$ & $\begin{array}{c}E_{F} \\
{[\mathrm{kHz}]}\end{array}$ & $\begin{array}{c}R_{E} \\
{[\mathrm{fm}]}\end{array}$ & $\begin{array}{c}R_{M} \\
{[\mathrm{fm}]}\end{array}$ \\
\hline Hydrogen & 1 & $1 / 2$ & 1836.153 & 1.521032 & 1418840 & $0.88(3)$ & $0.86(4)$ \\
Deuterium & 1 & 1 & 3670.483 & 0.4669755 & 326968 & $2.13(1)$ & $2.07(2)$ \\
Tritium & 1 & $1 / 2$ & 5496.922 & 1.6223936 & 1515038 & $1.76(9)$ & $1.84(18)$ \\
${ }^{3} \mathrm{He}^{+}$ & 2 & $1 / 2$ & 5495.885 & -1.158741 & -8656527 & $1.96(3)$ & $1.97(15)$ \\
\hline
\end{tabular}

Table 8

Parameters for the calculations of the HFS interval in hydrogen, deuterium and helium-3 ion. The proton charge radius is presented according to Ref. [36] while the magnetic radius is from [126]. The radii for other nuclei are taken from [127], while the masses and magnetic moments are from $[23,128,129,115]$. One should use the values for the radii with caution. The results in $[127,87,126]$ are obtained from the scattering data as they were published. However, in a number of papers published decades ago certain radiative corrections were not taken into account (cf. our analysis in [36]) and even a perfect evaluation with uncorrected data underestimates the uncertainty.

For all atoms in Table 4 the hyperfine splitting in the ground state was measured more accurately than for the $2 s$ state. All experimental results but two were obtained by a direct measurement of the microwave transitions for the $1 \mathrm{~s}$ and $2 s$ hyperfine intervals. However, two of the most recent results obtained for hydrogen [106,115] and deuterium [109,115] have been achieved by means of laser spectroscopy and the measured transitions lie in the ultraviolet range [106]. The hydrogen level scheme is depicted in Fig. 18. The measured transitions were the singlet-singlet $(F=0)$ and triplet-triplet $(F=1)$ two-photon 


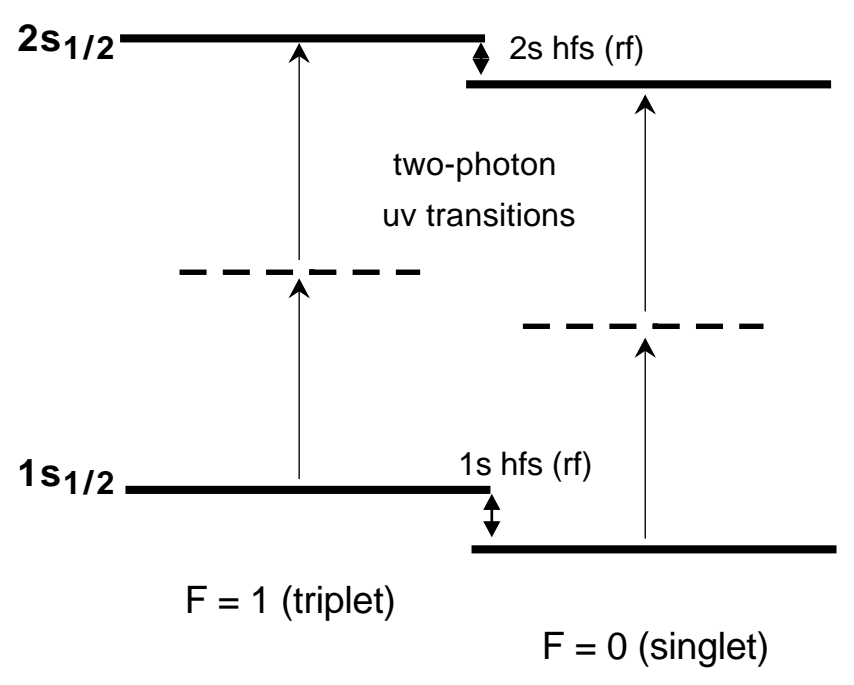

Fig. 18. Level scheme for the optical measurement of the $2 s$ hyperfine structure in the hydrogen atom (not to scale) [106,115]. The label $r f$ stands here for radiofrequency intervals, while $u v$ is for the ultraviolet transitions.

$1 s-2 s$ ultraviolet transitions. The eventual uncertainty for the hyperfine structure is related to 5 parts in $10^{15}$ of the measured $1 s-2 s$ interval. A similar experiment for deuterium achieved an accuracy of the hyperfine interval related to even a smaller portion of the 'big' $1 s-2 s$ transition, namely 3 parts in $10^{15}$.

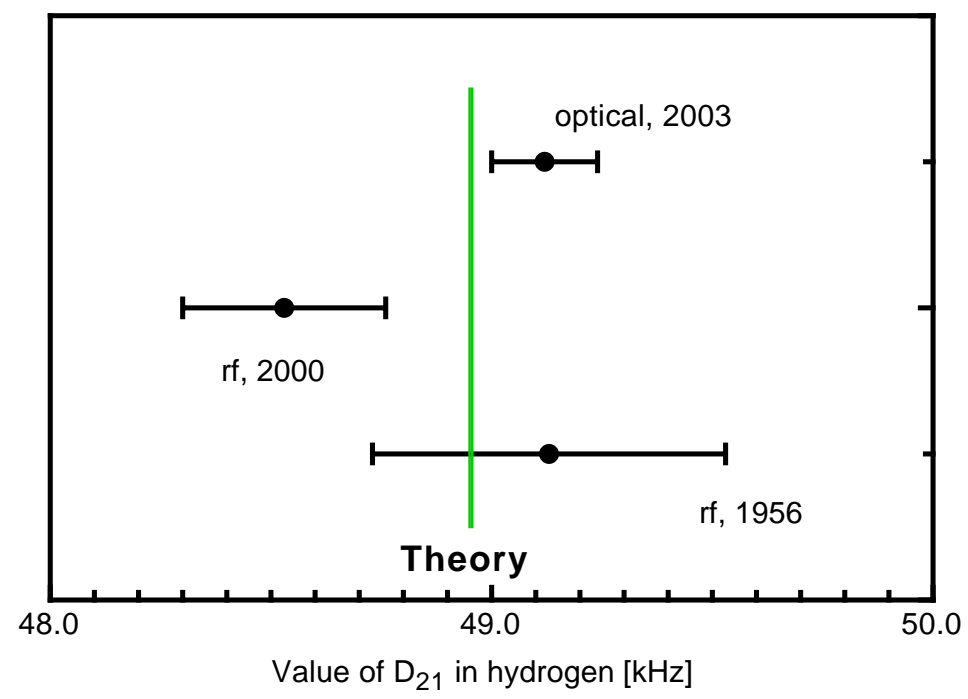

Fig. 19. Present status of experiment and theory of $D_{21}$ in the hydrogen atom. The results are labelled with the date of the measurement of the $2 s$ hyperfine structure. See Table 4 for references.

The comparison of theory to experiment for hydrogen, deuterium and helium3 ion is summarized in Figs. 19, 20 and 21. We acknowledge a substantial recent progress in experimental studies of neutral hydrogen and deuterium atoms, however, the helium ion data are still of the most interest since theory 


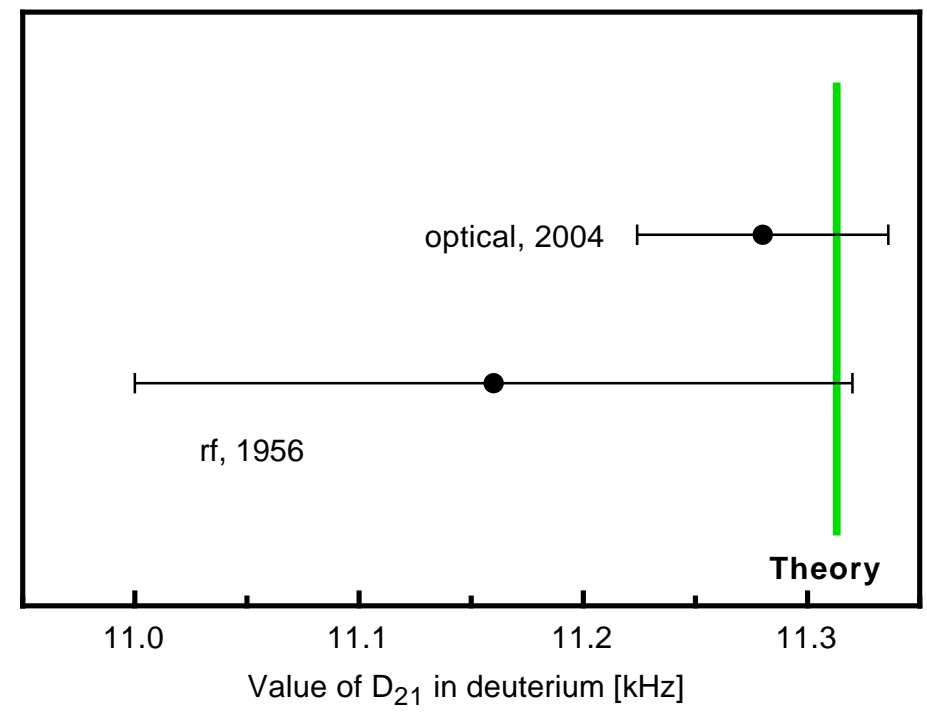

Fig. 20. Present status of experiment and theory of $D_{21}$ in the deuterium atom. The results are labelled with the date of the measurement of the $2 s$ hyperfine structure. See Table 4 for references.

and experiment are compatible.

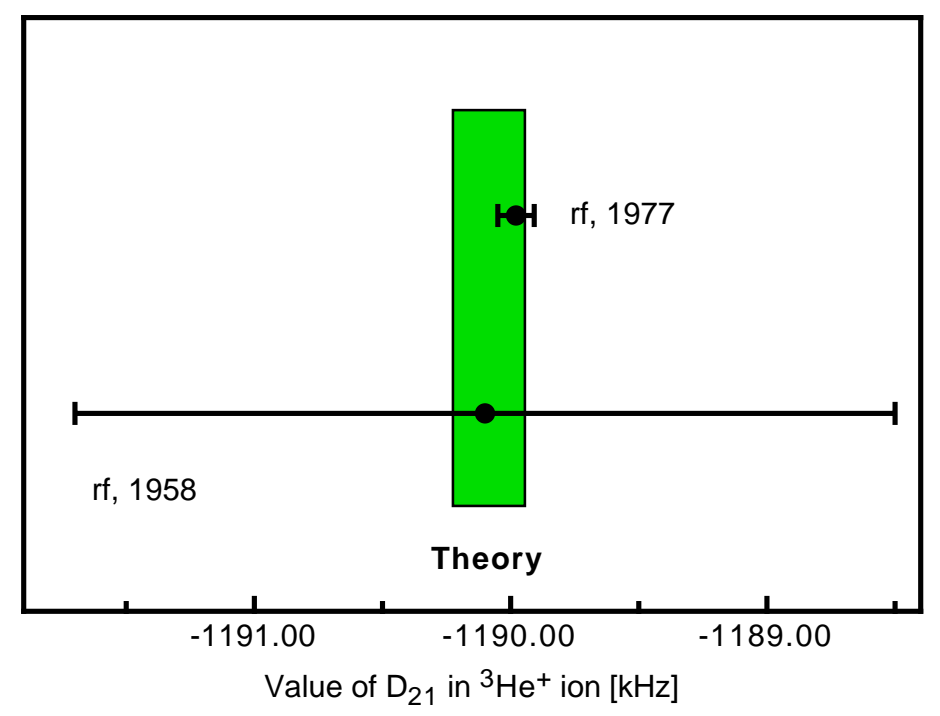

Fig. 21. Present status of experiment and theory of $D_{21}$ in the helium ion ${ }^{3} \mathrm{He}^{+}$. The results are labelled with the date of the measurement of the $2 s$ hyperfine structure. See Table 4 for references.

\section{Hyperfine splitting in the ground state of muonium}

Another possibility to eliminate the nuclear structure effects is based on studies of nucleon-free atoms. Such an atomic system can be formed of two leptons. 
Two atoms of the sort have been produced and studied for a while with high accuracy, namely, muonium and positronium. We consider the former below in this section, while the next section is devoted to the latter.

Muonium is a bound system of a positive muon and an electron. It can be produced with the help of accelerators. The muon lifetime is $2.2 \cdot 10^{-6} \mathrm{sec}$. However, it is sufficient to perform accurate spectroscopic measurements. The most accurately measured transition is the two-photon $1 s-2 s$ transition with an uncertainty of $4 \mathrm{ppb}$ [130]. The other accurately measured interval is the $1 s$ hyperfine splitting (12 ppb) [131]. A detailed review of muonium physics can be found in [132].

\begin{tabular}{lcc}
\hline Term & Fractional & $\Delta E$ \\
& contribution & {$[\mathrm{kHz}]$} \\
\hline$E_{F}$ & 1.000000000 & $4459031.88(50)(3)$ \\
$a_{e}$ & 0.001159652 & $5170.925(1)$ \\
QED2 & -0.000195815 & -873.145 \\
QED3 & -0.000005923 & -26.411 \\
QED4 & $-0.000000123(49)$ & $-0.548(218)$ \\
Hadronic & $0.000000054(1)$ & $0.241(4)$ \\
Weak & -0.000000015 & -0.067 \\
\hline Total & $1.000957830(49)$ & $4463302.88(51)(3)(22)$ \\
\hline
\end{tabular}

Table 9

Theory of the $1 s$ hyperfine splitting in muonium. The calculations [133] have been performed for $\alpha^{-1}=137.03599876(52)$ [134] and $\mu_{\mu} / \mu_{p}=3.18334517(36)$ which was obtained from the analysis of the data on the Breit-Rabi levels in muonium [131,135] (see Sect. 10.4) and muon precession [136]. The numerical results are presented for the frequency $E / h$.

Despite that the fractional accuracy of the measurement of the hyperfine interval is three times smaller than that of the $1 s-2 s$ transition, it is of a bigger interest. The uncertainty of the hyperfine measurement in muonium is indeed much smaller in absolute units $(53 \mathrm{~Hz}$ against $9.8 \mathrm{MHz}$ for the $1 s-2 s$ interval) and thus more sensitive to higher-order corrections. The Lamb shift of the $1 s$ state in muonium can be extracted from the optical measurement of the $1 s-2 s$ transition with fractional uncertainty of approximately $1 \%$, which is indeed not enough for any interesting QED test (it is larger, e.g., than the proton-finite-size contribution in the case of hydrogen). A real importance of the $1 s-2 s$ experiment is due to the determination of the muon mass which can be used in theoretical calculations for other QED tests such as the muonium hyperfine structure. We discuss the muon mass and magnetic moment in Sect. 10.4. 
Let us consider now the hyperfine interval of the ground state in muonium. The theoretical status is presented in Table 9. The budget of theoretical uncertainties in the case of muonium involves quite different sources and they are in part of experimental origin. The biggest source is in the calculation of the Fermi energy, accuracy of which is limited by the knowledge of the muon magnetic moment or the muon mass in proper units. To determine the muon magnetic moment $\left(g_{\mu} / 2\right)\left(e \hbar / 2 m_{\mu}\right)$ and its mass is essentially the same because the $g$ factor of a free muon is known well enough [137]. A smaller uncertainty in determination of the Fermi energy (see the number in the second brackets) is due to the fine structure constant. The theory up to the third order (i.e., $a_{e}, Q E D 2$ and QED3 terms) is well known (see references in Table 10). The nonrecoil part up to the third order is determined for the ground state by (26) and (28), while the recoil effects lead to an additional contribution

$$
\begin{aligned}
\Delta E_{1 s}(\mathrm{rec}) & =(Z \alpha) \frac{m}{M} \frac{E_{F}}{1+a_{\mu}} \times\left\{-\frac{3}{\pi} \ln \frac{M}{m}+(Z \alpha)\left[\ln \frac{1}{(Z \alpha)^{2}}-8 \ln 2+\frac{65}{18}\right]\right. \\
& \left.+\frac{\alpha}{\pi^{2}}\left[-2 \ln ^{2} \frac{M}{m}+\frac{13}{12} \ln \frac{M}{m}+\frac{21}{2} \zeta(3)+\frac{\pi^{2}}{6}+\frac{35}{9}\right]\right\}
\end{aligned}
$$

Table 10

\begin{tabular}{cclc}
\hline Correction & References & Correction & Reference(s) \\
\hline$\alpha(Z \alpha)^{2} E_{F}$ & {$[138,139,140]$} & $(Z \alpha)^{2}(m / M) E_{F}$ & {$[142]$} \\
$\alpha^{2}(Z \alpha) E_{F}$ & {$[141,66]$} & $\alpha(Z \alpha)(m / M) E_{F}$ & {$[143,144,145]$} \\
\hline
\end{tabular}

Recent references on the third order QED corrections for the ground state muonium hyperfine splitting. See [16] for detail.

The fourth-order corrections $(\triangle E(Q E D 4))$ have not yet been included either into a non-recoil term $E_{1 s}(Q E D)$ (see $(26)$ and (28)) or into a recoil term $\Delta E_{1 s}$ (recoil). They are the corrections that determine the uncertainty related to QED theory. These corrections are related to essentially the same diagrams as the $D_{21}(Q E D 4)$ contribution in the previous section. The results are summarized in Table 11. Unknown corrections are estimated according to [133]. Meantime some of them and in particular the corrections of relative order $\alpha^{3}(Z \alpha)$ [78] and $\alpha^{2}(Z \alpha)(m / M)$ [146] are under study and a few gauge invariant sets of diagrams have been already calculated. The muonium QED uncertainty is mainly due to the calculation of the recoil corrections of order $\alpha(Z \alpha)^{2} m / M$ and $(Z \alpha)^{3} m / M[148]$.

The muonium theory is not completely free of hadronic contributions, which appear because of intermediate hadronic states. That produces one more source of theoretical uncertainties. The effects of strong interactions in muonium are discussed in detail in Refs. $[133,161,162]$. They are relatively small, but their understanding is very important because of the intensive muon 


\begin{tabular}{ccc}
\hline Contribution & Numerical result & Reference \\
\hline$(Z \alpha)^{4}$ & $0.03 \mathrm{kHz}$ & {$[120]$} \\
$(Z \alpha)^{3} \frac{m}{M}$ & $-0.29(13) \mathrm{kHz}$ & {$[61,147,141,148,149,150]$} \\
$(Z \alpha)^{2}\left(\frac{m}{M}\right)^{2}$ & $-0.02(1) \mathrm{kHz}$ & {$[151,152,153]$} \\
$(Z \alpha)\left(\frac{m}{M}\right)^{3}$ & $-0.02 \mathrm{kHz}$ & {$[154]$} \\
$\alpha(Z \alpha)^{3}$ & $-0.52(3) \mathrm{kHz}$ & {$[155,125,156],[148],[157]$} \\
$\alpha(Z \alpha)^{2} \frac{m}{M}$ & $0.39(17) \mathrm{kHz}$ & {$[61,149,150]$} \\
$\alpha(Z \alpha)\left(\frac{m}{M}\right)^{2}$ & $-0.04 \mathrm{kHz}$ & {$[159,160]$} \\
$\alpha^{2}(Z \alpha)^{2}$ & $-0.04(2) \mathrm{kHz}$ & {$[133]$} \\
$\alpha^{2}(Z \alpha) \frac{m}{M}$ & $-0.04(3) \mathrm{kHz}$ & {$[161,162]$} \\
$\alpha^{3}(Z \alpha)$ & $\pm 0.01 \mathrm{kHz}$ & \\
$\tau$ lepton & $0.002 \mathrm{kHz}$ & \\
\hline$\Delta E(\mathrm{QED} 4)$ & $-0.55(22) \mathrm{kHz}$ & \\
\hline
\end{tabular}

Table 11

The fourth order corrections to the muonium hyperfine structure. The uncertainty is an rms sum of the partial uncertainties. The essential part of the contributions (see [133] for detail) have been found within the leading logarithmic approximation and their uncertainty is estimated for each contribution as a half-value of the leading term $[148,133]$. The $\tau$ lepton contribution is also included into the table and has an order $\alpha(Z \alpha) m M / m_{\tau}^{2}$.

sources expected in future [163] which can allow a dramatic increase of the accuracy of muonium experiments.

A comparison of theory and experiment for the muonium HFS interval is not an isolated problem, since it involves determination of the muon mass (magnetic moment) in proper units. We discuss problems of the determination of the muon mass and magnetic moment in Sect. 10.4 and a comparison of theory versus experiment for muonium hyperfine interval is presented in the Sect. 13 along with the other test of bound state QED for the hyperfine structure (see Table 19).

\section{Spectrum and annihilation of positronium and recoil effects}

Another pure leptonic atom is positronium. It can be produced at accelerators or using various radioactive positron sources. The lifetime of positronium depends on its state, on its orbital and spin $(S)$ quantum numbers. The lifetime for the $1 S$ state of parapositronium (this state with $S=0$ annihilates mainly into two photons) is $1.25 \cdot 10^{-10} \mathrm{sec}$, while orthopositronium (the $S=1$ state) 
in the $1 S$ state has a lifetime of $1.4 \cdot 10^{-7}$ sec because of the three-photon decays. A list of accurately measured positronium quantities contains the $1 S$ hyperfine splitting, the $1 S-2 S$ interval, the $2 S-2 P$ fine structure intervals, the lifetime of the $1 S$ state of para- and orthopositronium and several branching ratios of their decays. We note that since the nuclear spin effects are not suppressed, positronium has a structure of energy levels (in respect to their spin and angular momentum) rather similar to a two-electron system (such as the neutral helium atom) and thence we use capital letter for its orbital momentum.

Theoretical predictions for most of the values of interest and references to their comparison to experimental data are summarized in Table 12.

\begin{tabular}{lccc}
\hline Quantity & Leading term & Prediction & Figure \\
\hline$\Delta \nu(1 S-2 S)$ & $\frac{3}{8} \alpha^{2} m c^{2}$ & $1233607222.2(6) \mathrm{MHz}$ & 24 \\
$\Delta \nu_{H F S}(1 S)$ & $\frac{7}{12} \alpha^{4} m c^{2}$ & $203391.7(6) \mathrm{MHz}$ & 23 \\
\hline$\Delta \nu\left(2^{3} S_{1}-2^{3} P_{0}\right)$ & $\frac{5}{96} \alpha^{4} m c^{2}$ & $18498.25(9) \mathrm{MHz}$ & 28 \\
$\Delta \nu\left(2^{3} S_{1}-2^{3} P_{1}\right)$ & $\frac{7}{192} \alpha^{4} m c^{2}$ & $13012.41(9) \mathrm{MHz}$ & 28 \\
$\Delta \nu\left(2^{3} S_{1}-2^{3} P_{2}\right)$ & $\frac{23}{720} \alpha^{4} m c^{2}$ & $8625.70(9) \mathrm{MHz}$ & 28 \\
$\Delta \nu\left(2^{3} S_{1}-2^{1} P_{1}\right)$ & $\frac{1}{24} \alpha^{4} m c^{2}$ & $11185.37(9) \mathrm{MHz}$ & 28 \\
\hline$\Gamma(\mathrm{p}-\mathrm{Ps})$ & $\frac{1}{2} \alpha^{5} m c^{2}$ & $7989.62(4) \mu \mathrm{s}^{-1}$ & 26 \\
$\Gamma(\mathrm{o}-\mathrm{Ps})$ & $\frac{2\left(\pi^{2}-9\right)}{9 \pi} \alpha^{6} m c^{2}$ & $7.03996(2) \mu \mathrm{s}^{-1}$ & 25 \\
$\mathrm{Br}_{4 \gamma / 2 \gamma}(\mathrm{pPs})$ & $0.0278 \alpha^{2}$ & $1.439(2) \cdot 10^{-6}$ & 27 \\
\hline
\end{tabular}

Table 12

Theoretical predictions for positronium. Comparison to experimental data is presented in figures quoted in the table. The details and references for experiment can be found in $[166,167]$ while for theory in $[168,169]$ and below in Table 16 . The leading term for each value is presented in energy units.
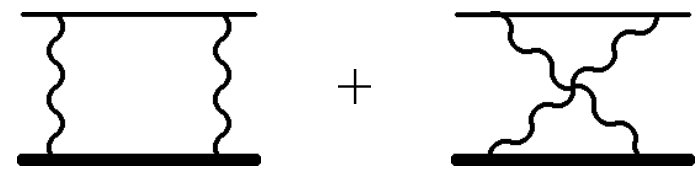

Fig. 22. Two-photon basic diagrams for the calculation of recoil contributions.

In the case of the atoms with a 'heavy' nucleus there is a significant difference between contributions to the Lamb shift and the hyperfine splitting. The hyperfine effects used to affect the Lamb shift and related intervals (such as the gross and fine structure) only slightly because the hyperfine splitting is of the order $(Z \alpha)^{4} m^{2} c^{2} / M$, while the Lamb shift is of the order $\alpha(Z \alpha)^{4} m c^{2} \ln (1 / Z \alpha)$. Since the effects due to the hyperfine shift are small, the higher order corrections to the HFS interval are not important for a study of the Lamb shift and related transitions. Entering into detail, we note that a crucial problem 
for theory of the hyperfine structure is a calculation of the recoil corrections, while for the Lamb shift they are not so important. The difference originates from the fact that if the momentum of integration is significantly below $M c$, the nuclear recoil corrections are rather of a kinematic origin similar (but much more complicated) to the reduced mass effects in the relativistic case (for the electron), since the nucleus can still be treated as a non-relativistic particle. If the momentum of integration is of the order of $M c$, the diagram for the basic block (see Fig. 22) has order $(Z \alpha)^{5}(m / M)^{2} m c^{2}$ and higher-order corrections to this block deliver some extra factors of $\alpha$ and $Z \alpha$. In other words, a recoil correction with a relativistic nucleus is of the second order of the electron-tonucleus mass ratio. That fact used to be 'hidden' in the standard notation. When one discusses the Lamb shift, the contributions used to be presented in the absolute units with all $(m / M)$ factors explicitly presented. Meantime, the relative units are commonly used for the corrections to the hyperfine structure and since the Fermi energy

$$
E_{F} \sim(Z \alpha)^{4} \frac{m}{M} m c^{2}
$$

includes a factor of $m / M$, a linear correction of relative order $m / M$ is actually the second order contribution in the absolute units.

Summarizing, we note that:

- studying the gross and fine structure and the Lamb shift in a conventional atom such as hydrogen, one needs to take into account the hyperfine effects with a relatively low accuracy;

- calculating the gross and fine structure and the Lamb shift, one does not need to learn much detail on theory of the second-order recoil corrections and to approach higher-order recoil effects;

- accuracy of theoretical predictions suffers from an uncertainty in the determination of the fundamental constants such as Rydberg constant $R_{\infty}$, the fine structure constant $\alpha$ and the nuclear mass and magnetic moment.

Positronium is an exceptional atom since $m / M=1$ and the situation strongly differs from the conventional atomic systems:

- studying the gross and fine structure of positronium, one needs to take into account the hyperfine effects with a high accuracy;

- the calculation of truly recoil corrections with relativistic nucleus is equally important for the gross and fine structure, as well as for the hyperfine structure and the lifetime calculations. Actually, the uncertainty of the calculations of the recoil effects determines the entire theoretical uncertainty for the most wanted spectroscopic characteristics in positronium.

The equality of $m / M$ to unity leads to another important feature of positro- 
nium tests for the bound state QED. Since the corrections of interest are enhanced $(m / M$ is not a suppressing factor any longer), the fractional accuracy for successful high-precision tests is now relatively low. As a result, in contrast to hydrogen, the interpretation of the measurements of the $1 S-2 S$ interval does not crucially involve knowledge of the Rydberg constant with high accuracy. A study of the hyperfine interval does not require a value of the fine structure constant $\alpha$ with high accuracy as it is in muonium. Since $m / M=1$, it is unnecessary to determine a mass ratio $m / M$ and a ratio of magnetic moments of the electron and nucleus, i.e., positronium ${ }^{8}$, in an additional experiment. In other words, positronium offers several high precision tests of bound state QED without any need to determine values of any fundamental or phenomenological constant with high accuracy.

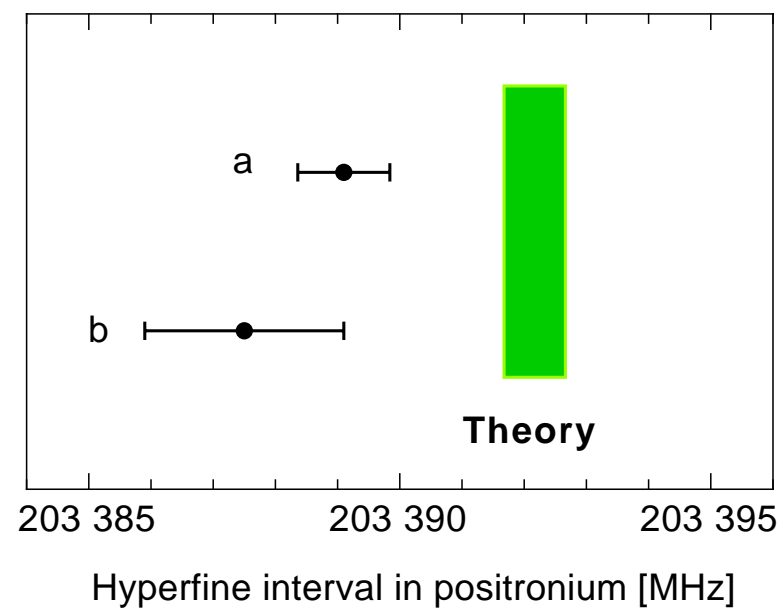

Fig. 23. The hyperfine splitting in the ground state of positronium. The Yale experiment $(a)$ was performed in 1984 [170] and the Brandeis one (b) in 1975 [171].

The HFS interval has been determined experimentally with the highest absolute accuracy among other positronium transitions. Present experimental data for the positronium hyperfine interval together with the theoretical result are depicted in Fig. 23. The theoretical status for the positronium hyperfine interval in the $1 S$ state is presented in detail in Table 13 . The dominant source of the uncertainty is the third-order corrections for positronium $(\triangle E(Q E D 3))$, which are known only in the logarithmic approximation. The diagrams under question are related to radiative-recoil and recoil corrections, and they are the same as those responsible for the uncertainty of muonium hyperfine interval $\left(\alpha(Z \alpha)^{2} m / M\right.$ and $\left.(Z \alpha)^{3} m / M\right)[61,168,114]$ (see the previous section for de-

$\overline{8}$ One may wonder what should happen if the CPT symmetry is broken and $m_{e} / m_{\bar{e}}$ and $\mu_{e} / \mu_{\bar{e}}$ are not exactly equal to unity. In principle, certain features of positronium might be sensitive to such a violation, however, special experiments with selective sensitivity to this effect would be needed. It is also clear, that such a CPT violation should show itself not just as a small mismatch in masses and magnetic moments, but as incorrectness of the basic equations (e.g., the Dirac equation). 
tail) . The fourth-order recoil terms of theory of heavy atoms become the third order corrections in positronium.

\begin{tabular}{lcccc}
\hline Term & & $\begin{array}{c}\text { Fractional } \\
\text { contribution }\end{array}$ & $\begin{array}{c}\Delta E \\
{[\mathrm{MHz}]}\end{array}$ & References \\
\hline$E_{F}$ & $\alpha^{4} m c^{2}$ & 1.0000000 & 204386.6 & \\
QED1 & $\alpha^{5} m c^{2}$ & -0.0049196 & -1005.5 & \\
QED2 & $\alpha^{6} m c^{2}$ & 0.0000577 & 11.8 & {$[184]$} \\
QED3 & $\alpha^{7} m c^{2}$ & $-0.0000061(22)$ & $-1.2(6)$ & {$[61,149,150,185]$} \\
\hline Total & & $0.9951321(22)$ & $203391.7(6)$ & \\
\hline
\end{tabular}

Table 13

Theory of the $1 S$ hyperfine interval in positronium. The numerical results are presented for the frequency $E / h$. The calculation of the second order terms was completed in Ref. [184], the leading logarithmic contributions were found in [61], while the next-to-leading logarithmic terms were achieved in $[149,150,185]$. The uncertainty is presented following $[168,169]$.

Studies of the spectrum and decay rates of positronium provide us with a number of strong tests of bound state QED, some of which are among the most accurate. Some theoretical predictions from Table 12 can be compared with accurate experimental data, a review of which can be found in Ref. [166]. The most accurately measured spectroscopic data are related to the ground state HFS (see Fig. 23) and to the $1 S-2 S$ interval (see Fig. 24 and Table 14). There are some minor discrepancies between experimental and theoretical data.

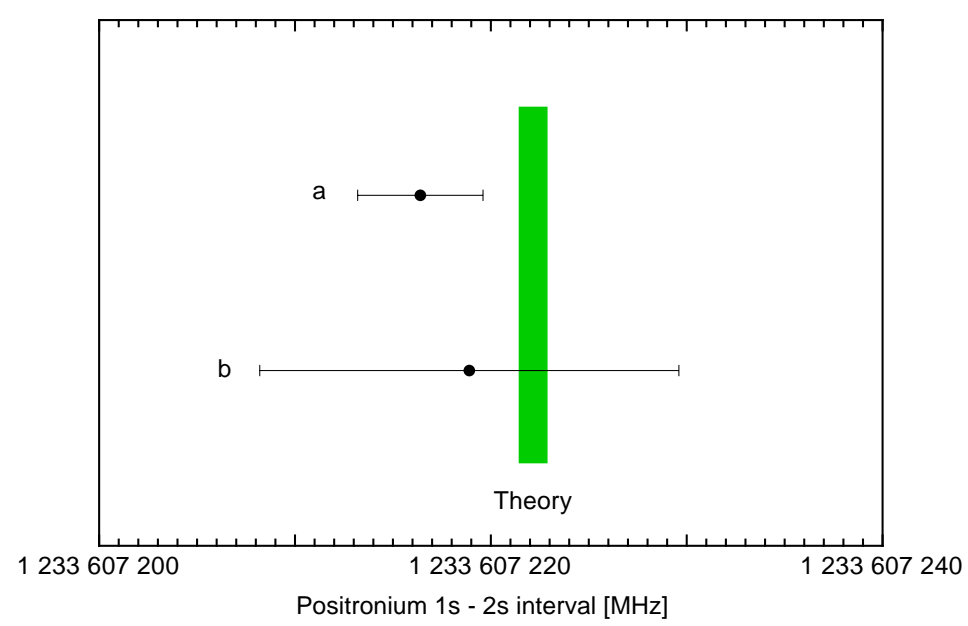

Fig. 24. Determination of the $1^{3} S_{1}-2^{3} S_{1}$ interval in positronium. The references here are: $a$ is for [172] and $b$ is for [173].

The experimental situation with the orthopositronium decay (see Fig. 25) had been not acceptable for a while but it has been recently improved [182]. The problem was a significant inconsistency of various experiments and long 


\begin{tabular}{cc}
\hline Term & $\Delta E$ \\
& {$[\mathrm{MHz}]$} \\
\hline$\alpha^{2} m c^{2}$ & 1233690735.1 \\
$\alpha^{4} m c^{2}$ & -82005.6 \\
$\alpha^{5} m c^{2}$ & -1501.4 \\
$\alpha^{6} m c^{2}$ & $-7.1,[174]$ \\
$\alpha^{7} m c^{2}$ & $1.2(6),[175,200]$ \\
\hline Total & $1233607222.2(6)$ \\
\hline
\end{tabular}

Table 14

Theory of the $1^{3} S_{1}-2^{3} S_{1}$ interval in positronium. The corrections $\Delta E$ are presented in the energy units, while their numerical values are given in the frequency units for $E / h$.

standing strong discrepancy between theory and the most accurate experimental data. The data presented in Fig. 25 include most recent vacuum results from Tokyo [183] and Ann Arbor [182] which are in a good agreement with theory (see Table 15). The original gas result from Ann Arbor (data point $c$ in Fig. 25) is corrected (data point $d$ ) according to the preliminary analysis in Ref. [166] but that is not a final result. Further examination is in progress and it seems that the final uncertainty will be bigger [179].

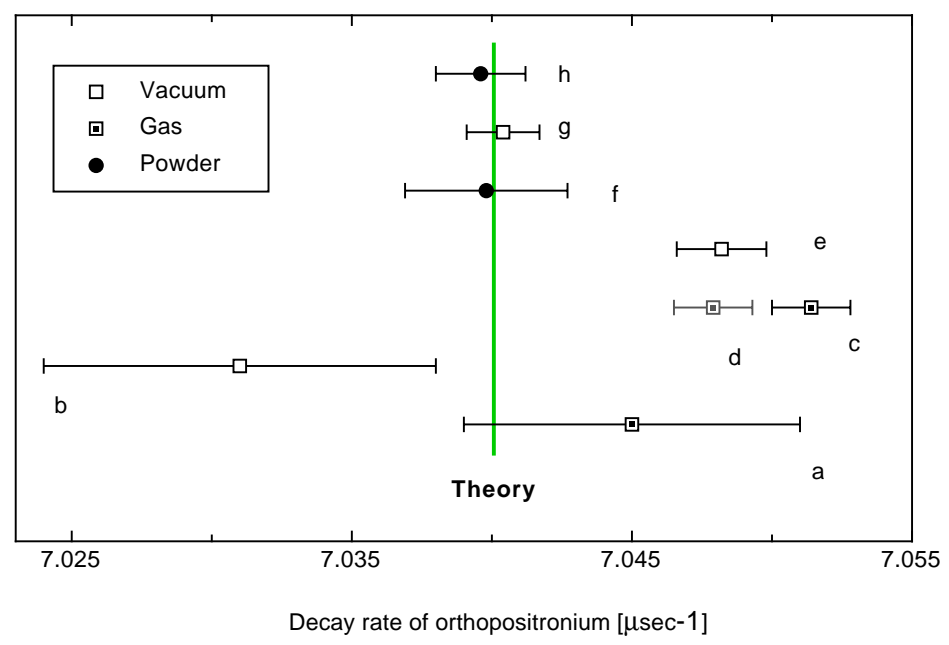

Fig. 25. Measurements of the annihilation decay rate of orthopositronium: $a-[176]$, $b-[177], c-[178], d-[166,179], e-[180], f-[181], g-[182], h-[183]$.

For the parapositronium decay theory and experiment are in perfect agreement (see Fig. 26 and Fig. 27). Most of positronium decay experiments were reviewed in Refs. [166,167].

These two papers also review experiments on the fine structure in positronium performed at $2^{3} S_{1}-2 P$ intervals which were less accurate than experiments at 


\begin{tabular}{llc}
\hline Contribution & $\begin{array}{c}\text { Decay rate } \\
\text { of orthopositronium } \\
{\left[\mu \mathrm{sec}^{-1}\right]}\end{array}$ & $\begin{array}{c}\text { Decay rate } \\
\text { of parapositronium } \\
{\left[\mu \mathrm{sec}^{-1}\right]}\end{array}$ \\
\hline$\Gamma^{(0)}$ & 7.21117 & 8032.50 \\
QED1 & -0.17230 & -47.25 \\
QED2 & $0.00111(1),[186]$ & $4.43(1),[187]$ \\
QED3 & $-0.00001(2),[61,188]$ & $-0.08(4),[61,188]$ \\
\hline Total & $7.03996(2)$ & $7989.62(4)$ \\
\hline
\end{tabular}

Table 15

Theory of the annihilation decay rate of ortho- and parapositronium (the $1 S$ state). The leading contributions are defined above in Table 12. The decay rate of ortho/parapositronium into five/four photons is included into corresponding QED2 terms.

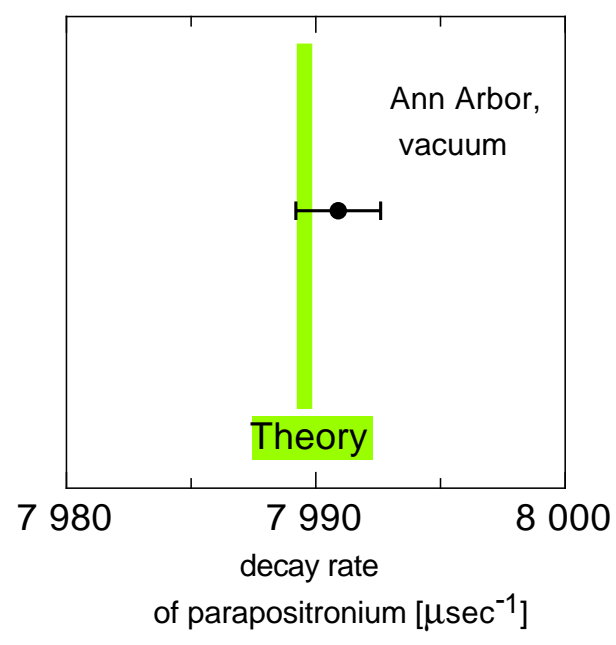

Fig. 26. Determination of the parapositronium decay rate. The experiment was performed in Ann Arbor in 1994 [189].

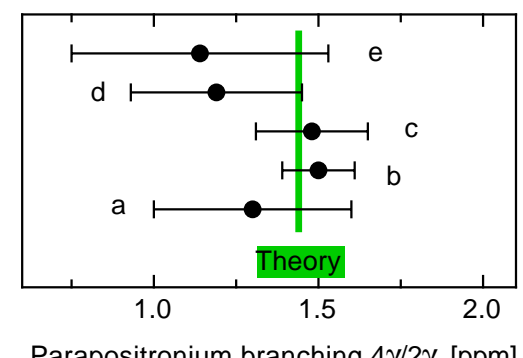

Fig. 27. Annihilation of parapositronium: branching $\operatorname{Br}(4 \gamma / 2 \gamma)$ measured around the world $(a-[190], b-[191], c-[192], d-[193], e-[194])$. 
$1 S$ HFS and $1 S-2 S$ intervals. Data on the fine structure are not as accurate as the results related to the $1 S$ hyperfine splitting and the $1 S-2 S$ interval but the progress is possible [166].

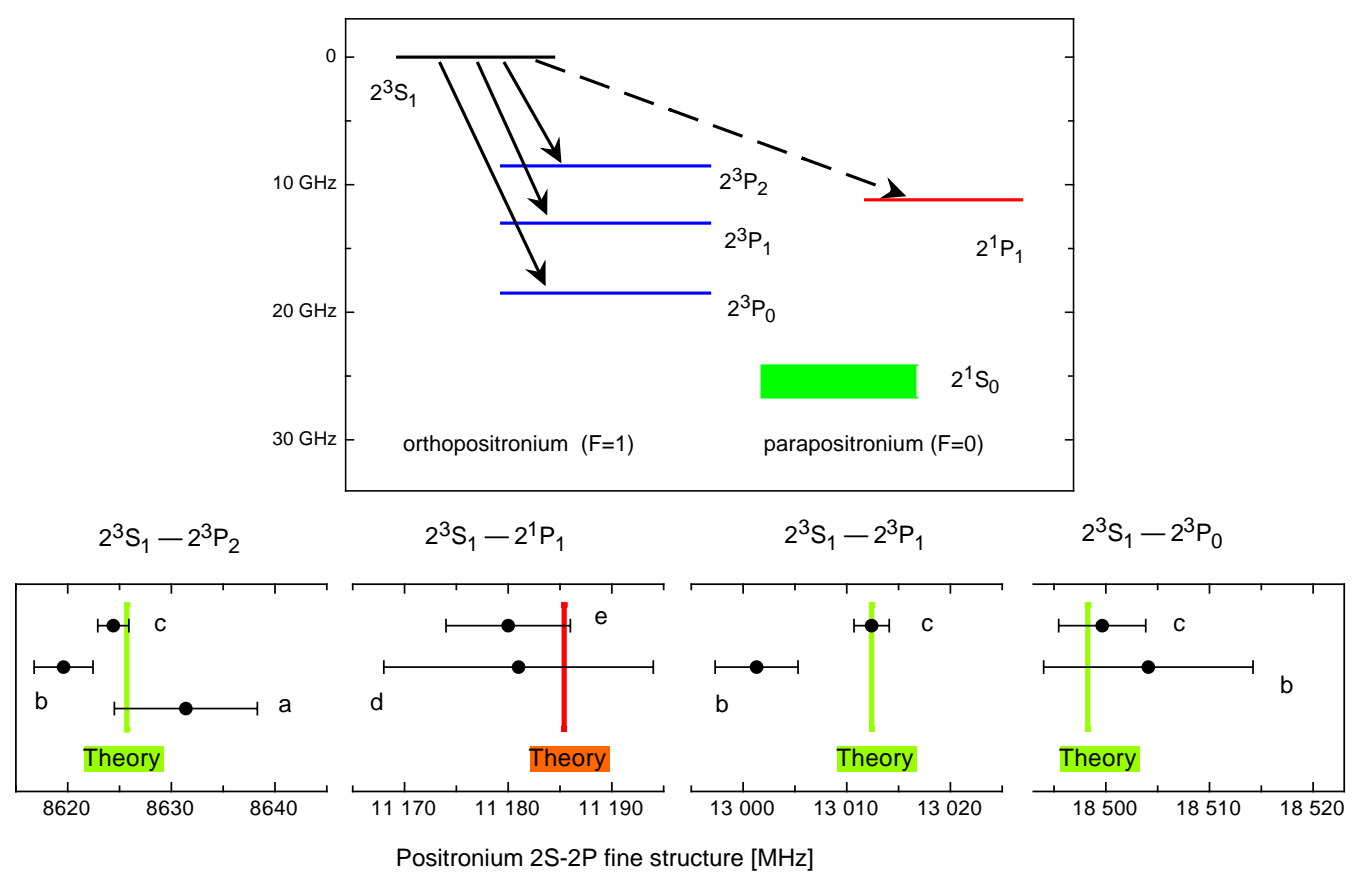

Fig. 28. The fine structure in positronium at $n=2$ : the energy levels and studied transitions. The $2^{3} S_{1}-2{ }^{1} P_{1}$ transition is forbidden for the free positronium atom and it was measured with an external magnetic field applied. The line width of the $2^{3} S_{1}$ state is of $1 \mathrm{MHz}$ and that of all $2 P$ states is of $50 \mathrm{MHz}$ and thus it cannot be seen in the scale of the top picture, while the width of parapositronium singlet $\left(2{ }^{1} S_{0}\right)$ being of $1.3 \mathrm{GHz}$ is clearly recognized. The references to the measurements are: $a-[195], b-[196], c-[197], d-[198]$ and $e-[199]$.

To conclude the section, let us discuss briefly the theory of the positronium decay rates and energy levels and in particular the uncertainty of their calculations. We collected all theoretical predictions in Table 12. The results were published and presented in different compilations. What we would like to emphasize here is our estimation of uncertainty.

The uncertainty of any perturbative theoretical calculation is determined by a plausible estimation of unknown higher-order corrections which are expected to have large coefficients. There is a number of corrections enhanced by a big double logarithmic factor $\ln ^{2} \alpha \simeq 24$ [61] and the higher-order terms should be studied to understand better the accuracy of theory. For the spectroscopy, the crucial order is $\alpha^{7} m c^{2}$, while in the case of the decay the theoretical problems are related to the corrections of the relative order $\alpha^{3}$.

For most of them not only the leading logarithmic corrections (e.g., in the case of the spectrum that is $\alpha^{7} m c^{2} \ln ^{2} \alpha$ ) are known, but also the next-to-leading 
Table 16

\begin{tabular}{cccc}
\hline Value & $\begin{array}{c}\text { Ref. to } \\
\alpha^{6} m c^{2}\end{array}$ & $\begin{array}{c}\text { Ref. to } \\
\alpha^{7} m c^{2} \ln ^{2} \alpha\end{array}$ & $\begin{array}{c}\text { Ref. to } \\
\alpha^{7} m c^{2} \ln \alpha\end{array}$ \\
& or $\alpha^{2} \cdot \Gamma^{0}$ & $\alpha^{2} \ln ^{2} \alpha \cdot \Gamma^{0}$ & $\alpha^{2} \ln \alpha \cdot \Gamma^{0}$ \\
\hline $1 S-2 S$ & {$[174]$} & {$[175,200]$} & unknown \\
fine structure & {$[174]$} & {$[175,200]$} & unknown \\
$1 S$ HFS & {$[184]$} & {$[61]$} & {$[149,150,185]$} \\
\hline$\Gamma(\mathrm{p}-\mathrm{Ps})$ & {$[187]$} & {$[61]$} & {$[188]$} \\
$\Gamma(\mathrm{o}-\mathrm{Ps})$ & {$[186]$} & {$[61]$} & {$[188]$} \\
\hline
\end{tabular}

References to recent progress in the positronium theory. The contributions to the spectrum are classified by the absolute energy units (such as $\alpha^{6} m c^{2}$ ), while those to the decay are presented in units of the leading contribution $\Gamma^{0}$ (see Table 12).

term $\left(\alpha^{7} m c^{2} \ln \alpha\right)$. We should emphasis, however, that such a knowledge cannot reduce the uncertainty. The leading term originates (the most probably, but not always) from a single source and its magnitude is a natural characteristic of the correction, while the next-to-leading term used to be a result of a summation of or a cancellation between different contributions and can be sometimes small. But that smallness is misleading and the constant following the single logarithm is not necessary small. Our estimation of the uncertainty $[168,169]$ is based on a value of the double logarithmic term in any case.

\section{The $g$ factor of an electron and a nucleus in a light hydrogen-like atom}

\subsection{General consideration}

Not only a spectrum of simple atoms can be studied with a high accuracy. Among other quantities accessible to high precision measurements are the magnetic moments of bound subatomic particles. The only way to determine a value of a magnetic moment precisely (i.e., with uncertainty at the level of $0.01 \mathrm{ppm}$ or below) is to measure a certain frequency proportional to the magnetic moment and applied magnetic field. Since the magnetic field can be neither measured nor calculated with uncertainty essentially below $0.1 \mathrm{ppm}$, it is possible to measure accurately only certain ratios of the magnetic moments. Another option is to compare a spin precession frequency related to the magnetic moment with the ion cyclotron frequency. Two-body atomic systems provide us with an opportunity to study the magnetic moments of their bound composites and in certain experiments to compare their magnetic moments to 
each other.

The interaction of an atom with a weak homogenous magnetic field $\mathbf{B}$ can be expressed in terms of an effective Hamiltonian. For an $s$ state in the two-body atom such a Hamiltonian for spin variables has the form (see, e.g., [9])

$$
H_{\mathrm{magn}}=g_{e}^{\prime} \frac{e \hbar}{2 m}(\mathbf{s} \cdot \mathbf{B})-g_{N}^{\prime} \frac{Z e \hbar}{2 M}(\mathbf{I} \cdot \mathbf{B})+J_{\mathrm{HFS}}(\mathbf{s} \cdot \mathbf{I})
$$

where $\mathbf{s}$ stands for the spin of an electron, $\mathbf{I}$ is for the nuclear spin, $g_{e(N)}^{\prime}$ is for the $g$ factor of a bound electron (nucleus) in the two-body atom and $J_{\mathrm{HFS}}$ is the hyperfine constant ${ }^{9}$. We remind here, that $m$ and $M$ are the masses of the electron and the nucleus, respectively.

We present the $g$ factor of a bound electron in the form $[60,202,203]$

$$
g^{\prime}=2 \cdot(1+a+b)
$$

where $a$ is the anomalous magnetic moment of a free electron, while $b$ stands for the binding correction. As it has been known for a while $[9,204]$, even in absence of QED effects the $g$ factor of a bound electron is not equal to the free Dirac value (namely, $g_{\text {Dir }}^{(0)}=2$ ). The leading radiative and recoil effects were later examined in $[205,206]$.

Three basic situations with the two-body atomic systems at presence of a homogeneous magnetic field are of practical interest:

- a 'conventional' low- $Z$ atom with a non-zero nuclear spin (hydrogen, deuterium, tritium, helium-3 ion);

- muonium, a pure leptonic unstable atom with a relatively light nucleus $\left(m_{e} / m_{\mu} \simeq 1 / 207 \gg m_{e} / m_{p} \simeq 1 / 1836\right)$

- a medium- $Z$ hydrogen-like ion with a spinless nucleus (the ions of carbon-12 and oxygen-16) with a possible expansion of experimental activity down to helium-4 and beryllium-10 ions and up to calcium-40 ion.

The bound $g$ factors in light atoms are now known up to the fourth-order

$\overline{9}$ The notation for the $g$ factors is different in particle and nuclear physics. For ions one needs to apply a different notation in which the magnetic Hamiltonian reads

$$
H_{\mathrm{magn}}=g_{e}^{\prime} \frac{e \hbar}{2 m}(\mathbf{s} \cdot \mathbf{B})-g_{N}^{\prime} \frac{e \hbar}{2 m_{p}}(\mathbf{I} \cdot \mathbf{B})+J_{\mathrm{HFS}}(\mathbf{s} \cdot \mathbf{I})
$$

where $g_{N}^{\prime}$ differs indeed from that in Eq. (38). To avoid confusion, in this paper $g$ is used only for the case of particles (electrons, muons, protons etc.), while if we need the $g$ factor for a nucleus, we introduce additional notation (see e.g., (cf. 34)). 


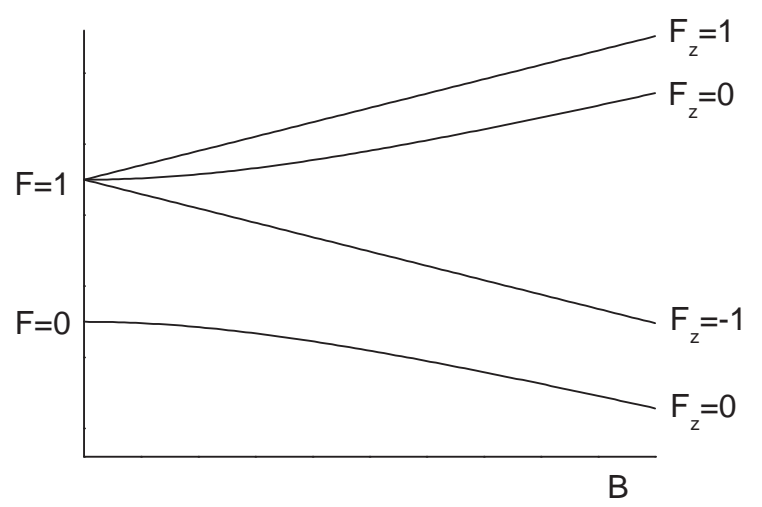

Fig. 29. Sublevels $E_{\text {magn }}\left(F, F_{z}\right)$ of the hyperfine structure in the $n s$ state of hydrogen or another hydrogen-like atom with nuclear spin $1 / 2$ at a homogenous magnetic field $B$. The related equation was first derived by Breit and Rabi (see [9]).

corrections [207] including the terms of the order $\alpha^{4}, \alpha^{3} m / M$ and $\alpha^{2}(m / M)^{2}$ and thus the relative uncertainty is substantially better than a part in $10^{8}$. All these corrections are of kinematic origin and there is no need for a higher accuracy and thus for any higher order corrections for a moment.

For the medium- $Z$ ions some corrections of higher orders are important for the comparison with experimental data and for applications to the determination of the fundamental constants. These corrections involve essential bound state QED effects. The 'kinematic origin' reads that they are a result of a 'simple' quantum mechanical problem of two point-like particles bound by a Coulomb interaction with their actual values of the masses and magnetic moments. 'Free QED' corrections are a part of kinematic effects since we consider the actual values of the magnetic moments which include the anomalous magnetic moment. 'Bound state QED' corrections are related to somewhat beyond this approximation - to non-Coulomb additions to the interactions (as, e.g., the Uehling potential), structure effects (as, e.g., the electron self-energy). Such higher order corrections were studied and in particular the linear correction in $\alpha$ has been known exactly in all orders of $Z \alpha$ as well as some higher order recoil contributions.

10.2 Hydrogen and its isotopes: the isotopic effects for the $g$ factor of a bound electron

Few experiments were performed with hydrogen and its isotopes, as well as with the helium-3 ion. Dependence of energy sublevels on the magnetic field is presented in Fig. 29 for the $n s$ state in a two-body atom with nuclear spin 1/2 (hydrogen, tritium, helium-3 ion, muonium). 


\begin{tabular}{clcl}
\hline Value & Experiment & Reference & Theory \\
\hline$g(\mathrm{H}) / g_{0}(e)$ & $1-17.709(13) \cdot 10^{-6}$ & {$[208,209]$} & $1-17.694 \cdot 10^{-6}$ \\
$g(\mathrm{D}) / g(\mathrm{H})$ & $1-7.22(3) \cdot 10^{-6}$ & {$[210]$} & $1-7.24 \cdot 10^{-6}$ \\
$g(\mathrm{~T}) / g(\mathrm{H})$ & $1-10.7(15) \cdot 10^{-6}$ & {$[211]$} & $1-9.7 \cdot 10^{-6}$ \\
$g\left({ }^{4} \mathrm{He}^{+}\right) / g_{0}(e)$ & $1-70.88(30) \cdot 10^{-6}$ & {$[208,209,212,213,214,215]$} & $1-70.91 \cdot 10^{-6}$ \\
\hline
\end{tabular}

Table 17

The bound electron $g$ factor in light hydrogen-like atoms: a comparison of theory and experiment. Here, $g_{0}(e)$ stands for the magnetic moment of a free electron and it contains the anomalous magnetic moment $a=\alpha / 2 \pi+\ldots$

The hyperfine sublevels were successfully studied by a few different ways.

- For example, one is a study of a few splittings related to the same atom.

- Other experiments on the hydrogen-like atoms dealt with the $g$ factors of an electron bound in different atoms studying a kind of isotopic dependence in the electron $g$ factor.

- One more approach was realized for muonium: the field-induced energy intervals were measured at the magnetic field calibrated against the proton spin precession (see Sect. 10.4).

The isotopic experiments were performed on hydrogen, deuterium and tritium measured at the same applied magnetic field. The results are summarized in Table 17. The helium-ion result there involved a chain of several intermediate comparisons including experiments with neutral rubidium. All corrections to the electron $g$ factor up to the fourth order are known, with an exception. There are two contradicting results for the $(Z \alpha)^{2}(m / M)^{2}$ term which can be potentially nuclear-spin-dependent. One group [216] performed a semi-classical evaluation and reported on a nuclear-spin-independent result, while the others [217] claimed a difference between the results for the nuclear spin $I=1 / 2$ and $I=1$. For the nuclear spin $1 / 2$ both reproduced the well known result [206]. Hopefully, for deuterium their discrepancy is somewhat smaller than the experimental uncertainty, while the other two-body atoms of interest involve the nuclear spin $I=1 / 2$ for which the result in this order is well established.

Setting the nuclear spin equal to $1 / 2$, the equations for the bound $g$ factor in two-body atom read $[207,218]$

$$
\begin{aligned}
g_{e}^{\prime} & =g_{e} \cdot\left\{1-\frac{(Z \alpha)^{2}}{3}\left[1-\frac{3}{2} \frac{m}{M}\right]+\frac{\alpha(Z \alpha)^{2}}{4 \pi}-\frac{(Z \alpha)^{2}(1+Z)}{2}\left(\frac{m}{M}\right)^{2}\right. \\
& \left.-\frac{5 \alpha(Z \alpha)^{2}}{12 \pi} \frac{m}{M}-(0.289 \ldots) \times \frac{\alpha^{2}(Z \alpha)^{2}}{\pi^{2}}-\frac{(Z \alpha)^{4}}{12}\right\}
\end{aligned}
$$

and 


$$
\begin{aligned}
g_{N}^{\prime} & =g_{N} \cdot\left\{1-\frac{\alpha(Z \alpha)}{3}\left[1-\frac{m}{2 M} \frac{3+4 a_{N}}{1+a_{N}}\right]\right. \\
& \left.+\alpha(Z \alpha)\left(\frac{m}{M}\right)^{2}\left(-\frac{1}{2}-\frac{Z}{6} \frac{3-4 a_{N}}{1+a_{N}}\right)-\frac{97}{108} \alpha(Z \alpha)^{3}\right\},
\end{aligned}
$$

where the anomalous magnetic moment of the nucleus $a_{N}$ is defined as

$$
\begin{aligned}
\mu_{\mathrm{Nucl}} & =2\left(1+a_{N}\right) \frac{Z e \hbar}{2 m} I \\
& \simeq 2\left(1+a_{N}\right) \frac{Z}{A} \mu_{N} I
\end{aligned}
$$

where, we remind, $M$ is the nuclear mass, $I$ is its spin, while $\mu_{N}$ in the nuclear magneton. Thus $a_{p} \simeq 1.793 \ldots, a_{d} \simeq-0.143 \ldots, a_{t} \simeq 7.916 \ldots$ and $a_{h} \simeq$ $-1.184 \ldots$ (the latter value is for a helion, the nucleus of the ${ }^{3} \mathrm{He}$ ). We note that appearance of the factor of $\left(1+a_{N}\right)$ in the denominator is rather artificial: a more natural (from the theoretical point of view) expression is of the form

$$
g_{N}^{\prime}=2 \times\left(1 \cdot g_{1}+a_{N} \cdot g_{a}\right)
$$

however, due to applications is it more convenient to present the result as above (i.e., as a multiplicative correction to $g_{N}$, a free value of the $g$ factor).

We remark also that some of the listed values of the nuclear anomalous magnetic moments are of large numerical values (as, e.g., $a_{t}$ ). That is not an exception. The triton magnetic moment is essentially a proton magnetic moment (the total spin of two neutrons is zero) somehow perturbed. However, the magneton related to the triton is approximately a third part of the proton's since the charge of the proton and triton is the same, but the triton mass is threefold higher.

10.3 Hydrogen and deuterium: determination of the proton and deuteron magnetic moments

Studies of a few HFS intervals in the same atom in the presence of the magnetic field were performed for hydrogen [219] and deuterium [220] and their results 
were found to be ${ }^{10}$

$$
\frac{\mu_{e}(\mathrm{H})}{\mu_{p}(\mathrm{H})}=\frac{g_{e}(\mathrm{H})}{g_{p}(\mathrm{H})} \frac{\mu_{B}}{\mu_{N}}=658.2107058(66)
$$

and

$$
\frac{\mu_{e}(\mathrm{D})}{\mu_{d}(\mathrm{D})}=2143.923565(23)
$$

The bound state QED contributions are small and the quoted experiments were not designed to test them. The purpose was different, namely, to determine the proton magnetic moment which is widely used as a probe magnetic moment for other QED tests (such as the muonium hyperfine structure $[131,135]$, the anomalous magnetic moment of a muon [137]) or for other measurements (such as of the deuteron magnetic moment [129] etc.). In fact, the hydrogen experiment [219] with the theoretical evaluation performed in [218] has delivered the most accurate value of a free ratio of the electron-to-proton magnetic moments

$$
\frac{\mu_{p}}{\mu_{e}}=658.2106859(66)
$$

and the proton $g$ factor. The result of the evaluation in [218] is somewhat shifted from the CODATA value [21] due to recently obtained higher-order corrections $[221,222,207]$ and improvement in determination of the electronto-proton mass ratio discussed below (see Sect. 11).

In the case of deuteron there are competitive NMR experiments on HD spectroscopy. The experimental results published about twenty years ago in [223,224,225,226] were obtained in NMR high-pressure experiments and they are not free of possible systematic effects. We start a program of additional experiments [129] and we hope to study those systematic effects in more detail and achieve reliable data. The present situation is summarized in Fig. 30.

\footnotetext{
${ }^{10}$ Here and further in this section we ignore the direction of the spin and the magnetic moment and thus the sign of some $g$ factors and ratios of magnetic moments. To simplify notations, we also drop the prime sign when the bound system is explicitly specified as, e.g., in the case of $g(\mathrm{H})$.
} 


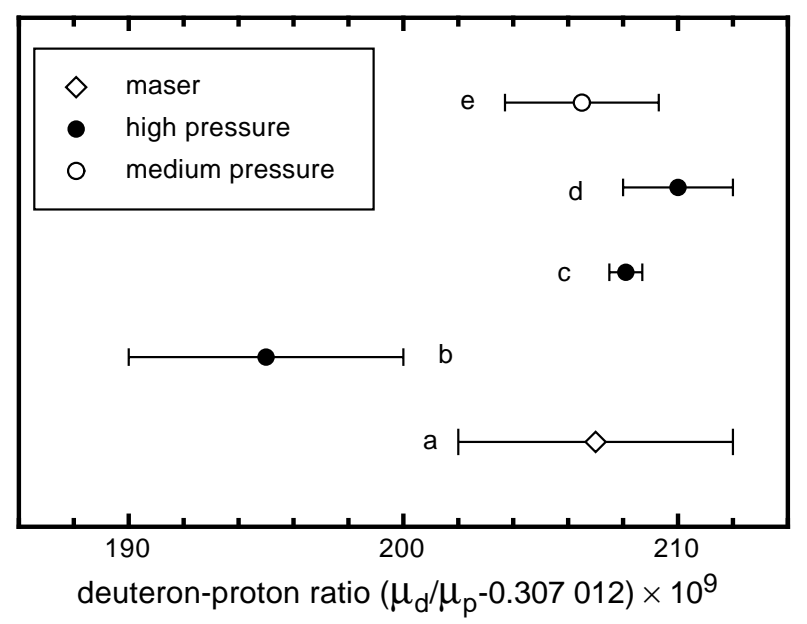

Fig. 30. The deuteron-to-proton ratio of the magnetic moments: maser result $a$ is taken from $[219,220,21]$, the other results are from NMR spectroscopy of hydrogen deuteride HD. Result $b$ is related to an experiment with an admixture of helium-3 at pressure of 60-80 atm [225], while results $c$ and $d$ correspond to measurements on pure HD gas at pressure of $130 \mathrm{~atm}$ with/without rotation of the sample [226]. The high-pressure results here have an uncertainty corrected for an inaccuracy in the calculation of screening effects (see [129] for detail). The result $e$ is a recent result obtained at a medium pressure $(10 \mathrm{~atm})$ in order to check effects of the pressure dependence [129]. The systematic effects due to the pressure shift are under study and have not been included into the uncertainty budget.

10.4 The $g$ factors of an electron and a muon in muonium and the determination of the muon mass

A difference between theory for muonium and for 'conventional' atoms is due to a higher importance of recoil effects (since the nucleus, namely a muon, is approximately tenfold lighter than a proton) and a possibility to expand the muon magnetic moment in $\alpha$. After expanding $a_{\mu}$, the result for the bound muon $g$ factor reads $[207,114]$

$$
\begin{aligned}
g_{e}(\mathrm{Mu}) & =g_{e}^{(0)} \cdot\left\{1-\frac{(Z \alpha)^{2}}{3}\left[1-\frac{3}{2} \frac{m}{M}\right]+\frac{\alpha(Z \alpha)^{2}}{4 \pi}-\frac{(Z \alpha)^{2}(1+Z)}{2}\left(\frac{m}{M}\right)^{2}\right. \\
& \left.-\frac{5 \alpha(Z \alpha)^{2}}{12 \pi} \frac{m}{M}-\frac{(Z \alpha)^{4}}{12}-(0.289 \ldots) \cdot \frac{\alpha^{2}(Z \alpha)^{2}}{\pi^{2}}\right\}
\end{aligned}
$$

and

$$
g_{\mu}(\mathrm{Mu})=g_{\mu}^{(0)} \cdot\left\{1-\frac{\alpha(Z \alpha)}{3}\left[1-\frac{3}{2} \frac{m}{M}\right]\right.
$$




$$
\left.-\frac{\alpha(Z \alpha)(1+Z)}{2}\left(\frac{m}{M}\right)^{2}+\frac{\alpha^{2}(Z \alpha)}{12 \pi} \frac{m}{M}-\frac{97}{108} \alpha(Z \alpha)^{3}\right\}
$$

where the $g$ factor of a free lepton includes the anomalous magnetic moment $g_{e, \mu}^{(0)}=2 \cdot\left(1+a_{e, \mu}\right)$.

Studies of the Breit-Rabi levels (see Fig. 29) in muonium delivered the best datum on the electron-to-muon mass ratio needed for a determination of the fine structure constant $\alpha$ from the muonium hyperfine structure (see Sect. 8). Equation (38) has been applied $[131,135]$ to determine the muon magnetic moment and the muon mass by measuring the splitting of sublevels in the hyperfine structure of the $1 s$ state in muonium in a homogenous magnetic field. Since the magnetic field was calibrated via the spin precession of a proton, the muon magnetic moment was measured in units of the proton magnetic moment, and the muon-to-electron mass ratio was derived as

$$
\frac{m_{\mu}}{m_{e}}=\frac{\mu_{\mu}}{\mu_{p}} \frac{\mu_{p}}{\mu_{B}} \frac{1}{1+a_{\mu}}
$$

The results on the muon mass extracted from the Breit-Rabi formula are among the most accurate (see Fig. 31). Still there is a more precise value available derived from the muonium hyperfine structure after a comparison of the experimental result with theoretical calculations. However, the latter is of reduced interest, since the most important application of the precise value of the muon-to-election mass is to use it as an input for calculations of the muonium hyperfine structure while testing QED or determining the fine structure constants $\alpha$. The adjusted CODATA result [21] in Fig. 31 was extracted from the muonium hyperfine structure studies and in addition used a somewhat over-optimistic estimation of the theoretical uncertainty (see for detail [133]).

\section{The $g$ factor of a bound electron in a hydrogen-like ion with a spinless nucleus and a determination of $m_{e} / m_{p}$}

A study of the $g$ factor of a bound electron in a medium- $Z$ hydrogen-like ion with a spinless nucleus offers a comparison of the electron spin precession frequency affected by QED effects and the ion cyclotron frequency. That can be used to test QED theory as well as to determine the electron-to-proton

mass ratio. An important feature of such a study is that in contrast to most of the other tests it is possible to do both without any interference between these two tasks. That is possible because of an opportunity to perform experiments with different hydrogen-like ions. 


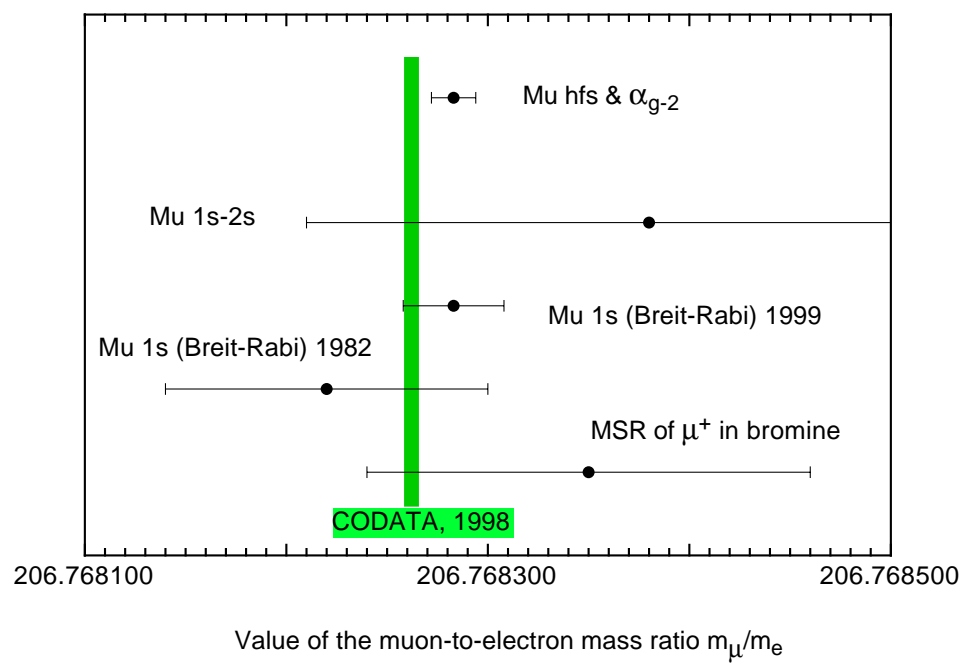

Fig. 31. The muon-to-electron mass ratio. The most accurate result obtained from comparison of the measured hyperfine interval in muonium [131] to a theoretical calculation [133] performed with $\alpha_{g-2}^{-1}=137.03599876(52)$ [134]. The results derived from study of the Breit-Rabi sublevels are related to two experiments performed at LAMPF in 1982 [135] and 1999 [131]. The others are taken from the measurement of the $1 s-2 s$ interval in muonium [130], muon-spin-precession-resonance (MSR) study of a muon in bromine [136] and from the CODATA adjustment [21].

Since the anomalous magnetic moment of a free electron is known with high accuracy $[227,21]$

$$
a_{e}=0.001159652187(4)
$$

to calculate the bound $g$ factor defined in Eq. (39), one has to obtain a bound correction $b$. A summary of the calculation of the bound corrections is presented in Table 18. We mainly follow here our consideration in [207] ${ }^{11}$. In particular, the uncertainty of unknown two-loop contributions is taken from [230], where the theoretical uncertainty was reasonably estimated for the first time. The results of the one-loop self-energy are achieved in different ways for different atoms. For lighter elements (helium, beryllium) it is based on fitting [207] data of [231], while for heavier ions we use the results of [232]. The other results are taken from [230] (for the one-loop vacuum polarization; see also [233]), [203] (for the nuclear correction and the electric part of the light-bylight scattering (Wichmann-Kroll) contribution; see also [233]), [234] (for the magnetic part of the light-by-light scattering contribution) and [235] (for the recoil effects).

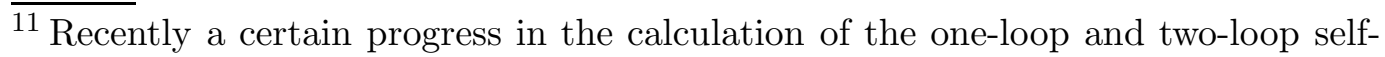
energy contribution [228] as well as of the the magnetic part of the light-by-light scattering contribution [229] was achieved. Since presently the experimental uncertainty dominates, this improvement of theory has no consequences for a moment. 
Table 18

\begin{tabular}{cc}
\hline Ion & $g$ \\
\hline${ }^{4} \mathrm{He}^{+}$ & $2.0021774067(1)$ \\
${ }^{10} \mathrm{Be}^{3+}$ & $2.0017515747(2)$ \\
${ }^{12} \mathrm{C}^{5+}$ & $2.0010415900(4)$ \\
${ }^{16} \mathrm{O}^{7+}$ & $2.0000470203(8)$ \\
${ }^{18} \mathrm{O}^{7+}$ & $2.0000470211(8)$ \\
\hline
\end{tabular}

The bound electron $g$ factor in low- $Z$ hydrogen-like ions with spinless nucleus.

Before comparing the theory to experimental data, let us shortly describe certain details of the experiment crucial for its interpretation. To determine a quantity like the $g$ factor, one needs to measure a certain field-induced frequency (such as the spin precession frequency) at some known magnetic field $B$. It is clear that there is no way to determine the strength of the magnetic field with a high accuracy directly. The conventional way is to measure two frequencies and to compare them. The frequencies measured in the GSI-Mainz experiment [236] are the ion cyclotron frequency

$$
\omega_{c}=\frac{(Z-1) e}{M_{i}} B
$$

where $M_{i}$ is the ion mass, and the Larmor spin precession frequency for a hydrogen-like ion with a spinless nucleus

$$
\omega_{L}=g_{b} \frac{e}{2 m_{e}} B
$$

where $g_{b}$ is the bound electron $g$ factor.

Combining them, one can obtain a result for the $g$ factor of a bound electron

$$
\frac{g_{b}}{2}=(Z-1) \frac{m_{e}}{M_{i}} \frac{\omega_{L}}{\omega_{c}}
$$

or for the electron-to-ion mass ratio

$$
\frac{m_{e}}{M_{i}}=\frac{1}{Z-1} \frac{g_{b}}{2} \frac{\omega_{c}}{\omega_{L}} .
$$

To measure the frequencies, one has to apply a certain magnetic filed and, in principle, it should be as homogenous as possible. However, the measurement of a frequency consists of two important parts: an induction of a certain magnetic transition by an accurately calibrated perturbation and a detection of 
the transition. The homogeneity of the field, which is a crucial requirement of the induction, is in a contradiction with the detection scheme, which requires a non-homogeneity of the magnetic field. In particular, a certain $z$-dependence of the field was applied providing ion oscillations in a vertical direction which were detected. Since the oscillation frequency depends on the magnetic state that allows to identify the state and thus to detect the transition. The breakthrough in a study of the bound $g$ factor [236] was a result of a space separation of the transition and detection areas, which gives an opportunity to support a very homogenous field for the transitions and afterwards to measure the response function in a separate area with $z$ gradients.

Today the most accurate value of $m_{e} / M_{i}$ (without using data from the experiments on the bound $g$ factor) is based on a measurement of $m_{e} / m_{p}$ realized in a Penning trap [237] with a fractional uncertainty of $2 \mathrm{ppm}$. The accuracy of measurements of $\omega_{c}$ and $\omega_{L}$ as well as of the calculation of $g_{b}$ (as shown in [202]) is substantially better. That means that it is preferable to apply Eq. (53) to determine the electron-to-ion mass ratio [238]. Applying the theoretical value for the $g$ factor of the bound electron and using the experimental results for $\omega_{c}$ and $\omega_{L}$ in hydrogen-like carbon [236] and some auxiliary data related to the proton and ion masses from [21], we arrive at the following values

$$
\frac{m_{p}}{m_{e}}=1836.1526731(10)
$$

and

$$
m_{e}=0.00054857990929(31) \mathrm{u},
$$

which slightly differ from those in [238]. The present status of the determination of the electron-to-proton mass ratio is summarized in Fig. 32.

In [202] it was also suggested in addition to the determination of the electron mass to check theory by comparing the $g$ factor for two different ions. In such a case the uncertainty related to $m_{e} / M_{i}$ in Eq. (52) vanishes. The theoretical uncertainty is chiefly related to uncalculated higher-order terms. Since various higher-order corrections are proportional to $Z^{4}$ or so, even a small change in $Z$, such as, e.g., a comparison of the carbon result $(Z=6)$ to the oxygen one $(Z=8)$ is a quite sensitive probe for the value of the higher-order terms.

Thus, combining the experimental results for carbon [236] and oxygen [239] ions

$$
g\left({ }^{12} \mathrm{C}^{5+}\right) / g\left({ }^{16} \mathrm{O}^{7+}\right)=1.0004972731(15),
$$

one can verify the reliability of the estimation of the theoretical accuracy. The 


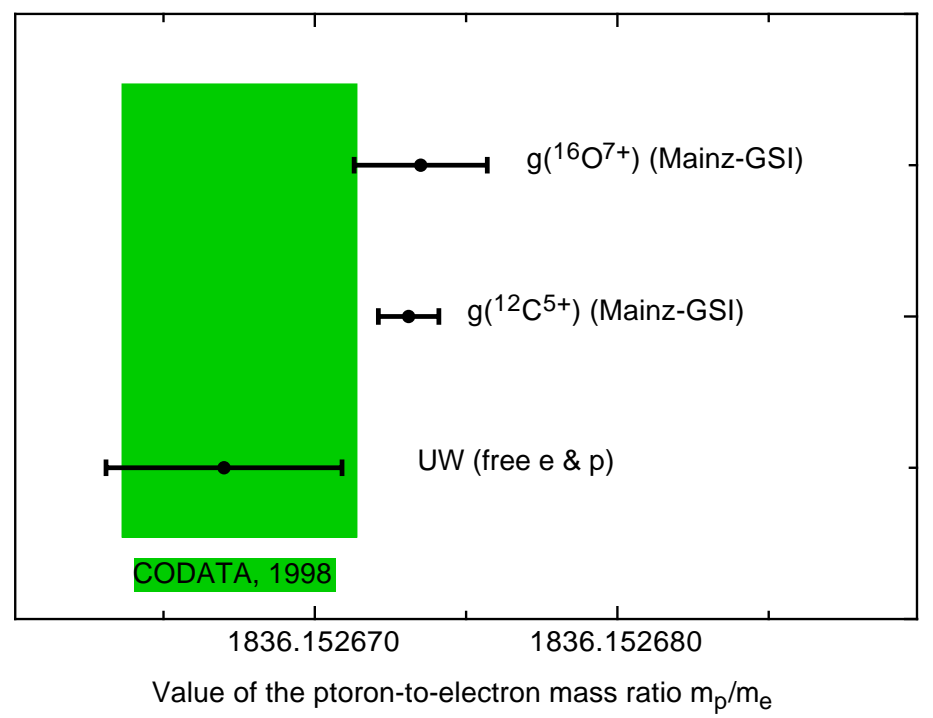

Fig. 32. The proton-to-electron mass ratio. The theory of the bound $g$ factor is taken from Table 18, while the experimental data on the $g$ factor in carbon and oxygen are from $[236,239]$. The Penning trap result from University of Washington is from [237].

experiment appears to be in perfect agreement with theory

$$
g\left({ }^{12} \mathrm{C}^{5+}\right) / g\left({ }^{16} \mathrm{O}^{7+}\right)=1.0004972733(9) .
$$

Unfortunately, the authors of the experiments did not present this value directly [239]. They presented results for the $g$ factors of both ions separately. In both cases the experimental uncertainties contain systematic and statistical contributions. The dominant contribution is due to the electron mass and it should vanish in the ratio. It is also not good to determine $m_{e} / M_{i}$ from one experiment (let us say from carbon's) and use as an input for the other (oxygen's). In such a way additionally to the experimental uncertainties the experimental $g$ factor for the oxygen would contain a theoretical uncertainty for the carbon $g$ factor.

That is why it is so important to speak in terms of the ratio of the $g$ factors. The experimental ratio is to be determined by experimentalists who perfectly know the correlations between the systematic sources of two measurements, while the theoretical ratio is to be determined by theorists and that allows to take into account correlations in the estimations of unknown higher-order terms.

The estimation in Eq. (56) is based on our understanding of the papers $[236,239]$ and on a private communication [240]. The agreement between theory and experiments means that we have a reasonable estimate of the uncalculated higher-order terms. Note, however, that for metrological applications 
it is preferable to study lower $Z$ ions (hydrogen-like helium-4 and beryllium10) to eliminate these higher-order terms if the experimental accuracy will increase.

As mentioned above, the leading radiative corrections are of kinematic origin and in particular the contributions of order $\alpha(Z \alpha)^{2}$ and $\alpha^{2}(Z \alpha)^{2}$ are related to a relativistic bound-state Coulomb problem for an electron and muon with non-zero anomalous magnetic moments, which are determined by free QED. The leading bound-state QED effects are of order $\alpha(Z \alpha)^{4}$ (the one-loop contributions) and $\alpha^{2}(Z \alpha)^{4}$ (the two-loop contributions). The former are known with sufficient accuracy, while lack of accurate results for the latter determines the uncertainty of the theory. That is compatible with a test of the Lamb shift theory at the level of a percent (for the hydrogen-like ions of carbon and oxygen). That is indeed not accurate enough for any interesting bound-state QED test. And in fact that is a big advantage of the experiments on the bound electron $g$ factor for determination of $m_{e} / m_{p}$. In a typical situation (the Lamb shift and the Rydberg constant; the muonium hyperfine structure and the fine structure constant; the helium fine structure and $\alpha$ ) we have to try to verify QED calculations and to determine a certain fundamental constant within the same experiment. In the case of the determination of the electron-to-proton mass ratio the QED theory is relatively simple. We still need to prove QED calculations, but we have not yet approached the crucial problem of the Lamb shift theory, namely, a problem of higher-order two-loop corrections. However, with sufficient theoretical progress this problem can be studied with present experimental accuracy if the experiment will be turned to higher $Z$, in particular, to $Z=20-30$. The higher-order bound-state QED effects should contribute at a detectable level and may be successfully studied. Comparing these higher $Z$ results with $Z=6,8$ and lower $Z$ one can cancel an uncertainty due to the determination of the electron-to-proton mass ratio. Thus, the experimental verification of bound-state QED theory and the determination of $m_{e} / m_{p}$ can be clearly separated.

\section{A determination of the fine structure constant $\alpha$ by means of QED and atomic physics}

Precision measurements accompanied by accurate theoretical calculations strongly interfere with high precision determinations of certain fundamental constants. Those are needed in order to interpret the theoretical expressions in terms of measurable quantities. The fine structure constant $\alpha$ plays a basic role in QED tests. In atomic and particle physics there are several ways to determine its value. The results are summarized in Fig. 33. One method based on the muonium hyperfine interval was briefly discussed in Sect. 8. A value of the fine structure constant can also be extracted from the neutral-helium fine 
structure [241,242,243] and from a comparison of theory [134] and experiment [227] for the anomalous magnetic moment of an electron $\left(\alpha_{g-2}\right)$. The latter value has been the most accurate one for a while and there was a long search for another competitive value. Since the value of $\alpha_{g-2}$ is based on one-group experiment [227] and one-group theory [244] there has been a long standing concern on reliability of this value and a search for its confirmation, in particular, via an independent competitive determination of the fine structure constant. At present, the second best value $\left(\alpha_{\mathrm{Cs}}\right)$ in the list of the most precise results for the fine structure constant comes from recoil spectroscopy [245]. It is only three times less accurate than $\alpha_{g-2}$. We note, however, that the result has not yet been published in a refereed journal, but only in several conference proceedings [245]. Since results in proceedings are less reliable than those published in the journals, we look forward to the eventual publication.

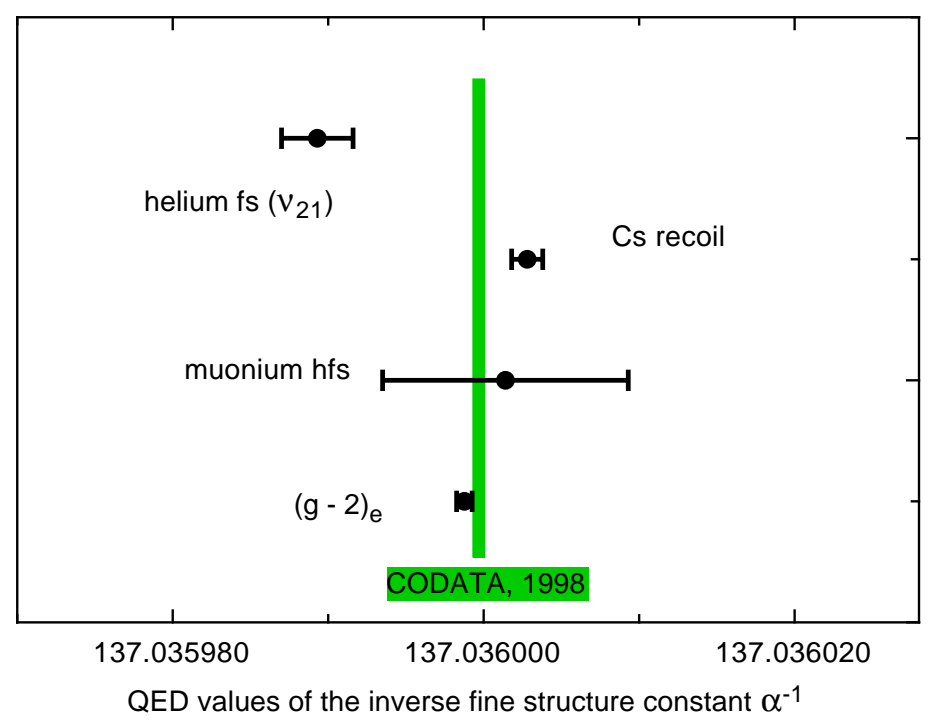

Fig. 33. The value of the fine structure constant $\alpha$ as determined by means of atomic physics and QED.

We would like to consider briefly the use and the significance of the recoil result for the determination of the fine structure constant. Absorbing and reemitting a photon, an atom, if it is initially in rest, gains some kinetic energy which can be determined through a measurement of the shift of the emitted frequency in respect to the absorbed one. The recoil shift depends on relative directions of two photons

$$
\Delta f=-C(\theta) \frac{h f^{2}}{2 M_{a} c^{2}},
$$

where $M_{a}$ is the mass of the atom, and under conditions of the experiment $[245] C=2$.

That is a measurement of the frequency $\Delta f$ with high accuracy that was the 
aim of the photon recoil experiment [245]. Combining the absorbed frequency and the shift $\Delta f$, it is possible to determine a value of atomic mass in frequency units, i.e., a value of $M_{a} c^{2} / h$. In experiment [245] the atom was cesium and the transition was the D1 line. The atomic mass of cesium is known very well in atomic units (or in units of the proton mass) [246] and the D1 line was accurately measured in [247]. A value of $M_{a} c^{2} / h$ may be compared to the Rydberg constant $R_{\infty}=\alpha^{2} m_{e} c / 2 h$ and if the electron mass in proper units is known, that eventually delivers us a value of $\alpha$. A determination of the electron mass is more complicated than of any atomic mass because of a gap of three orders of magnitude between masses of an electron and a proton. At present, the biggest uncertainty of the recoil photon value of $\alpha_{\mathrm{Cs}}$ comes from the experiment [245], however, accuracy in the determination of the electron mass is still significant since that is the second important source of the uncertainty.

The success of the $\alpha_{\mathrm{Cs}}$ determination was sometimes ascribed to the fact that $\alpha_{g-2}$ is a QED value, derived with the help of QED theory of the anomalous magnetic moment of an electron, while the photon recoil result is free of QED. We would like to emphasize that the situation is not so simple and the importance of the involvement of QED is rather exaggerated. What is more important is that the uncertainty of $\alpha_{g-2}$ originates from understanding of the electron behavior in the Penning trap and it dominates over any QED uncertainty. For this reason, the value of $\alpha_{\mathrm{Cs}}$, based on $m_{p} / m_{e}$ from another Penning trap experiment [237] and actually obtained by the same group, which measured the value of the anomalous magnetic moment of electron [227], can actually be correlated with $\alpha_{g-2}$. The result

$$
\alpha_{\mathrm{Cs}}^{-1}=137.03600028(10)
$$

presented in Fig. 33 is obtained using $m_{p} / m_{e}$ from Eq. (54). The value of the proton-to-electron mass ratio found this way is free of the problems with an electron in the Penning trap, but a certain QED theory is involved. However, one has to realize that the QED uncertainty for the $g$ factor of a bound electron and that for the anomalous magnetic moment of a free electron are completely different. The bound state QED theory deals with relatively simple Feynman diagrams but in Coulomb field and in particular to improve theory of the bound $g$ factor, we need better understanding of Coulomb effects for 'simple' two-loop QED diagrams. In contrast, for the free electron no Coulomb field is involved, but the uncertainty of the computation arises because of the accuracy in a numerical calculation of a big number of complicated four-loop diagrams. There is no correlation between these two kinds of calculations. The words 'simple' and 'complicated' are related to the diagrams, but not to the simplicity and difficulties of the calculations. Both kinds of calculations are quite difficult but in a different way. The crucial free diagrams are complicated 
diagrams built of simple blocks. The key bound QED contributions correspond to a simple diagram constructed of complicated blocks.

The two best values, $\alpha_{g-2}$ and $\alpha_{\mathrm{Cs}}$, agree with the muonium result but contradict to the helium value (see Fig. 33). The latter is presented in Fig. 33 rather to show a potential accuracy of the application of the helium fine structure method. The helium fine structure of the $1 s 2 p$ states involves few transitions and in particular so-called 'small interval' $\left(\nu_{01}\right)$ and 'big interval' $\left(\nu_{12}\right)$. A comparison of theory against experiment is presented in Fig. 34. One can match a certain scatter of the experimental data and a bad agreement of theory and experiment. In principle, one could speculate on a special value of the fine structure constant which will set an agreement of theory and experiment. However, the contradiction for $\nu_{01}$ and $\nu_{12}$ is at the same level in absolute units (from 5 to $20 \mathrm{kHz}$ ) and differs in fractional units since the 'big interval' is more than tenfold bigger than the 'small interval'. The contradiction for the 'small interval' is too big to be explained by any acceptable shift in the value of $\alpha$. The situation is quite unclear but, still, the recent improvement of theoretical accuracy remains quite promising [241,242].

There is a number of other values of the fine structure constant (see $[21,24]$ for detail). Some of them (based on a measurement of the gyromagnetic ratio of a proton and a helion and on a determination of $h / m_{n}$ ) involve some QED theory and experiments, however, the uncertainty of all of them is completely determined by metrological problems due to electric standards and a production of a perfect silicon crystal for the Avogadro project. The other electrical determinations, based on a measurement of the quantum Hall resistance in the SI units, needs no QED. Since the accuracy of those results is substantially below that of $\alpha_{g-2}$ and since their uncertainty is related to the electric, magnetic and material standards we do not consider them here. An updated review of QED and non-QED determination of the fine structure constant can be found in [23].

\section{Higher-order logarithmic corrections and the uncertainty of the bound-state QED calculations}

\subsection{Hyperfine structure in light hydrogen-like-atoms}

To summarize QED tests related to the hyperfine structure, we present in Table 19 the data related to the HFS interval in the $1 s$ state of positronium and muonium and to the specific difference $D_{21}$ in hydrogen, deuterium and helium-3 ion. As we noted in Sects. 7, 8 and 9, their theoretical uncertainty

is related to recoil effects of relative order $\alpha^{3}(\mathrm{~m} / M)$. In the case of helium 

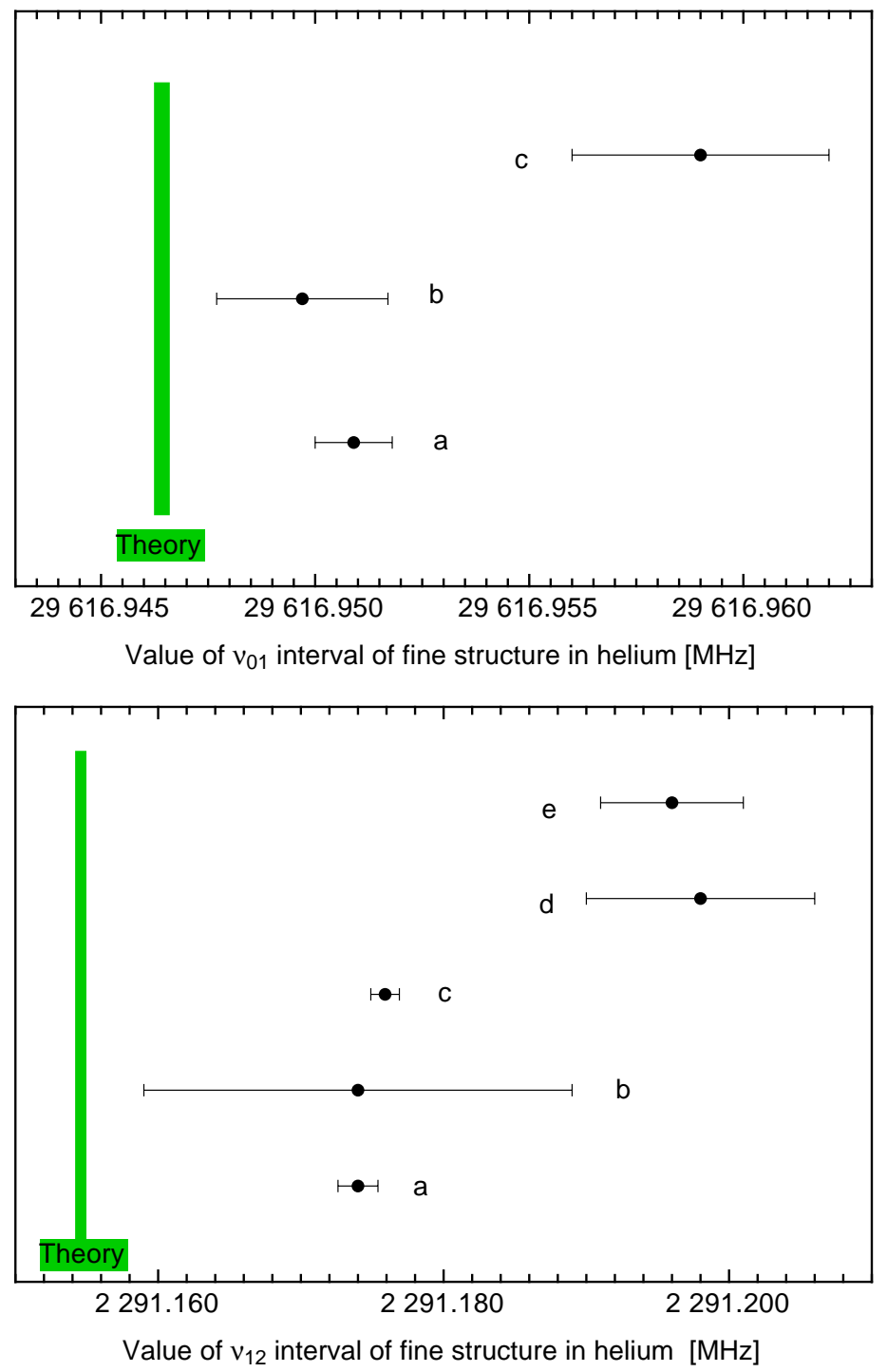

Fig. 34. The fine structure in neutral helium. $\nu_{01}$ stands for $1 s 2 p^{3} P_{0}-{ }^{3} P_{1}$ and $\nu_{12}$ for $1 s 2 p^{3} P_{1}-{ }^{3} P_{2}$. The theory is presented according to [241,242], the experimental data are taken $a$ from [243], $b$ from [248], $c$ from [249], $d$ from [250] and $e$ from [251]. Less accurate data are not included.

$D_{21}$ the higher-order one-loop and two-loop effects are also important, as well as higher-order nuclear corrections. The theoretical accuracy supersedes the experimental one (except of the case of the $D_{21}$ HFS interval in the helium3 ion, for which the theoretical uncertainty was recently increased after its reconsideration $[115,116])$. The theory agrees with experiment very well. A certain expection is the positronium case where the agreement is not perfect. 


\begin{tabular}{lcccc}
\hline Atom & $\begin{array}{c}\text { Experiment } \\
{[\mathrm{kHz}]}\end{array}$ & $\begin{array}{c}\text { Theory } \\
{[\mathrm{kHz}]}\end{array}$ & $\Delta / \sigma$ & $\begin{array}{c}\sigma / E_{F} \\
{[\mathrm{ppm}]}\end{array}$ \\
\hline Hydrogen, $D_{21}$ & $49.13(13),[106]$ & $48.953(5)$ & 1.3 & 0.09 \\
Hydrogen, $D_{21}$ & $48.53(23),[107]$ & & -1.8 & 0.16 \\
Hydrogen, $D_{21}$ & $49.13(40),[108]$ & & 0.4 & 0.28 \\
Deuterium, $D_{21}$ & $11.280(56),[109]$ & $11.3125(10)$ & -0.6 & 0.17 \\
Deuterium, $D_{21}$ & $11.16(16),[110]$ & & -1.0 & 0.49 \\
${ }^{3} \mathrm{He}^{+}$ion, $D_{21}$ & $-1189.979(71),[111]$ & $-1190.08(15)$ & 0.6 & 0.02 \\
${ }^{3} \mathrm{He}^{+}, D_{21}$ & $-1190.1(16),[112]$ & & -0.01 & 0.19 \\
\hline Muonium, $1 s$ & $4463302.78(5)$ & $4463302.88(55)$ & -0.18 & 0.11 \\
Positronium, $1 s$ & $203389100(740)$ & $203391700(500)$ & -2.9 & 4.4 \\
Positronium, $1 s$ & $203397500(1600)$ & & -2.5 & 8.2 \\
\hline
\end{tabular}

Table 19

A comparison of experiment and theory of the hyperfine structure in light hydrogenlike atoms. The numerical results are presented for the frequency $E / h$. In the $D_{21}$ case the reference is given only for the $2 s$ hyperfine interval, since for all the atoms of interest the $1 s$ HFS interval was measured more accurately (see table 4 for more detail).

\subsection{Crucial higher-order corrections}

The precision physics of light simple atoms provides us with an opportunity to check higher-order effects of perturbation theory. The highest-order terms important for the comparison of theory and experiment are collected in Table 20. The results for the energy levels and decay rates of interest. There are also a few dimensionless quantities of interest, which are mainly the $g$ factors. The uncertainty of the $g$ factor of the bound electron in carbon and oxygen is related to $\alpha^{2}(Z \alpha)^{5} m c^{2}$ corrections in the energy units, while for calcium the crucial order is $\alpha^{2}(Z \alpha)^{6} m c^{2}$ if the experiment will reach the same level of accuracy.

While some of the crucial corrections presented in Table 20 are completely known, others are not. Many of the listed corrections and in particular $\alpha(Z \alpha)^{6} \mathrm{~m}^{2} / M^{3}$ and $(Z \alpha)^{7} m^{2} / M^{3}$ for the hyperfine structure in muonium and helium ion, $\alpha^{2}(Z \alpha)^{6} m$ for the Lamb shift in hydrogen and helium ion, $\alpha^{7} m$ for positronium have been known in a so-called logarithmic approximation. In other words, only the terms with the highest power of a 'large' logarithm (e.g., $\ln (1 / Z \alpha) \sim \ln (M / m) \sim 5$ in muonium) have been taken into account. We note that the 'large' corrections should have an essentially non-relativistic origin. Truly relativistic corrections used to have a few factors $Z \alpha / \pi$ instead of 


\begin{tabular}{|c|c|}
\hline Value & $\begin{array}{l}\text { Order } \\
{\left[m c^{2}\right]}\end{array}$ \\
\hline Hydrogen, deuterium (gross structure) & $\alpha(Z \alpha)^{7}, \alpha^{2}(Z \alpha)^{6}$ \\
\hline Hydrogen, deuterium (fine structure) & $\alpha(Z \alpha)^{7}, \alpha^{2}(Z \alpha)^{6}$ \\
\hline Hydrogen, deuterium (Lamb shift) & $\alpha(Z \alpha)^{7}, \alpha^{2}(Z \alpha)^{6}$ \\
\hline${ }^{3} \mathrm{He}^{+}$ion $(2 s$ HFS $)$ & $\begin{array}{c}\alpha(Z \alpha)^{7} m / M, \alpha(Z \alpha)^{6} m^{2} / M^{2} \\
\alpha^{2}(Z \alpha)^{6} m / M,(Z \alpha)^{7} m^{2} / M^{2}\end{array}$ \\
\hline${ }^{4} \mathrm{He}^{+}$ion (Lamb shift) & $\alpha(Z \alpha)^{7}, \alpha^{2}(Z \alpha)^{6}$ \\
\hline Muonium (1s HFS) & $\begin{array}{c}(Z \alpha)^{7} m^{2} / M^{2}, \alpha(Z \alpha)^{6} m^{2} / M^{2} \\
\alpha(Z \alpha)^{7} m / M\end{array}$ \\
\hline Positronium (1s HFS) & $\alpha^{7}$ \\
\hline Positronium (gross structure) & $\alpha^{7}$ \\
\hline Positronium (fine structure) & $\alpha^{7}$ \\
\hline Para-positronium (decay rate) & $\alpha^{7}$ \\
\hline Ortho-positronium (decay rate) & $\alpha^{8}$ \\
\hline Para-positronium ( $4 \gamma$ branching) & $\alpha^{8}$ \\
\hline Ortho-positronium ( $5 \gamma$ branching) & $\alpha^{8}$ \\
\hline
\end{tabular}

Table 20

A comparison of QED theory and experiment: the crucial orders of magnitude for the energy levels and decay rates in units of $m c^{2}$ (see [60] for more detail).

$Z \alpha$, which is a characteristic non-relativistic factor for the Coulomb problem, and thus they are numerically suppressed. First calculations of logarithmically enhanced higher-order terms were performed for higher-order corrections in muonium HFS, hydrogen Lamb shift, positronium energy level and decay rates in our paper [61] and were successfully developed by various authors in $[148,34,252,175,200,124,113]$. By now even some non-leading logarithmic terms have been evaluated by several groups $[149,150,185,67,68]$.

Such a value of the parameter as $\sim 1 / 5$ is sufficient to make a good first approximation. We used to estimate accuracy of the leading logarithmic approximation by $50 \%$. However, we cannot consider such an estimation as an accurate and reliable result. If the correction happens to have a value significant in comparison with the overall uncertainty, one has to improve the result. A use of the leading logarithmic terms is fruitful since they used to have some 'natural' values because they are always (or almost always) state-independent and not a result of big cancellations. The latter happens seldom between different gauge-invariant sets (e.g., in the $\alpha E_{F}$ term for the positronium hyperfine 
splitting) and is easy to recognize. It is clear what to do in the case of such a cancellation; it is necessary to estimate the non-leading terms separately by a half-value of the related leading terms and then to sum these uncertainty contributions as an rms sum.

"State-independence" means that the contributions are proportional to the wave function at the origin squared $|\Psi(0)|^{2}$ or its first derivative $\left|\Psi(0) \Psi^{\prime}(0)\right|$, i.e., to $\delta_{l 0} / n^{3}$, with no other dependence on $n$. In particular, state-independent effects do not contribute to $\Delta(n)$ and $D_{21}$ considered in this paper. The nonleading terms, in contrast, depend on a state and involve sometimes substantial cancellations. They also may involve various numerical cancellations and their calculation can hardly improve the reliability of the estimation. However, their evaluation is necessary as a step in direction towards the completion of the calculation of the whole correction and to check whether the non-leading terms are of a reasonable value which sometimes may be enhanced ${ }^{12}$. We underline that it could be no rigorous proof for such an empirical estimation - it is barely a result of experience based on a high number of known logarithmic contributions. Such an approach is not much different from what experimentalists do to estimate possible systematic errors. The estimation is always a guess, hopefully a plausible one, and definitely a deeper understanding of the problem is helpful. Our motivation is not only application of the factor of $1 / 5$ as a parameter, but also an observation that most functions in physics are quite smooth. The leading logarithmic term for some value of $Z \alpha$, which may be even much below $1 / 137$ related to a non-physical value of $Z \ll 1$, determines the sign and the magnitude of the correction. In such a case even if the parameter approaches unity, the order of magnitude is often still determined by the leading asymptotics as long as the function is smooth enough. Most of such smooth functions seldom change their sign and that is also important for the motivation of our method. A weak point of this explanation is that we already deal with certain higher order corrections, i.e., with a function with its first few terms of expansion subtracted. Even if the function itself is a "physical' one with the expected smooth behavior, it is not quite clear how smooth it should be after the subtractions. Also, we know that some smooth functions change sign etc. and in this case the asymptotics may be not helpful.

It seems that we have reached a certain numerical limit related to the logarithmic contribution and the calculation of the non-logarithmic terms will be much more complicated than anything else done before. However, it is strongly needed to improve the reliability and the accuracy of the theoretical

\footnotetext{
$\overline{12}$ In particular, large coefficients were discovered for linear logarithmic term for the Lamb shift $[67,68]$. The coefficients are so large that the term linear in $\ln (1 /(Z \alpha))$ dominates over a cubic logarithmic term. The result has not yet been confirmed by an independent group. Neither origin of the large numerical value of the coefficient is understood, nor possible consequences for estimation of other higher-order terms.
} 
predictions.

\subsection{Crucial 'soft QED' corrections}

Not only the crucial orders of magnitude offer a cross comparison of efficiency of different experiments. The modern bound state QED theory clearly recognizes two kinds of contributions: soft-photon contributions and hard-photon contributions. The latter are very similar to free QED, while the former essentially involve binding effects. There are two crucial soft-QED contributions.

- One of crucial binding corrections is due to the higher order of the one-loop and two-loop self-energy. The one-loop self energy was recently successfully calculated for most of important applications. The two-loop self-energy terms (see, e.g., Fig. 35) are partly known and uncertainty of this knowledge determines the accuracy of the Lamb shift calculations for hydrogen, helium ion, the medium- and high- $Z$ Lamb shift and fine structure, while similar diagrams with a transverse photon contribute to an uncertainty of $D_{21}$ in the helium-3 ion.

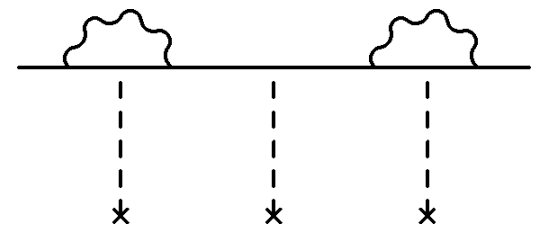

Fig. 35. One of diagrams for higher-order two-loop corrections (order of $\left.\alpha^{2}(Z \alpha)^{6} m c^{2}\right)$ to the Lamb shift. The logarithmic part contains up to the cube of the logarithm.

- The other important corrections are caused by recoil effects (see Figs. 36 and 37) and they determine the uncertainty of the muonium HFS, the positronium energy levels (the hyperfine interval, the $1 s-2 s$ transition, and the fine structure) and a part of the uncertainty of the $D_{21}$ difference for the helium-3 ion. The soft-photon nature of these contributions is clearly seen from the appearance of the bound logarithm $\ln (1 /(Z \alpha))$. Actually this logarithm often (but not always) arises as $\ln \left(m c^{2} /\langle E\rangle\right)$, where $\langle E\rangle$ is a characteristic atomic energy. The other possibility for this logarithm to appear is a logarithmic integration over coordinate or momentum space which leads to $\ln (m c\langle r\rangle / \hbar)$, where $\langle r\rangle$ is the characteristic size of the atomic state. Thus, the space-logarithm contributions contain a soft part with integrations over atomic momenta $k \sim(Z \alpha) m c$ and hard part related to integration over $k \sim m c$. In energy-logarithm cases, two low-momentum regions $\left(k \sim(Z \alpha)^{2} m c\right.$ and $\left.k \sim(Z \alpha) m c\right)$ can be distinguished.

If one calculates separately the soft- or hard-momentum contributions, the logarithm of an effective energy or momentum appears as a logarithm of a 
certain cut-off. That greatly simplifies evaluation of logarithmic corrections. Meantime, the appearance of such logarithm means that both the soft and hard contributions are divergent and only their sum have sense.

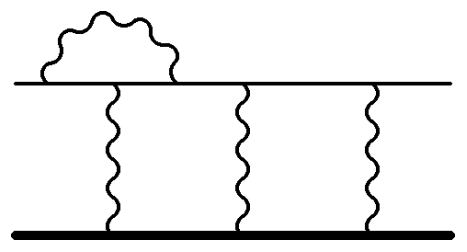

Fig. 36. An example of the diagrams for higher-order radiative recoil corrections to the HFS interval (relative order of $\alpha(Z \alpha)^{2} m / M$ ). The logarithmic part contains up to the square of the logarithm.

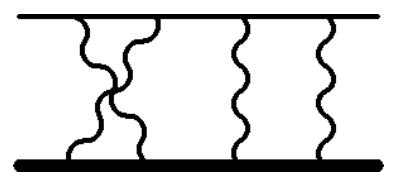

Fig. 37. An example of the diagrams for higher-order pure recoil corrections to the HFS interval (relative order of $(Z \alpha)^{3} \mathrm{~m} / M$ ). The logarithmic part contains double logarithmic contributions.

\subsection{Crucial 'hard QED' corrections}

The hard-photon effects are very similar to effects of free QED, however, the areas of integrations over momentum space are different. Both the free QED and 'hard' contributions to the bound-state QED suffer from infrared divergencies. This problem is also solved in different ways.

Most accurate free QED calculations which may be compared with experiment are related to the anomalous magnetic moments of an electron and a muon. The integration for them is performed over kind of an isotropic area in the Euclidean 4D space ${ }^{13}$, and the crucial level is a four-loop approximation. For the bound problems there are two other specific areas of integration.

- For some problems it is sufficient to apply an external-field approximation to hard-photon corrections, in which the exchange photons carry zero energy transfer (see, e.g., a diagram in Fig. 35). Even for recoil effects, the

\footnotetext{
$\overline{13}$ The physical expressions to calculate originate indeed from a description related to the Minkowsky space with the metrics $(1,-1,-1,-1)$, where, e.g., $k_{\mu} k^{\mu}=k_{0}^{2}-\mathbf{k}^{2}$. However, while calculating the diagrams it is often convenient to make the Wick rotation of the integration contour and to arrive at the Euclidian metric $(1,1,1,1)$ (or rather $(-1,-1,-1,-1)$ ).
} 
integration is essentially not covariant in the Euclidian 4D space. The highest crucial orders are related to four-loop corrections for the external-field and three-loop corrections for recoil effects (see, e.g., diagrams in Figs. 36 and 37). Still, for certain four-dimensional Euclidian variable one can observe for characteristic momenta $\left|k_{0}\right| \ll\left|k_{i}\right|$. Note that in contrast to the anomalous magnetic moment, this is a calculation for two different particles and thus it has its own simplifications and difficulties.

- The other specific situation for integration is related to the positronium annihilation when some photons are real and thus their momentum satisfy the condition $k^{2}=k_{0}^{2}-\mathbf{k}^{2}=0$. Studies of the orthopositronium decay mode (see, e.g., Fig. 38) and a branching fraction for five-photon decay $[253,194]$ allow us to check calculations of four-loop corrections in such a non-isotropic area of integration. The accuracy of determination of the branching of four-photon decay of parapositronium $[190,191,192,193,194]$ is approaching a level where four-loop diagrams are important.

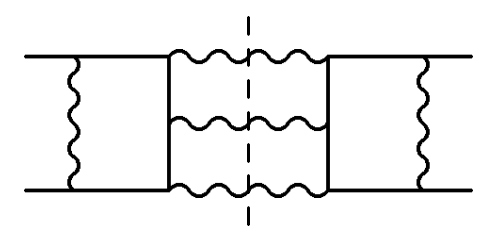

Fig. 38. An example of the diagrams for two-loop radiative corrections to the three-photon annihilation of orthopositronium).

The bound state problems supply us with an opportunity to check modern algorithms for four-loop calculations, competitive to the anomalous magnetic moment of an electron, and allow us to test them for different kinematic areas.

\section{What is next?}

Above, we have reviewed the present status on precision theory and experiment on light two-body atoms. In this section, we will briefly overview problems and possible experiments in the field.

The Lamb shift was successfully measured in hydrogen, deuterium and an ion of $\mathrm{He}^{+}$. Experiments in neutral atoms have reached a certain level of accuracy, which will be difficult to improve very much without completely new approaches. However, a certain progress is possible and is expected from optical frequency measurements.

Nevertheless, microwave experiments may be important. There remains a question about reliability of a microwave experiment by Sokolov and Yakovlev. Their results are reproducible within their experiment, which means that any 
problem related to possible systematic effects could be, in principle, resolved. Their statistical uncertainty is competitive with the present optical experiments. Unfortunately, the experiment was stopped. We hope it may be continued or reproduced.

We also note that a microwave study of hydrogen circular states at MIT is only a project that can deliver a really independent value of the Rydberg constant, but, unfortunately, the evaluation of the data remains unfinished.

In the case of the helium ion only microwave measurements have taken place. Ongoing optical experiments can offer essential progress.

Tritium is a light neutral hydrogen-like atom whose the Lamb shift has not yet been measured accurately. We are interested in tritium not because of QED, but mostly because we hope to learn more about its nuclear structure and in particular on its charge radius.

Uncertainty in determining the proton charge radius is the main obstacle in any improvement of the precision theory for the Lamb shift in hydrogen. The former scattering measurements were evaluated improperly, while their original data are not available. A new measurement, even with the same accuracy but with a transparent and reliable evaluation, would be very helpful. We can also learn the value of the proton charge radius by measuring the Lamb shift in muonic hydrogen; and we anticipate news from a related PSI experiment.

The hyperfine interval of the ground state in hydrogen has been known for a while with a high experimental accuracy. Any theoretical progress is possible there only with a better understanding of the proton structure. In particular it is crucial to have a reliable description of the proton polarizability effects. This may be done with improvement of the experimental data on scattering (muon-proton's or electron-proton's). We observed a certain interest by high-energy community to re-evaluate of various experimental data for the scattering (mainly due to determination of the proton charge radius). We hope for a reliable re-evaluation of existing data on inelastic scattering to be obtained soon. More data on spin-dependent inelastic muon-proton scattering are strongly needed.

An advanced phenomenological description of the nucleon structure and nucleonnucleon interaction, based on a constraint from experiment, is crucial not only for hyperfine splitting in hydrogen but also in few-nucleon light atoms, such as deuterium, tritium, helium-3 ion. Accuracy can be improved and we have observed some progress in this field. We hope that nuclear theory will help with prediction of the nuclear-structure contributions to the HFS interval for $A=3$ nucleus.

Successful comparison of the $1 s$ and $2 s$ hyperfine intervals is not completely 
sufficient. Accuracy of the experimental determination of the $2 s$ HFS interval in hydrogen and deuterium is still not high enough, however, its threefold improvement after applying optical methods allows to hope for future progress. The $2 s$ measurement in the ${ }^{3} \mathrm{He}^{+}$ion is still competitive with theory. However, it is not satisfactory when so important experimental value was never remeasured again. It seems that it is possible to go to somewhat higher $Z$ and study $\mathrm{Li}^{++}$ions.

The comparison of the $1 s$ and $2 s$ HFS intervals is not the only way to get rid of the nuclear effects. Another important option is spectroscopy of pure leptonic atoms, such as muonium and positronium. Unfortunately, this kind of experiments was completely stopped some time ago. There are no running or planned muonium projects, while all present positronium projects aim only at the study of decay. We think that a measurement of the $1 s$ HFS interval, $1 s-2 s$ transitions in muonium and positronium and $2 S-2 P$ fine structure intervals in positronium can deliver results quite competitive with theory. In the case of the hyperfine splitting in positronium the experiment is necessary also to resolve a discrepancy of approximately $3 \sigma$ which shows that our understanding of the situation is not completely satisfactory.

Successful experiments designed to determine the $g$ factor of a bound electron in a hydrogen-like ion with a spinless nucleus eventually led to the most accurate determination of the electron-to-proton mass ratio (or, which is essentially equivalent, of the electron mass in unified atomic mass units). The experiment was done with carbon-12 and oxygen-16. Improvement in accuracy is possible. It is also of crucial importance to present the data properly. A value $g\left({ }^{12} \mathrm{C}^{5+}\right) / g\left({ }^{12} \mathrm{O}^{7+}\right)$ should be presented by experimentalists by taking into account correlations between two measurements. The range of ions should be broadened to include ions from ${ }^{10} \mathrm{Be}^{3+}$ to ${ }^{40} \mathrm{Ca}^{19+}$. Medium- $Z$ lithium-like ions may be helpful at least to study systematic effects since they broaden a possible charge-to-mass ratio of ions and can be reasonably well understood theoretically.

These experiments are more accurate than the MIT study of Breit-Rabi levels in hydrogen and deuterium which delivered the best values of $\mu_{p} / \mu_{e}$ and $\mu_{d} / \mu_{e}$. However, these experiments were never reproduced and that is not satisfactory because of the great importance of an accurate value of $\mu_{p} / \mu_{e}$ for QED tests and determination of fundamental constants. In principle a value of $\mu_{p} / \mu_{e}$ can be and should be determined independently.

One of possibilities can be a determination of the anomalous magnetic moment ${ }^{14}$ of the deuteron (similarly to a measurement of the anomalous mag-

\footnotetext{
${ }^{14}$ We follow the common notation which is somewhat misleading. What is called 'anomalous magnetic moment' is rather 'anomalous $g$ factor'. In this paragraph we are discussing the anomalous contribution to the magnetic moment.
} 
netic moment of muon at the Brookhaven National Laboratory). That measurement was done for a value of $a_{\mu} \cdot\left(e \hbar / m_{\mu}\right) / \mu_{p}$. A similar result for a deuteron would be very helpful since the deuteron anomalous magnetic moment is small $\left(a_{d} \simeq-0.143\right)$ and its 'normal' value, related to the $g$ factor $g_{d}^{(0)}=2$, i.e., $g_{d}^{(0)} \cdot\left(e \hbar / 2 m_{d}\right)$, in units of the nuclear magneton is well known (that is simply the mass ratio for a proton and a deuteron multiplied by two). Smallness of the magnetic moment provides an enhancement of accuracy in determination of the magnetic moments

$$
\frac{\mu_{d}}{\mu_{N}}=\frac{2 m_{p}}{m_{d}}+\frac{2 a_{d} \cdot\left(e \hbar / 2 m_{d}\right)}{\mu_{p}} \cdot \frac{\mu_{p}}{\mu_{N}} .
$$

Comparing the deuteron and proton magnetic moments via NMR spectroscopy of HD molecules, one can find $g_{p}$.

Determination of the $g$ factor of a proton $\left(g_{p}=\mu_{p} / \mu_{N}\right)$ is essentially the same as a determination of $\mu_{p} / \mu_{e}$, since the conversion factor $\mu_{N} / \mu_{e}$ is known well enough. W. Quint suggested [254] to measure the proton $g$ factor directly using technics developed for the experiment on hydrogen-like ions with spinless nuclei.

The bound state QED theory is mainly satisfactory for hydrogen-like atoms for comparison with various present-day experiments. Taking into account future experiments, it is necessary to improve its accuracy calculating higherorder corrections. As one of the crucial problems, let us mention numerical and analytic calculations of one-loop contributions for the hyperfine difference $D_{21}$, two-loop corrections for the Lamb shift, $D_{21}$ and bound electron $g$ factor and various fourth-order contributions to the positronium energy levels, the muonium $1 s$ hyperfine interval and $D_{21}$.

This review is focussed on light atoms which offer various precision tests of bound state QED. "High precision" is understood as a high relative precision of various measurements and calculations and sometimes as a high sensitivity to certain high-order QED corrections. Certain study of medium- and high- $Z$ ions can be also of interest for QED tests. However, it is crucially important to clarify uncertainty of the corrections due to the nuclear charge distribution.

We note also that the high precision is not the only quantity of interest. One of the motivations to 'test' QED is a search for its violations; another is a test of various approaches for precision calculations and measurements. It is not necessary that the most precise tests are the most sensitive to any open questions. The sensitivity is often quite selective and depends on a problem under question. Study of tests related to any particular problem needs a separate study and other experiments may be important. 


\section{Summary}

When they said, "Does it fit?"

He replied, "Not a bit!"

Edward Lear

The situation concerning precision studies of simple atoms is fortunately opposite to that in the quoted verse by Lear. The theory and experiment agree well in general. There may be some discrepancy in a certain particular case, even a long standing one, but we have no reason to doubt quantum electrodynamics in its basic moments. However, the theory of simple atoms is a bound state QED theory and the crucial question is not whether QED proper is correct. The question is how successfully QED can be applied and has been applied to the bound states. The latter is not a simple issue and progress in QED calculations can be very fruitful for better understanding of the bound states in nuclear and particle physics. In early time of quantum physics theoretical progress established new theories and was of great significance. Now the most important part of physics of simple atoms lies in experiment.

Fascinating experimental progress with high-precision measurements, by bridging microwave, infrared, optical and in part ultraviolet domains of spectra, with dramatic cooling of atoms, with access to trapped single atoms and particles, with producing muonic and exotic atoms and various highly charged ions offers us a broad range of applications. However, studying simple objects with predictable properties is still the most natural choice. That is how simple atoms enter modern physics, most of progress of which is now rather a technological one and that is how simple atoms can serve for quite practical applications testing new experimental methods. Another application of simple atoms of practical importance is the determination of certain fundamental constants. These constants have been more and more involved in the modern system of units and standards.

Can we learn from simple atoms any really new physics? The answer for most experiments is rather negative. They have pursued other purposes. Simplicity of the atoms and reliability of the QED predictions make them a powerful tool to study effects beyond atomic physics, such as particle and nuclear properties. Precision study of two-body atoms delivers us information on structure of light nuclei (deuteron, triton, helion, and alpha particle), the structure of a proton, various properties of muons, pions and other particles. In general, we check consistency of QED theory and experiment and as long as we see the consistency, the constraints on new physics are related to the uncertainty of the experiment and theory. There are still some projects with simple atoms which are designed to look beyond the established physics: a search for exotic decay of positronium, a search for variations of the fundamental constants, a search for CPT violation studying properties of antihydrogen, a conversion of 
muonium into antimuonium etc.

Twenty years ago, when I joined the QED team at Mendeleev Institute for Metrology and started to work on theory of simple atoms, the accuracy of the experimental data for most QED tests was substantially higher than that of the theoretical predictions. Work of several theoretical groups from around the world during this twenty-year period has put theory of two-body atomic systems to a dominant position and we are now looking forward to obtaining new experimental results. Except the case of the $2 s$ hyperfine interval in the ${ }^{3} \mathrm{He}^{+}$ion, theory is more accurate than experiment. However, theory is still not perfect. For some quantities the gap between the theoretical and experimental accuracy is not big and we should not think that the theory is above any doubts. For example, starting my work on the review, I believed that the theory for the $D_{21}$ difference in helium-3 ion is more accurate than the experimental data, but a conservative re-estimation of the theoretical uncertainty $[115,116]$ has reversed the situation.

Several experiments are in progress or planned and substantial experimental progress is possible. Since the theoretical improvement needs some long-term programs, we have to start working on a further development of theory now. It will be indeed helpful for difficult theoretical calculations to be motivated by new experimental projects and new experimental results and I hope that both will follow.

The obvious success in one-electron atoms turned attention of some theorists to three-body (such as helium, muonic helium, antiprotonic helium) and fourbody systems and we hope that in some future it will be possible to state that theory of three- or maybe even four-body atoms has also superseded experiment.

\section{Acknowledgements}

I am grateful to G. W. F. Drake, S. I. Eidelman, J. Friar, T. W. Hänsch, E. Hinds, V. G. Ivanov, K. Jungmann, A. I. Milstein, P. Mohr, L. B. Okun, M. H. Prior, J. R. Sapirstein, V. A. Shelyuto, I. Sick, B. Taylor and G. Werth for useful and stimulating discussions. This work was supported in part by RFBR (grants \#\# 02-02-07027, 03-02-16843, 03-02-04029) and DFG (grant GZ 436 RUS 113/769/0-1). 


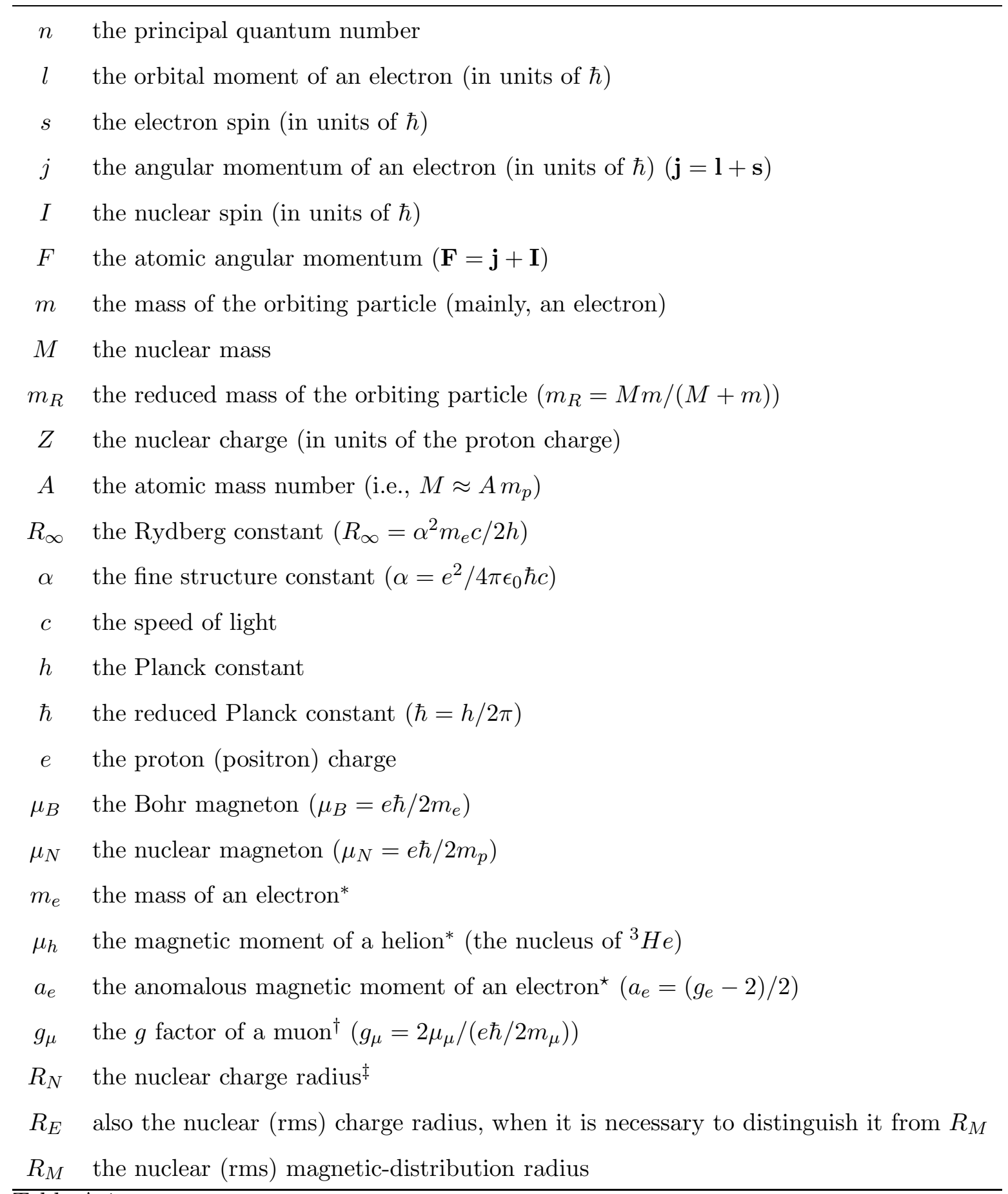

Table A.1

The most frequently used notation.

${ }^{*}$ - similar for other particles and nuclei; ${ }^{\star}$ - similar for a muon ${ }^{\dagger}{ }^{\dagger}-$ similar for an electron and a proton; ${ }^{\ddagger}$ - similar for a proton, a deuteron etc. 


\section{A Notations}

Unless otherwise stated, we use SI units. However, all numerical results for energy $E$ are presented in frequency units $E / h$.

Energy levels in a single-electron atom are labelled as $n l_{j}$ or if the hyperfine effects are involved as $n l_{j}(F)$. The orbital momentum is presented by letters $s$ for $l=0, p$ for for $l=1, d$ for $l=2, f$ for $l=3$ etc. For few-electron systems (and positronium) the capital letters for angular momenta are used such as $n^{\kappa} L_{J}$ where $\kappa=2 S+1$.

\section{B Extract from the list of the recommended fundamental con- stants (CODATA, 2002)}

Here, we summarize the most recent CODATA recommended values of fundamental constants related to QED. We need to emphasize that Table B.1 contains results obtained in the $2002^{15}$ adjustment of the fundamental constants [23], while through out the paper we compare original results with the older set of recommended values [21]. A reason for that is that a substantial part of results reviewed here was obtained after 1998 and was not included in the earlier adjustment [21] and thus comparing the results we clearly see the recent progress in the field. The recent adjustment [23] already included nearly all original results reviewed in this work.

\footnotetext{
${ }^{15}$ We remind that 1998 and 2002 are years of the deadline for collecting the data for the evaluation.
} 


\begin{tabular}{lc}
\hline Constant & CODATA, \\
& $2002[23]$ \\
\hline$R_{\infty}$ & $10973731.568525(73) \mathrm{m}^{-1}$ \\
$c R_{\infty}$ & $3.289841960360(22) \times 10^{15} \mathrm{~Hz}$ \\
$R_{H}$ & $10967758.340642(73) \mathrm{m}^{-1 \star}$ \\
$\alpha^{-1}$ & $137.03599911(46)$ \\
$m_{e}$ & $5.4857990945(24) \times 10^{-4} \mathrm{u}$ \\
$a_{e}$ & $1.1596521859(38) \times 10^{-3}$ \\
$m_{p}$ & $1.00727646688(13) \mathrm{u}$ \\
$m_{p} / m_{e}$ & $1836.15267261(85)$ \\
$\mu_{p} / \mu_{B}$ & $1.521032206(15) \times 10^{-3}$ \\
$g_{p}$ & $5.585694701(56)$ \\
$\mu_{d} / \mu_{B}$ & $0.4669754567(50) \cdot 10^{-3}$ \\
$g_{d}$ & $0.8574382329(92)$ \\
$m_{\mu}$ & $0.1134289264(30) \mathrm{u}$ \\
$m_{\mu} / m_{e}$ & $206.7682838(54)$ \\
$\mu_{\mu} / \mu_{p}$ & $3.183345118(89)^{*}$ \\
$a_{\mu}$ & $1.16591981(62) \times 10^{-3}$ \\
\hline
\end{tabular}

Table B.1

New recommended values of the fundamental constants (CODATA, 2002, [23]) related to atomic physics and QED.

* The result for the Rydberg constant for the hydrogen atom $R_{H}$ was not presented in [23] directly and it is derived from values for $R_{\infty}$ and $m_{e} / m_{p}{ }^{*}$ We ignore in our paper (except of consideration of $D_{21}$ ) the negative sign of the ratio of the magnetic moments.

\section{References}

[1] J. E. Nafe, E. B. Nelson and I. I. Rabi, Phys. Rev. 71, 914 (1947).

[2] J. S. Rigden. Hydrogen: The Essential Element (Harvard University Press, 2002).

[3] A. Gubmeridze, Th. Stölker, D. Banaś, K. Beckert, P. Beller, H. F. Beyer, F. Bosch, S. Hagmann, C. Kozhuharov, D. Liesen, F. Nolden, X. Ma, P. H. Mokler, M. Steck, D. Sierpowski, and S. Tashenov, Phys. Rev. Lett. 94, 223001 (2005).

[4] V. A. Yerokhin and V. M. Shabaev, Phys. Rev. A64, 062507 (2001). 
[5] T. Franosch and G. Soff, Z. Phys. 18, 219 (1991).

[6] P. J. Mohr, G. Plunien, and G. Soff, Phys. Rep. 293, 227 (1998).

[7] V.A. Yerokhin, A. N. Artemyev and V. M. Shabaev, Phys. Lett. A234, 361 (1997).

[8] J.D. Zumbro, E. B. Shera, Y. Tanaka, C. E. Bemis, Jr., R. A. Naumann, M. V. Hoehn, W. Reuter, and R. M. Steffen, Phys. Rev. Lett. 53, 1888 (1984).

[9] H. A. Bethe and E. E. Salpeter, Quantum Mechanics of One- and Two-electon Atoms (Plenum, NY, 1977).

[10] G. W. Series, The Spectrum of Atomic Hydrogen: Advances (World Sci., Singapore, 1988).

[11] B. E. Lautrup, A. Peterman, and E. de Rafael, Phys. Rep. 3, 193 (1972).

[12] T. Kinoshita (Ed.), Quantum Electrodynamics (World Sci., Singapore, 1990).

[13] S. G. Karshenboim, F. S. Pavone, F. Bassani, M. Inguscio and T. W. Hänsch (Eds.), Hydrogen atom: Precision physics of simple atomic systems (Springer, Berlin, Heidelberg, 2001).

[14] S. G. Karshenboim and V. B. Smirnov (Eds.), Precision physics of simple atomic systems (Springer, Berlin, Heidelberg, 2003).

[15] J. Sapirstein and D. R. Yennie, In [12], p. 560.

[16] M. I. Eides, H. Grotch and V. A Shelyuto, Phys. Rep. 342, 63 (2001).

[17] G. F. Bassani, M. Inguscio and T. W. Hänsch (Eds.), The Hydrogen Atom, Proceedings of the Simposium, Held in Pisa, Italy June, 30-July, 2, 1988. (Springer-Verlag, Berlin, Heidelberg, 1989). Presented also in CD'part of Ref. [13].

[18] The International Conference on the Precision Physics of Simple Atomic Systems (PSAS 2002). Proceedings. Can. J. Phys. 80-11, pp. 1187-1432 (2002).

[19] The International Conference on the Precision Physics of Simple Atomic Systems (PSAS 2004). Proceedings. Can. J. Phys. 83-4, pp. 273-474 (2005).

[20] E. R. Cohen and B. N. Taylor, Rev. Mod. Phys. 59, 1121 (1987).

[21] P. J. Mohr and B. N. Taylor, Rev. Mod. Phys. 72, 351 (2000).

[22] P. J. Mohr and B. N. Taylor, In Ref. [13], p. 145.

[23] P. J. Mohr and B. N. Taylor, 77, 1 (2005).

[24] S. G. Karshenboim, Physics-Uspekhi, 48, 255 (2005).

[25] See, e.g., E. G. Myers, In Ref. [13], p. 179.

[26] T. Stöhlker, D. Banaś, H. Beyer and A. Gumberidze, In Ref. [14], p. 115. 
[27] L. Nemenov, In Ref. [13], p. 223.

[28] T. Yamazaki, In Ref. [13], p. 246;

T. Yamazaki, N. Morita, R. S. Hayano, E. Widmann and J. Eades, Phys. Rep. 366, 183 (2002).

[29] T. S. Jensen and V. E. Markushin, In Ref. [14], p. 37;

V.E. Markushin, Can. J. Phys. 80, 1271 (2002).

[30] T. Yamazaki, In Ref. [14], p. 79

[31] E. A. Hinds, In [10], p. 243.

[32] F. M. Pipkin, In [12], p. 479.

[33] F. Biraben, T.W. Hänsch, M. Fischer, M. Niering, R. Holzwarth, J. Reichert, Th. Udem, M. Weitz, B. de Beauvoir, C. Schwob, L. Jozefowski, L. Hilico, F. Nez, L. Julien, O. Acef, J.-J. Zondy, and A. Clairon, In Ref. [13], p. 17.

[34] S. G. Karshenboim, JETP 79, 230 (1994).

[35] S. G. Karshenboim, Z. Phys. D39, 109 (1997).

[36] S. G. Karshenboim, Can. J. Phys. 77, 241 (1999).

[37] J. Ch. De Vries, Ph. D. Thesis, MIT, 2001.

[38] T. Udem, A. Huber, B. Gross, J. Reichert, M. Prevedelli, M. Weitz and T. W. Hänsch, Phys. Rev. Lett. 79, 2646 (1997);

M. Niering, R. Holzwarth, J. Reichert, P. Pokasov, Th. Udem, M. Weitz, T. W. Hänsch, P. Lemonde, G. Santarelli, M. Abgrall, P. Laurent, C. Salomon, and A. Clairon, Phys. Rev. Lett. 84, 5496 (2000).

[39] R. de Beauvoir, F. Nez, B. cagnac, F. Biraben, D. Touahri, L. Hilico, O. Acef, A. Clairon and J. J. Zondy, Phys. Rev. Lett. 78, 440 (1997);

C. Schwob, L. Jozefowski, B. de Beauvoir, L. Hilico, F. Nez, L. Julien and F. Biraben, Phys. Rev. Lett. 82, 4960 (1999).

[40] W. E. Lamb, Jr., and R. C. Retherford, Phys. Rev. 72, 241 (1947); Phys. Rev. 75, 1325 (1949); Phys. Rev. 79, 549 (1950).

[41] M. Skinner and W. E. Lamb, Jr., Phys. Rev. 75, 1325 (1949); Phys. Rev. 78, 539 (1950).

[42] S. R. Lundeen and F. M. Pipkin, Phys. Rev. Lett. 46, 232 (1981); Metrologia 22, 9 (1986).

[43] A. van Wijngaarden, F. Holuj and G. W. F. Drake, Can. J. Phys. 76, 95 (1998).

[44] D. A. Andrews and G. Newton. Phys. Rev. Lett. 37, 1254 (1976); G. Newton, D. A. Andrews and P. J. Unsworth, Philos. Trans. Roy. Soc. London 290, 373 (1979);

R. T. Robiscoe and T. W. Shyn, Phys. Rev. Lett. 24, 559 (1970). 
[45] Y. L. Sokolov and V. P. Yakovlev, Sov. Phys. JETP 56, 7 (1982);

V. G. Pal'chikov, Yu. L. Sokolov, V. P. Yakovlev, JETP Lett. 38, 418 (1983);

Metrologia 21, 99 (1985).

[46] S. G. Karshenboim, Phys. Scripta 57, 213 (1998).

[47] E. W. Hagley and F. M. Pipkin, Phys. Rev. Lett. 72, 1172 (1994).

[48] B. L. Cosens and T. V. Vorburger, Phys. Rev. Lett. 23 (1969) 1273; Phys. Rev. A2, 16 (1970);

T. W. Shyn, T. Rebane, R. T. Robiscoe and W. L. Williams, Phys. Rev. A3, 116 (1971);

K. A. Safinya, K. K. Chan, S. R. Lundeen and F. M. Pipkin, Phys. Rev. Lett. 45, 1934 (1980) (the result is corrected accordingly to [47]).

[49] S. J. Brodsky and R. G. Parsons, Phys. Rev. 163, 134 (1967); 176, 423E (1968).

[50] K. Pachucki, Phys. Rev. A53, 2092 (1996).

[51] S. G. Karshenboim, Phys. At. Nucl. 58, 835 (1995); JETP 80, 593 (1995);

V. G. Ivanov and S. G. Karshenboim, Phys. Lett. A210, 313 (1996).

[52] V. G. Ivanov and S. G. Karshenboim, JETP 82, 656 (1996); 84, 209(E) (1997).

[53] J. Sapirstein, K. Pachucki and K.T. Cheng, Phys. Rev. A69, 022113 (2004).

[54] A. Huber, Th. Udem, B. Gross, J. Reichert, M. Kourogi, K. Pachucki, M. Weitz and T. W. Hänsch, Phys. Rev. Lett. 80, 468 (1998).

[55] M. Weitz, A. Huber, F. Schmidt-Kaler, D. Leibfried, W. Vassen, C. Zimmermann, K. Pachucki, T. W. Hänsch, L. Julien, and F. Biraben, Phys. Rev. A52, 2664 (1995).

[56] M. Weitz, F. Schmidt-Kaler, and T. W. Hänsch, Phys. Rev. Lett. 68, 1120 (1992); M. Weitz, A. Huber, F. Schmidt-Kaler, D. Leibfried, and T. W. Hänsch, Phys. Rev. Lett. 72, 328 (1994).

[57] D. J. Berkeland, E. A. Hinds and M. G. Boshier, Phys. Rev. Lett. 75, 2470 (1995).

[58] S. Bourzeix, B. de Beauvoir, F. Nez, M. D. Plimmer, F. de Tomasi, L. Julien, F. Biraben and D. N. Stacey, Phys. Rev. Lett. 76, 384 (1996).

[59] J. Reichert, M. Niering, R. Holzwarth, M. Weitz, Th. Udem, and T. W. Hänsch, Phys. Rev. Lett. 84, 3232 (2000);

R. Holzwarth, Th. Udem, T. W. Hänsch, J. C. Knight, W. J. Wadsworth, and P. St. J. Russell, Phys. Rev. Lett. 85, 2264 (2000);

S. A. Diddams, D. J. Jones, J. Ye, S. T. Cundiff, J. L. Hall, J. K. Ranka, R. S. Windeler, R. Holzwarth, Th. Udem, and T. W. Hänsch, Phys. Rev. Lett. 84, 5102 (2000).

[60] S. G. Karshenboim, In Atomic Physics 17 (AIP conference proceedings 551) Ed. by E. Arimondo et al. (AIP, 2001), p. 238. 
[61] S. G. Karshenboim, JETP 76, 541 (1993).

[62] U. D. Jentschura, P. J. Mohr, and G. Soff, Phys. Rev. Lett. 82, 53 (1999).

[63] A. N. Artemyev, V. M. Shabaev, and V. A. Yerokhin, Phys. Rev. A52, 1884 (1995).

[64] K. Pachucki, Ann. Phys. (N.Y.) 226, 1 (1993).

[65] K. Pachucki, Phys. Rev. Lett. 72, 3154 (1994).

[66] M. Eides and V. Shelyuto, JETP Letters 61, 478 (1995); Phys. Rev. A52, 954 (1995).

[67] K. Pachucki, Phys. Rev. A63, 042053 (2001).

[68] K. Pachucki and U. D. Jentschura, Phys. Rev. Lett. 91, 113005, (2003);

U. D. Jentschura and I. Nandori, Phys. Rev. A66, 022114 (2002);

U. D. Jentschura and K. Pachucki, J. Phys. A35, 1927 (2002);

U. D. Jentschura, Phys. Rev. A70, 052108 (2004).

[69] V. A. Yerokhin, P. Indelicato and V. M. Shabaev, Phys. Rev. A71, 040101 (R) (2005).

[70] K. Melnikov and T. V. Ritbergen, Phys. Rev. Lett. 84, 1673 (2000).

[71] K. Pachucki and H. Grotch, Phys. Rev. A51, 1854 (1995).

[72] M. I. Eides and H. Grotch, Phys. Rev. A55, 3351 (1997).

[73] K. Pachucki, Phys. Rev. A52, 1079 (1995).

[74] M. I. Eides, H. Grotch, and V. A. Shelyuto, Phys. Rev. A63, 052509 (2001).

[75] A. Czarnecki and K. Melnikov, Phys. Rev. Lett. 87, 013001. (2001).

[76] S. G. Karshenboim, Can. J. Phys. 76, 169 (1998); JETP 89, 850 (1999).

[77] K. Pachucki, private communicaton.

[78] M. I. Eides and V. A. Shelyuto, Phys. Rev. A68, 042106 (2003).

[79] L. Hand, D. I. Miller and R. Willson, Rev. Mod. Phys. 35, 335 (1963).

[80] G. G. Simon, Ch. Schmitt, F. Borkowski and V. H. Wather, Nucl. Phys. A333, 381 (1980).

[81] P. Lehmann, R. Taylor and R. Wilson, Phys. Rev. 126, 1183 (1962);

B. Dudelzak, G. Sauvage and P. Lehmann, Nuovo Cim. XXVIII, 18 (1963).

[82] J. J. Murphy, II, Y. M. Shin and D. M. Skopik, Phys. Rev. C9, 2125 (1974).

[83] Ch. W. Wong, Int. J. Mod. Phys. 3, 821 (1994).

[84] P. Mergell, U. G. Meissner and D. Drechsel, Nucl. Phys. A596, 367 (1996). 
[85] M. G. Boshier, In Atomic Physics 15, Ed. by H. B. van Linden van den Heuvell et al. (World Sci., Singapore, 1997), p. 328.

[86] R. Rosenfelder, Phys. Lett. B479, 381 (2000).

[87] I. Sick, Phys. Lett. B576, 62 (2003).

[88] R. Pohl, F. Biraben, C.A.N. Conde, C. Donche-Gay, T.W. Hänsch, F. J. Hartmann, P. Hauser, V. W. Hughes, O. Huot, P. Indelicato, P. Knowles, F. Kottmann, Y.-W. Liu, V. E. Markushin, F. Mulhauser, F. Nez, C. Petitjean, P. Rabinowitz, J.M.F. dos Santos, L. A. Schaller, H. Schneuwly, W. Schott, D. Taqqu, and J.F.C.A. Veloso, In Ref. [13], p. 454.

[89] A. van Wijngaarden, F. Holuj, and G. W. F. Drake, Phys. Rev. A63, 012505 (2000).

[90] J. L. Friar and G. L. Payne, Phys. Rev. C56, 619 (1997).

[91] J.L. Friar, in [14], p. 59, eprint nucl-th/0211064 Can. J. Phys. 80, 1337 (2002).

[92] H. Hellwig, R.F.C. Vessot, M. W. Levine, P. W. Zitzewitz, D. W. Allan, and D. J. Glaze, IEEE Trans. IM-19, 200 (1970).

[93] P. W. Zitzewitz, E. E. Uzgiris, and N. F. Ramsey, Rev. Sci. Instr. 41, 81 (1970).

[94] L. Essen, R. W. Donaldson, E.G. Hope and M. J. Bangham, Metrologia 9, 128 (1973).

[95] D. Morris, Metrologia 7, 162 (1971).

[96] V. S. Reinhard and J. Lavanceau, in Proceedings of the 28th Annual Symposium on Frequency Control (Fort Mammouth, N. J., 1974), p. 379.

[97] P. Petit, M. Desaintfuscien and C. Audoin, Metrologia 16, 7 (1980).

[98] J. Vanier and R. Larouche, Metrologia 14, 31 (1976).

[99] Y. M. Cheng, Y. L. Hua, C. B. Chen, J. H. Gao and W. Shen, IEEE Trans. IM-29, 316 (1980).

[100] A result from the Dhaanxi Astronomical Observatory, unpublished. Quoted accordingly to [99].

[101] S. G. Karshenboim, Can. J. Phys. 78, 639 (2000).

[102] L. Essen, R. W. Donaldson, E. G. Hope and M. J. Bangham, Nature 229, 110 (1971).

[103] D. J. Wineland and N. F. Ramsey, Phys. Rev. 5, 821 (1972).

[104] B. S. Mathur, S. B. Crampton, D. Kleppner and N. F. Ramsey, Phys. Rev. 158, 14 (1967).

[105] H. A. Schluessler, E. N. Forton and H. G. Dehmelt, Phys. Rev. 187, 5 (1969). 
[106] N. Kolachevsky, M. Fischer, S. G. Karshenboim and T.W. Hänsch, Phys. Rev. Lett. 92, 033003 (2004); M. Fischer, N. Kolachevsky, S. G. Karshenboim and T.W. Hänsch, Can. J. Phys. 80, 1225 (2002).

[107] N. E. Rothery and E. A. Hessels, Phys. Rev. A61, 044501 (2000).

[108] J. W. Heberle, H. A. Reich and P. Kush, Phys. Rev. 101, 612 (1956).

[109] N. Kolachevsky, P. Fendel, S.G. Karshenboim, and T.W. Hänsch, Phys. Rev. A, (2004); S.G. Karshenboim, P. Fendel, V. G. Ivanov, N. Kolachevsky and T.W. Hänsch, Can. J. Phys. 83, 283 (2005).

[110] H. A. Reich, J. W. Heberle, and P. Kush, Phys. Rev. 104, 1585 (1956).

[111] M. H. Prior and E.C. Wang, Phys. Rev. A16, 6 (1977).

[112] R. Novick and D. E. Commins, Phys. Rev. 111, 822 (1958).

[113] S. G. Karshenboim and V. G. Ivanov, Phys. Lett. B524, 259 (2002); Euro. Phys. J. D19, 13 (2002).

[114] S. G. Karshenboim, In Ref. [14], p. 141.

[115] S.G. Karshenboim, N. Kolachevsky, V. G. Ivanov, P. Fendel, M. Fischer, and T.W. Hänsch, JETP (2005) to be probably published (the paper has been accepted, however, because of a problem with technical editors its publication becomes questionable).

[116] S.G. Karshenboim and V. G. Ivanov, Can. J. Phys. (2005) to be published; eprint hep-ph/0507322

[117] S. D. Drell and F. Zachariansen. Elecromagnetic structure of nucleons. Oxford University Press (1961);

L. D. Landau and E. M. Lifshitz. Course of theoretical physics. Vol. 4: V. B. Bersetetskii, E. M. Lifshitz and L.P. Pitaevskii. Quantum electrodynamics. Pergamon press (Oxford, 1982).

[118] I. B. Khriplovich, A. I. Milstein, and S. S. Petrosyan, Phys. Lett. B366, 13 (1996), JETP 82, 616 (1996).

[119] J. L. Friar and I. Sick, Phys. Lett. B579, 285 (2004).

[120] G. Breit, Phys. Rev. 35, 1477 (1930).

[121] D. Zwanziger, Phys. Rev. 121, 1128 (1961).

[122] M. Sternheim, Phys. Rev. 130, 211 (1963).

[123] P. Mohr, unpublished. Quoted accordingly to [111].

[124] S. G. Karshenboim, In Ref. [13], p. 335.

[125] V. A. Yerokhin and V. M. Shabaev, Phys. Rev. A64, 012506 (2001).

[126] I. Sick, Progr. Part. Nucl. Phys. 55, 440 (2005). 
[127] I. Sick, Progr. Part. Nucl. Phys. 47, 245 (2001).

[128] A. H. Wapstra, G. Audi and C. Thibault, Nucl. Phys. A729, 129 (2003); G. Audi, A. H. Wapstra and C. Thibault, Nucl. Phys. A729, 337 (2003).

[129] Yu.I. Neronov and S.G. Karshenboim, Phys. Lett. A318, 126 (2003);

S. G. Karshenboim, V. G. Ivanov, Yu. I. Neronov, B. P. Nikolaev and Yu. N. Tolparov, Can. J. Phys. 83, 405 (2005).

[130] F. Maas, B. Braun, H. Geerds, K. Jungmann, B. E. Matthias, G. zu Putlitz, I. Reinhard, W. Schwarz, L. Williams, L. Zhang, P. E. G. Baird, P. G. H. Sandars, G. Woodman, G. H. Eaton, P. Matousek, T. Toner, M. Towrie, J. R. M. Barr, A. I. Ferguson, M. A. Persaud, E. Riis, D. Berkeland, M. Boshier and V. W. Hughes, Phys. Lett. A187, 247 (1994).

[131] W. Liu, M. G. Boshier, S. Dhawan, O. van Dyck, P. Egan, X. Fei, M. G. Perdekamp, V. W. Hughes, M. Janousch, K. Jungmann, D. Kawall, F. G. Mariam, C. Pillai, R. Prigl, G. zu Putlitz, I. Reinhard, W. Schwarz, P. A. Thompson, and K. A. Woodle, Phys. Rev. Lett. 82, 711 (1999).

[132] K. Jungmann, In Ref. [13], p. 81.

[133] A. Czarnecki, S. I. Eidelman and S. G. Karshenboim, Phys. Rev. D65, 053004 (2002).

[134] T. Kinoshita and M. Nio, Phys. Rev. Lett. 90, 021803 (2003).

[135] F. G. Mariam, W. Beer, P. R. Bolton, P. O. Egan, C. J. Gardner, V. W. Hughes, D. C. Lu, P. A. Souder, H. Orth, J. Vetter, U. Moser, and G. zu Putlitz, Phys. Rev. Lett. 49, 993 (1982).

[136] E. Klempt, R. Schulze, H. Wolf, M. Camani, F. Gygax, W. Rüegg, A. Schenck, and H. Schilling, Phys. Rev. D25, 652 (1982).

[137] G. W. Bennett, B. Bousquet, H. N. Brown, G. Bunce, R. M. Carey, P. Cushman, G. T. Danby, P. T. Debevec, M. Deile, H. Deng, W. Deninger, S. K. Dhawan, V. P. Druzhinin, L. Duong, E. Efstathiadis, F. J. M. Farley, G. V. Fedotovich, S. Giron, F. E. Gray, D. Grigoriev, M. Grosse-Perdekamp, A. Grossmann, M. F. Hare, D. W. Hertzog, X. Huang, V. W. Hughes, M. Iwasaki, K. Jungmann, D. Kawall, B. I. Khazin, J. Kindem, F. Krienen, I. Kronkvist, A. Lam, R. Larsen, Y. Y. Lee, I. Logashenko, R. McNabb, W. Meng, J. Mi, J. P. Miller, W. M. Morse, D. Nikas, C. J. G. Onderwater, Y. Orlov, C. S. Özben, J. M. Paley, Q. Peng, C. C. Polly, J. Pretz, R. Prigl, G. zu Putlitz, T. Qian, S. I. Redin, O. Rind, B. L. Roberts, N. Ryskulov, P. Shagin, Y. K. Semertzidis, Yu. M. Shatunov, E. P. Sichtermann, E. Solodov, M. Sossong, A. Steinmetz, L. R. Sulak, A. Trofimov, D. Urner, P. von Walter, D. Warburton, and A. Yamamoto, Phys. Rev. Lett. 89, 101804 (2002);

S. Redin, R. M. Carey, E. Efstathiadis, M. F. Hare, X. Huang, F. Krinen, A. Lam, J. P. Miller, J. Paley, Q. Peng, O. Rind, B. L. Roberts, L. R. Sulak, A. Trofimov, G. W. Bennett, H. N. Brown, G. Bunce, G. T. Danby, R. Larsen, Y. Y. Lee, W. Meng, J. Mi, W. M. Morse, D. Nikas, C. Ozben, R. Prigl, Y. K. 
Semertzidis, D. Warburton, V. P. Druzhinin, G. V. Fedotovich, D. Grigoriev, B. I. Khazin, I. B. Logashenko, N. M. Ryskulov, Yu. M. Shatunov, E. P. Solodov, Yu. F. Orlov, D. Winn, A. Grossmann, K. Jungmann, G. zu Putlitz, P. von Walter, P. T. Debevec, W. Deninger, F. Gray, D. W. Hertzog, C. J. G. Onderwater, C. Polly, S. Sedykh, M. Sossong, D. Urner, A. Yamamoto, B. Bousquet, P. Cushman, L. Duong, S. Giron, J. Kindem, I. Kronkvist, R. McNabb, T. Qian, P. Shagin, C. Timmermans, D. Zimmerman, M. Iwasaki, M. Kawamura, M. Deile, H. Deng, S. K. Dhawan, F. J. M. Farley, M. GrossePerdekamp, V. W. Hughes, D. Kawall, J. Pretz, E. P. Sichtermann, and A. Steinmetz, Can. J. Phys. 80, 1355 (2002).

[138] K. Pachucki, Phys. Rev. A54, 1994 (1996).

[139] M. Nio and T. Kinoshita, Phys. Rev. D55, 7267 (1997).

[140] S. M. Schneider, W. Greiner, and G. Soff, Phys. Rev. A50, 118 (1994).

[141] T. Kinoshita and M. Nio, Phys. Rev. Lett. 72, 3803 (1994); Phys. Rev. D53, 4909 (1996).

[142] G. Bodwin, D. Yennie, and M. Gregorio, Phys. Rev. Lett. 48, 1799 (1982); Rev. Mod. Phys. 57, 723 (1985).

[143] J. R. Sapirstein, E. A. Terray, and D. R. Yennie, Phys. Rev. D29, 2290 (1984).

[144] M. Eides, S. Karshenboim, and V. Shelyuto, Phys. Lett. B177, 425 (1986); S. Karshenboim, V. Shelyuto, and M. Eides, Sov. Phys. JETP 65, 664 (1987); V. Brook, M. Eides, S. Karshenboim, and V. Shelyuto, Phys. Lett. B216, 401 (1989).

[145] M. Eides, S. Karshenboim, and V. Shelyuto, Phys. Lett. B202, 572 (1988); S. Karshenboim, V. Shelyuto, and M. Eides, Sov. Phys. JETP 67, 671 (1988).

[146] M. I. Eides, H. Grotch and V. A. Shelyuto, Phys. Rev. D65, 013003 (2002); D70, 07300 (2004).

[147] S. G. Karshenboim, in 25 th E.G.A.S. Conference. Abstracts. (Caen, 1993) P1010; in 1994 Conference on precision electromagnetic measurements. Digest. (Boulder, 1994) p. 225.

[148] S. G. Karshenboim, Z. Phys. D36, 11 (1996).

[149] K. Melnikov and A. Yelkhovsky, Phys. Rev. Lett. 86, 1498 (2001).

[150] R. Hill, Phys. Rev. Lett. 86, 3280 (2001).

[151] G. P. Lepage, Phys. Rev. A16, 863 (1977).

[152] G. T. Bodwin, D. R. Yennie and M. A. Gregorio, Phys. Rev. Lett. 41, 1088 (1978).

[153] K. Pachucki, unpublished. Quoted as presented at International Symposium on Lepton Moments (IWH Heidelberg, 1999) (http://www.physi.uni-heidelberg.de/ muon/lep/lep_proc/K_Pachucki/kp.html). 
[154] R. Arnowitt, Phys. Rev. 92, 1002 (1953);

W. A. Newcomb and E. E. Salpeter, Phys. Rev. 97, 1146 (1955).

[155] S. F. Blundell, K. T. Cheng and J. Sapirstein, Phys. Rev. Lett. 78, 4914 (1997).

[156] M. Nio, In Quantum Electrodynamics and Physics of the Vacuum, ed. by G. Cantatore (AIP Confenrece Proceedings 564, AIP, Melville, 2001), p. 178.

[157] S. G. Karshenboim, V. G. Ivanov and V. M. Shabaev, Phys. Sc. T80, 491 (1999).

[158] M. I. Eides, H. Grotch and V. A. Shelyuto, Phys. Rev. D58, 013008 (1998).

[159] M. I. Eides and V. A. Shelyuto, Phys. Lett. 146B, 241 (1984).

[160] M. I. Eides, S. G. Karshenboim and V. A. Shelyuto, Phys. Lett. B216, 405 (1989); S. G. Karshenboim, V. A. Shelyuto and M. I. Eides, Sov. J. Nucl. Phys. 49, 309 (1989).

[161] S. G. Karshenboim and V. A. Shelyuto, Phys. Lett. B517, 32 (2001).

[162] S. I. Eidelman, S. G. Karshenboim and V. A. Shelyuto, Can. J. Phys. 80, 1297 (2002).

[163] S. Geer, Phys. Rev. D57, 6989 (1998); D59, 039903 (E) (1998);

B. Autin, A. Blondel and J. Ellis (eds.), Prospective study of muon storage rings at CERN, Report CERN-99-02.

[164] A. Karimkhodzhaev and R. N. Faustov, Sov. J. Nucl. Phys. 53, 626 (1991).

[165] R. N. Faustov, A. Karimkhodzhaev and A. P. Martynenko, Phys. Rev. A59, 2498 (1999).

[166] R. S. Conti, R. S. Vallery, D. W. Gidley, J. J. Engbrecht, M. Skalsey, and P.W. Zitzewitz, In Ref. [13], p. 103.

[167] R. Ley and G. Werth, In Ref. [13], p. 407; R. Ley, Appl. Surf. Sci. 194, 301 (2002).

[168] S. G. Karshenboim, Appl. Surf. Sci. 194, 307 (2002).

[169] S. G. Karshenboim, Int. J. Mod. Phys. A19, 3879 (2004).

[170] M. W. Ritter, P. O. Egan, V. W. Hughes and K. A. Woodle, Phys. Rev. A30, 1331 (1984)

[171] A. P. Mills, Jr., and G. H. Bearman, Phys. Rev. Lett. 34, 246 (1975);

A. P. Mills, Jr., Phys. Rev. A27, 262 (1983)

[172] M. S. Fee, A. P. Mills, Jr., S. Chu, E. D. Shaw, K. Danzmann, R. J. Chicherster and D. M. Zuckerman, Phys. Rev. Lett. 70, 1397 (1993)

[173] K. Danzmann, M. Fee, and S. Chu, Phys. Rev. A39, 6072 (1989). 
[174] K. Pachucki and S. G. Karshenboim, Phys. Rev. Lett. 80, 2101 (1998).

[175] K. Pachucki and S. G. Karshenboim, Phys. Rev. A60, 2792 (1999).

[176] T. C. Griffith, G. R. Heyland, K. S. Lines and T. R. Twomey, J. Phys. B11, L743 (1978).

[177] P. Hasbach, G. Hilkert, E. Klempt and G. Werth, Nuovo Cim. A97, 419 (1987).

[178] C. I. Westbrook, D. W. Gidley, R. S. Conti, and A. Rich, Phys. Rev. A40, 5489 (1989).

[179] D. Gidley, private communication.

[180] J.S. Nico, D.W. Gidley, A. Rich, and P.W. Zitzewitz, Phys. Rev. Lett. 65, 1344 (1990).

[181] S. Asai, S. Orito, and N. Shinohara, Phys. Lett. B357, 475 (1995).

[182] R. S. Vallery, P. W. Zitzewitz, and D. W. Gidley, Phys. Rev. Lett. 90, 203402 (2003)

[183] O. Jinnouchi, S. Asai and T. Kobayashi, Intl. J. Mod. Phys. A19, 3927 (2004).

[184] G. S. Adkins, R. N. Fell, and P. Mitrikov, Phys. Rev. Lett. 79, 3383 (1997);

A. H. Hoang, P. Labelle, and S. M. Zebarjad, Phys. Rev. Lett. 79, 3387 (1997).

[185] B. Kniehl and A. A. Penin, Phys. Rev. Lett. 85, 5094 (2000).

[186] G. S. Adkins, R. N. Fell, and J. Sapirstein, Phys. Rev. Lett. 84, 5086 (2000); Phys. Rev. A63, 032511 (1999).

[187] A. Czarnecki, K. Melnikov, and A. Yelkhovsky, Phys. Rev. Lett. 83, 1135 (1999).

[188] K. Melnikov and A. Yelkhovsky, Phys. Rev. D62, 116003 (2000); B. Khiehl and A. A. Penin, Phys. Rev. Lett. 85, 1210; 3065 (E) (2000).

[189] A.H. Al-Ramadhan and D.W. Gidley, Phys. Rev. Lett. 72, 1632 (1994).

[190] S. Adachi, M. Chiba, T. Hirose, S. Nagayama, Y. Nakamitsu, T. Sato, and T. Yamada, Phys. Rev. Lett. 65, 2634 (1990).

[191] H. von Busch, P. Thirolf, Ch. Ender, D. Habs, F. Köck, Th. Schulze and D. Schwalm, Phys. Lett. B325, 300 (1994).

[192] S. Adachi, M. Chiba, T. Hirose, S. Nagayama, Y. Nakamitsu, T. Sato, and T. Yamada, Phys. Rev. A49, 3201 (1994).

[193] J. Yang, M. Chiba, R. Hamatsu, T. Hirose, T. Matsumoto, and J. Yu, Phys. Rev. A54, 1952 (1996).

[194] P. A. Vetter and S. J. Freedman, Phys. Rev. A66, 052505 (2002). 
[195] A. P. Mills, Jr., S. Berko and K. F. Canter, Phys. Rev. Lett. 34, 1541 (1975);

S. Berko and H. N. Pendleton, Ann. Rev. Nucl. Part. Sci. 30, 453 (1980).

[196] S. Hatamian, R. S. Conti and A. Rich, Phys. Rev. Lett. 58, 1833 (1987).

[197] E. W. Hagena, R. Ley, D. Weil, G. Werth, W. Arnold and H. Schneider, Phys. Rev. Lett. 71, 2887 (1993).

[198] R. S. Conti, S.Hatamian, L. Lapidus, A. Rich and M. Skalsey, Phys. Lett. A177, 43 (1993).

[199] R. Ley, D. Hagena, D. Weil, G. Werth, W. Arnold and H. Schneider, Hyperfine Interactions 89, 327 (1994).

[200] K. Melnikov and A. Yelkhovsky, Phys. Lett. B458, 143 (1999).

[201] A. Czarnecki, K. Melnikov, and A. Yelkhovsky, Phys. Rev. Lett. 82, 311 (1999); Phys. Rev. A59, 4316 (1999).

[202] S. G. Karshenboim, In Ref. [13], p. 651.

[203] S. G. Karshenboim, Phys. Lett. A266, 380 (2000).

[204] G. Breit, Nature 122, 649 (1928).

[205] H. Grotch, Phys. Rev. Lett. 24, 39 (1970).

[206] R. N. Faustov, Nuovo Cim. 69A, 37 (1970); Phys. Lett. 33B, 422 (1970);

H. Grotch, Phys. Rev. A2, 1605 (1970);

F. E. Close and H. Osborn, Phys. Lett. 34B, 400 (1971);

H. Grotch and R. A. Hegstrom, Phys. Rev. A4, 59 (1971).

[207] S. G. Karshenboim and V. G. Ivanov, Can. J. Phys. 80, 1305 (2002).

[208] J. S. Tideman and H. G. Robinson, in Atomic Physics 7, Plenum Press, 1973, Ed. by S. J. Smith, G. K. Walters and L. H. Volsky, p. 85; Phys. Rev. Lett. 39, 602 (1977).

[209] W. M. Hughes and H. G. Robinson, Phys. Rev. Lett. 23, 1209 (1969); H. G. Robinson and W. M. Hughes, in Precision Measurement and Fundamental Constants, Washington, NBS spec. publ. 343, Ed. by D. N. Langenberg and B. N. Taylor, p. 427.

[210] F. G. Walther, W. D. Phillips and D. Kleppner, Phys. Rev. Lett. 28, 1159 (1972).

[211] D. J. Larson and N. F. Ramsey, Phys. Rev. A9, 1543 (1974).

[212] C. E. Johnson and H. G. Robinson, Phys. Rev. Lett. 45, 250 (1980).

[213] G. M. Keiser, H. G. Robinson and C. E. Johnson, Phys. Rev. Lett. 35, 1223 (1975); Phys. Rev. A16, 822 (1977).

[214] B. E. Zundell and V. W. Hughes, Phys. Lett. A59, 381 (1976). 
[215] C. W. White, W. M. Hughes, G. S. Hayne, and H. G. Robinson, Phys. Rev. 174, 23 (1968).

[216] M. I. Eides and H. Grotch, Ann. Phys. 260, 191 (1997).

[217] A. P. Martynenko and R. N. Faustov, JETP 93471 (2001).

[218] S. G. Karshenboim and V. G. Ivanov, Phys. Lett. B566, 27 (2003).

[219] P. F. Winkler, D. Kleppner, T. Myint and F. G. Walther, Phys. Rev. A 5, 83 (1972); corrected according to a private communication with D. Kleppner as quoted in [21].

[220] W. D. Phillips, D. Kleppner, and F. G. Walther, private communication (1984), quoted as in [21].

[221] E. A. Moore, Mol. Phys. 97, 375 (1999).

[222] N. C. Pyper, Mol. Phys. 97, 381 (1999);

N. C. Pyper and Z. C. Zhang, Mol. Phys. 97, 391 (1999).

[223] Y. I. Neronov, A. E. Barzakh, and K. Mukhamadiev, Sov. Phys. JETP 42, 950 (1975).

[224] Y. I. Neronov and A. E. Barzakh, Sov. Phys. JETP 45, 871 (1977).

[225] Yu. I. Neronov and A. E. Barzakh, Sov. Phys. JETP 48, 769 (1978).

[226] M. V. Gorshkov, Y. I. Neronov, E. N. Nikolaev, Y. V. Tarbeev, and V. L. Tal'roze, Sov. Phys. Dokl. 34, 362 (1989).

[227] R.S. Van Dyck Jr., P. B. Schwinberg, and H. G. Dehmelt, Phys. Rev. Lett. 59, 26 (1987).

[228] K. Pachucki, U. D. Jentschura, and V. A. Yerokhin, Phys. Rev. Lett. 93, 150401 (2004); 93, 229902 (E) (2005);

K. Pachucki, A. Czarnecki, U. D. Jentschura, and V. A. Yerokhin, Phys. Rev. 72, 022108 (2005).

[229] R. N. Lee, A. I. Milstein, I. S. Terekhov, and S. G. Karshenboim, Phys. Rev. A 71, 052501 (2005).

[230] S. G. Karshenboim, V. G. Ivanov and V. M. Shabaev, JETP 93, 477 (2001); Can. J. Phys. 79, 81 (2001).

[231] T. Beier, I. Lindgren, H. Persson, S. Salomonson, and P. Sunnergren, Phys. Rev. A 62, 032510 (2000);

T. Beier, I. Lindgren, H. Persson, S. Salomonson, and P. Sunnergren, Hyp. Int. 127, 339 (2000).

[232] V. A. Yerokhin, P. Indelicato and V. M. Shabaev, Phys. Rev. Lett. 89, 143001 (2002); Phys. Rev. A69, 052503 (2004).

[233] S. G. Karshenboim, R.N. Lee, A.I. Milstein, Phys. Rev. A (2005) to be published; eprint hep-ph/0506060. 
[234] S. G. Karshenboim and A. I. Milstein, Phys. Lett. B549, 321 (2002).

[235] V. M. Shabaev and V. A. Yerokhin, Phys. Rev. Lett. 88, 091801 (2002).

[236] H. Häffner, T. Beier, N. Hermanspahn, H.-J. Kluge, W. Quint, S. Stahl, J. Verdú, and G. Werth, Phys. Rev. Lett. 85, 5308 (2000);

G. Werth, H. Häffner, N. Hermanspahn, H.-J. Kluge, W. Quint, and J. Verdú, In Ref. [13], p. 204.

[237] D. L. Farnham, R. S. Van Dyck, Jr., and P. B. Schwinberg, Phys. Rev. Lett. 75, 3598 (1995).

[238] T. Beier, H. Häffner, N. Hermanspahn, S. G. Karshenboim, H.-J. Kluge, W. Quint, S. Stahl, J. Verdú, and G. Werth, Phys. Rev. Lett. 88, 011603 (2002).

[239] J. L. Verdú, S. Djekic, T. Valenzuela, H. Häffner, W. Quint, H. J. Kluge, and G. Werth, Can. J. Phys. 80, 1233 (2002);

J. L. Verdú, S. Djekic, S. Stahl, T. Valenzuela, M. Vogel, G. Werth, T. Beier, H. J. Kluge, and W. Quint, Phys. Rev. Lett. 92, 093002 (2004).

[240] G. Werth, private communication.

[241] G. W. F. Drake, Can. J. Phys. 80, 1195 (2002).

[242] K. Pachucki and J. Sapirstein, J. Phys. B: At. Mol. Opt. Phys. 35, 1783 (2002).

[243] M. C. George, L. D. Lombardi, and E. A. Hessels, Phys. Rev. Lett. 87, 173002 (2001).

[244] V. W. Hughes and T. Kinoshita, Rev. Mod. Phys. 71, S133 (1999);

T. Kinoshita, In Ref. [13], p. 157.

[245] A. Wicht, J. M. Hensley, E. Sarajlic, and S. Chu, In Proceedings of the 6th Symposium Frequency Standards and Metrology, ed. by P. Gill (World Sci., 2002) p.193.

[246] S. Rainville, J.K. Thompson, D.E. Pritchard, Can. J. Phys. 80, 1329 (2002).

[247] T. Udem, J. Reichert, R. Holzwarth, and T. W. Hänsch, Phys. Rev. Lett. 82, 3568 (1999).

[248] F. Minardi, G. Bianchini, P. Cancio Pastor, G. Giusfredi, F. S. Pavone, and M. Inguscio, Phys. Rev. Lett. 82, 1112 (1999);

P.C. Pastor, P. De Natale, G. Giusfredi, F.S. Pavone, and M. Inguscio, In Ref. [13], p. 314.

[249] J. Castillega, D. Livingston, A. Sanders, and D. Shiner, Phys. Rev. Lett. 84, 4321 (2000).

[250] J. Wen, Ph.D. thesis, Harvard University, 1996 (unpublished).

[251] W. Frieze, E. A. Hinds, V. W. Hughes, and F. M. J. Pichanick, Phys. Rev. A24, 279 (1981). 
[252] S. G. Karshenboim, J. Phys. B 29, L29 (1996), Sov. Phys. JETP 82, 403 (1996).

[253] T. Matsumoto, M. Chiba, R. Hamatsu, T. Hirose, J. Yang, and J. Yu, Phys. Rev. A 54, 1947 (1996); A56, 1060(E) (1997).

[254] W. Quint, private communication. 